\title{
Performance Measurement of Design Patterns for Service-Oriented Architecture
}

\author{
by
}

\author{
Muhammad Kaleem Khan
}

\begin{abstract}
A thesis submitted to
the Faculty of Graduate Studies and Research

in partial fulfillment of

the requirements for the degree of
\end{abstract}

Master of Applied Science

in

Electrical and Computer Engineering

\begin{abstract}
Ottawa-Carleton Institute for Electrical and Computer Engineering
\end{abstract}

\author{
Department of Systems and Computer Engineering \\ Carleton University \\ Ottawa, Ontario \\ January 2013 \\ (C) Copyright 2013, Muhammad Kaleem Khan
}


Library and Archives

Canada

Published Heritage

Branch

395 Wellington Street

Ottawa ON K1A ON4

Canada
Bibliothèque et

Archives Canada

Direction du

Patrimoine de l'édition

395 , rue Wellington

Ottawa ON K1A ON4

Canada
Your file Votre référence

ISBN: 978-0-494-94264-2

Our file Notre référence

ISBN: $978-0-494-94264-2$
NOTICE:

The author has granted a nonexclusive license allowing Library and Archives Canada to reproduce, publish, archive, preserve, conserve, communicate to the public by telecommunication or on the Internet, loan, distrbute and sell theses worldwide, for commercial or noncommercial purposes, in microform, paper, electronic and/or any other formats.

The author retains copyright ownership and moral rights in this thesis. Neither the thesis nor substantial extracts from it may be printed or otherwise reproduced without the author's permission.
AVIS:

L'auteur a accordé une licence non exclusive permettant à la Bibliothèque et Archives Canada de reproduire, publier, archiver, sauvegarder, conserver, transmettre au public par télécommunication ou par l'Internet, prêter, distribuer et vendre des thèses partout dans le monde, à des fins commerciales ou autres, sur support microforme, papier, électronique et/ou autres formats.

L'auteur conserve la propriété du droit d'auteur et des droits moraux qui protege cette thèse. $\mathrm{Ni}$ la thèse ni des extraits substantiels de celle-ci ne doivent être imprimés ou autrement reproduits sans son autorisation.
In compliance with the Canadian Privacy Act some supporting forms may have been removed from this thesis.

While these forms may be included in the document page count, their removal does not represent any loss of content from the thesis.
Conformément à la loi canadienne sur la protection de la vie privée, quelques formulaires secondaires ont été enlevés de cette thèse.

Bien que ces formulaires aient inclus dans la pagination, il n'y aura aucun contenu manquant. 


\section{ABSTRACT}

Service-Oriented Architecture (SOA) is a computing paradigm where large complex applications are composed of independent components offering services to one another through well-defined interfaces. SOA patterns provide generic solutions for different architectural, design and implementation problems of SOA applications. SOA patterns differ in scope and objectives, so their effect on system performance is also different, either positive or negative. SOA patterns introduce means of reliable messaging. Application of patterns facilitates workflow implementations ensuring business processes are completed once started despite system failures. Platform vendors provide native implementation to some patterns hidden from developers. Such enhancements to web service systems compromise system performance. This thesis aims to measures performance effects of applying different patterns at various levels, helping SOA designers to better understand performance impacts of their applications and provide knowledge to make trade-offs between performance and other software properties affected by patterns. A predictive evaluation approach will demonstrate estimating performance early in the lifecycle. 


\section{Table of Contents}

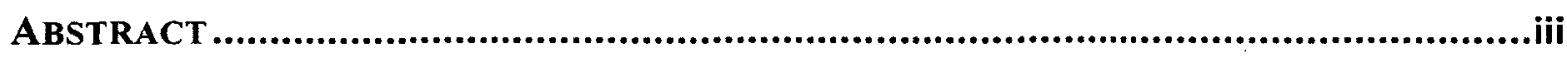

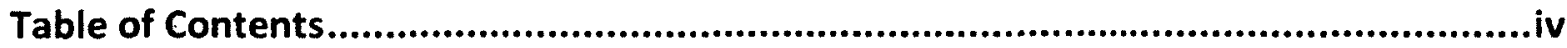

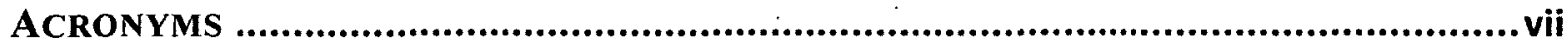

List of Figures ........................................................................................ viii

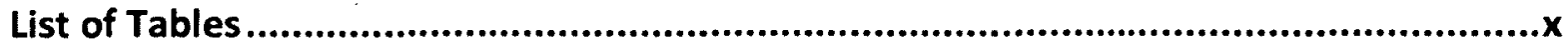

1 INTRODUCTION.................................................................................... 1

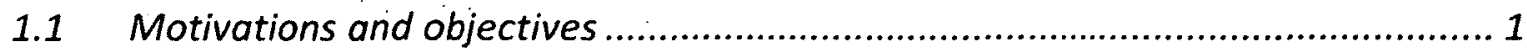

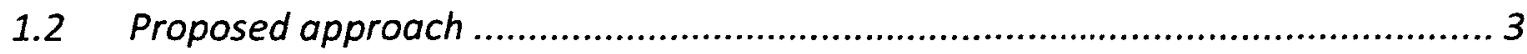

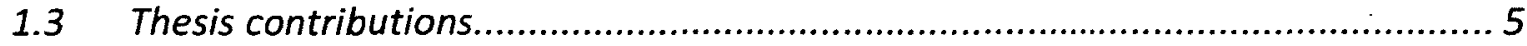

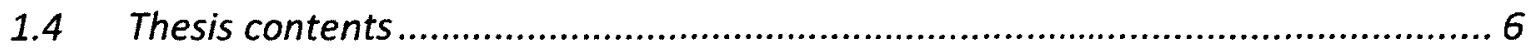

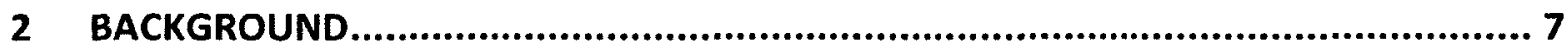

2.1 SERVICE ORIENTED ARCHITECTURE _..................................................... 7

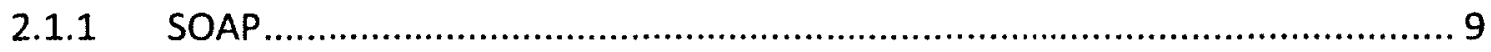

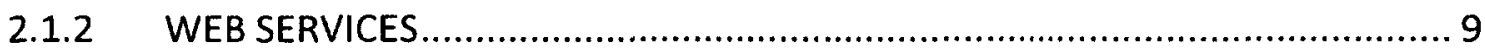

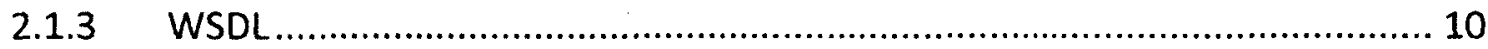

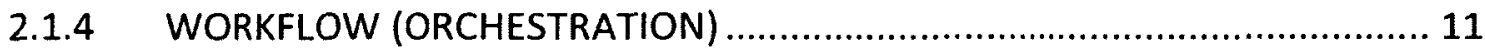

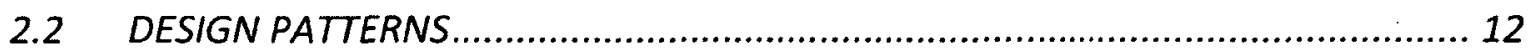

2.3 SOFTWARE PERFORMANCE ENGINEERING ......................................... 14

2.4 TOOLS FOR SOA DEVELOPMENT .......................................................... 16

2.4.1 Microsoft Biztalk server 2010 ......................................................... 16

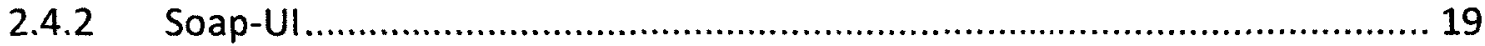

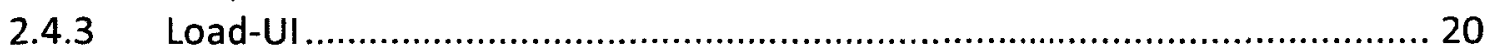

2.4.4 Biztalk Orchestration Profiler........................................................ 20

3 PERFORMANCE MEASUREMENTS OF PLATFORM-PROVIDED PATTERNS ............21

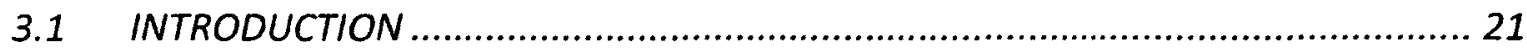

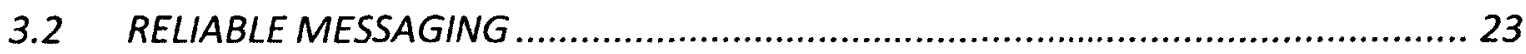

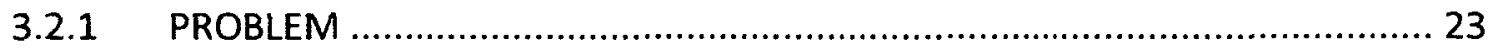

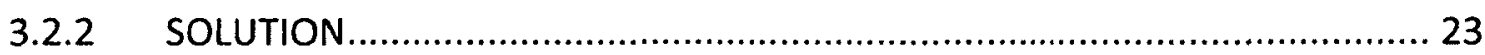

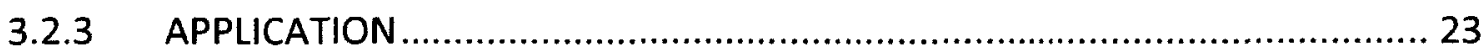

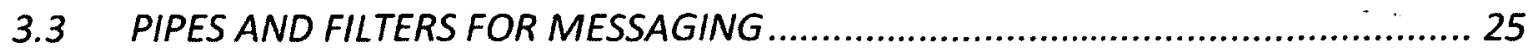

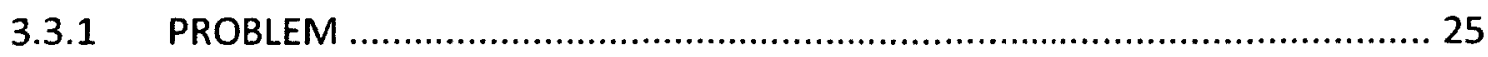

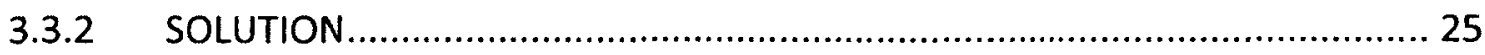

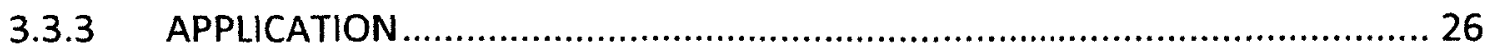

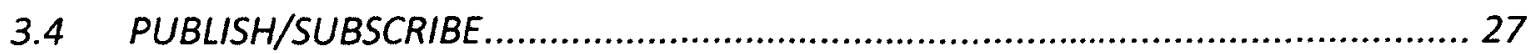

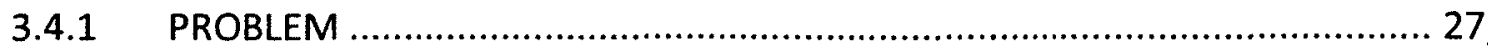

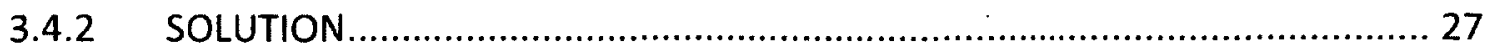




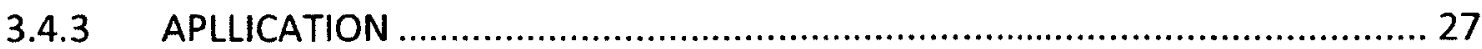

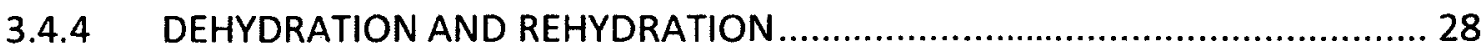

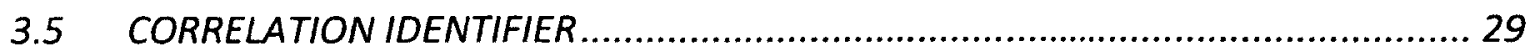

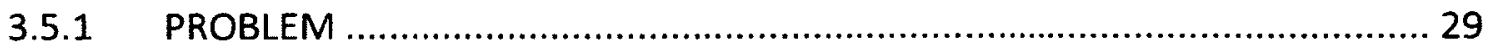

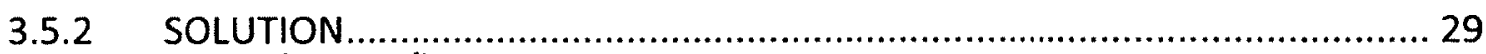

3.6 PERFORMANCE IMPACT OF PLATFORM-PROVIDED PATTERNS................... 31

3.6.1 INVOCATION OF A WORKFLOW SERVICE ....................................... 32

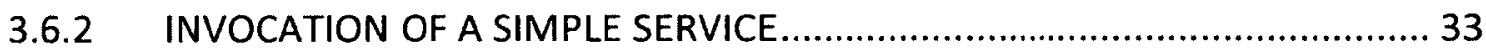

3.6.3 PERFORMANCE OVERHEADS OF WORKFLOW SERVICE AND SIMPLE

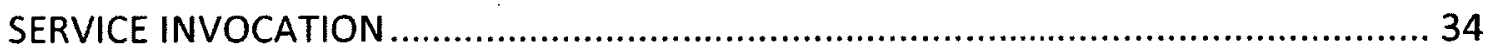

3.7 PERFORMANCE IMPACT OF MESSAGE SIZE .......................................... 37

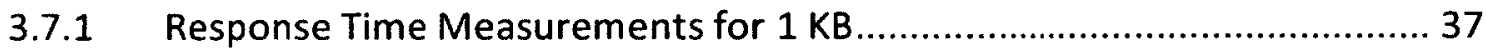

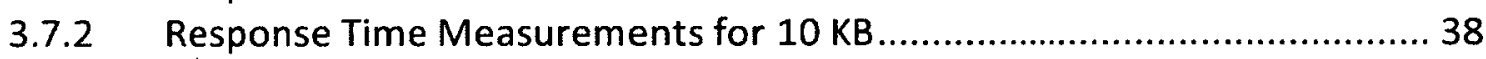

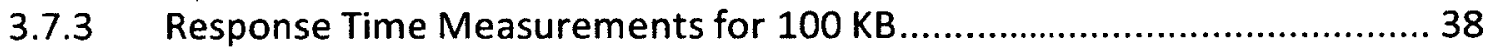

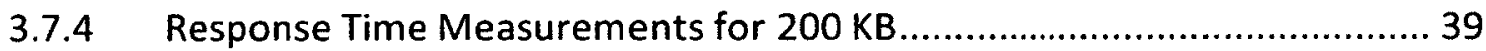

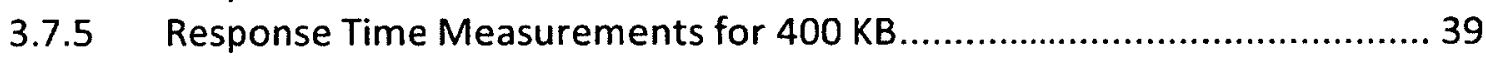

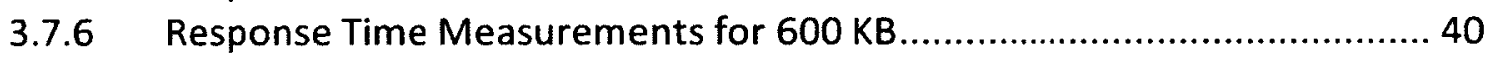

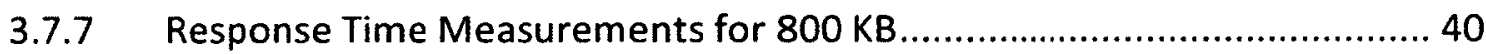

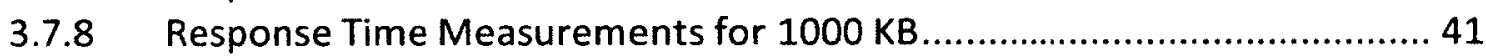

4 PERFORMANCE MEASUREMENTS OF APPLICATION-LEVEL PATTERNS................43

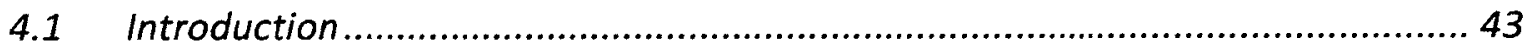

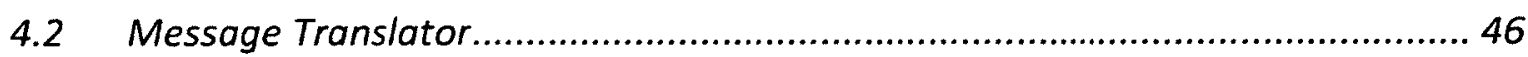

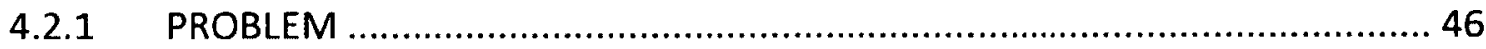

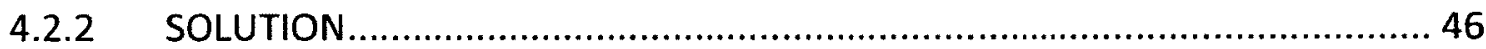

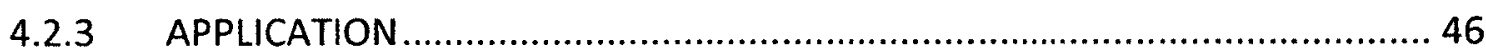

4.2.4 MESSAGE TRANSLATOR PERFORMANCE MEASUREMENTS $\ldots \ldots \ldots \ldots \ldots \ldots . \ldots \ldots$

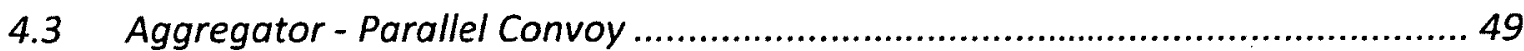

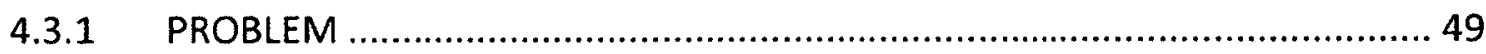

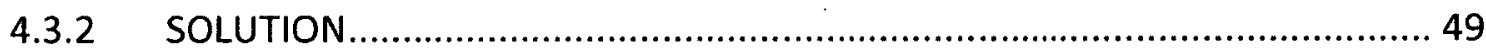

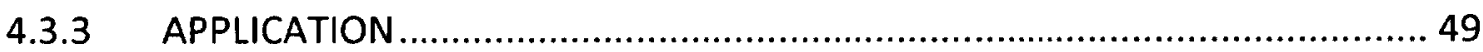

4.3.4 PARALLEL CONVOY MEASUREMENTS ................................................ 52

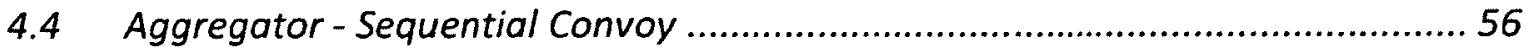

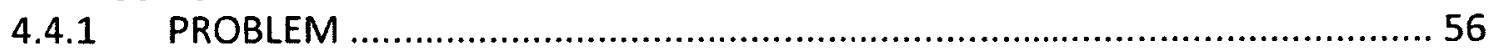

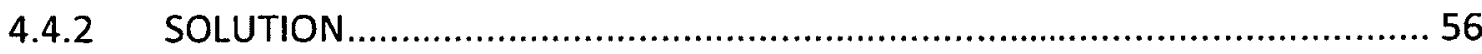

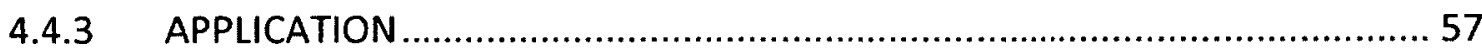

4.4.4 SEQUENTIAL CONVOY PERFORMANCE MEASUREMENTS ....................... 58

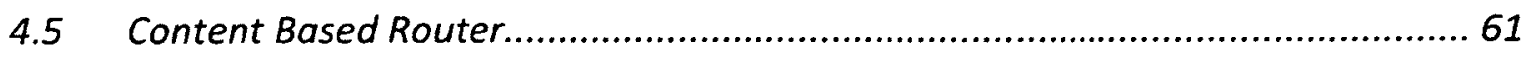

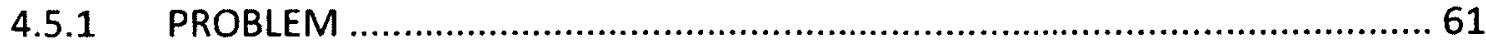

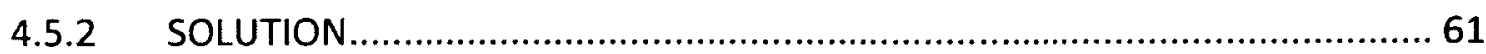

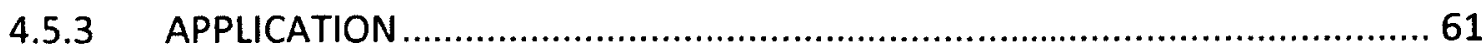

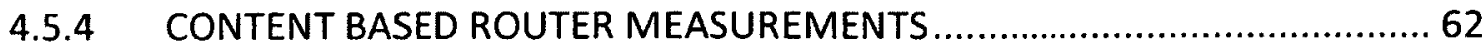

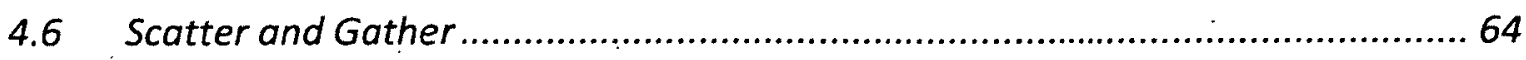

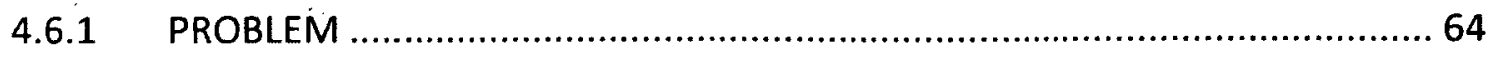




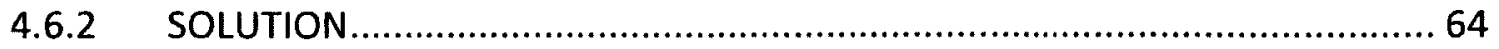

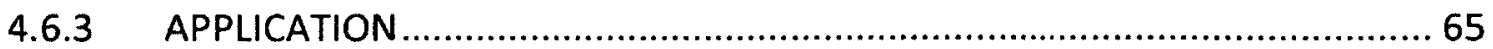

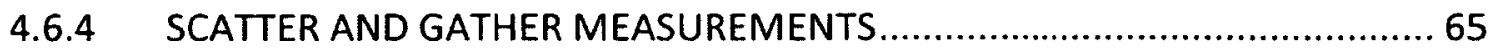

4.7 Orchestration Instance Creation AND restoration after long wait ...................66 66

4.8 RESPONSE TIME MEASUREMENT CHALLENGES ..................................... 68

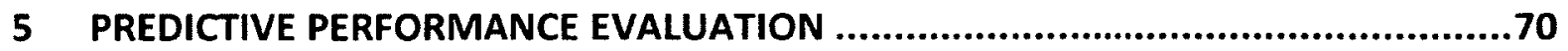

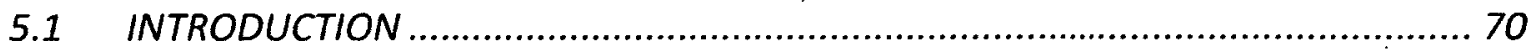

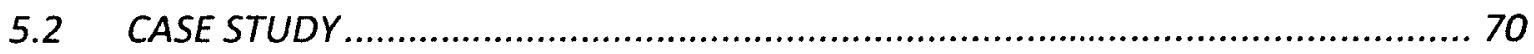

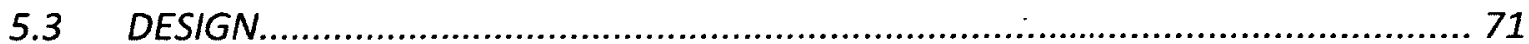

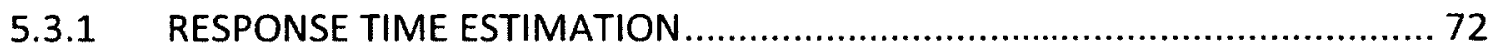

5.4 SYSTEM IMPLEMENTATION IN BIZTALK ............................................. 75

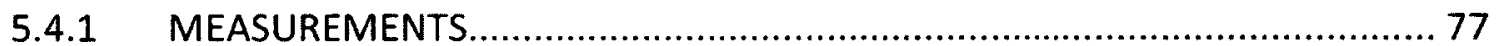

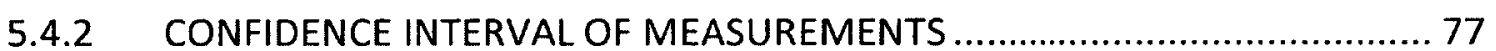

5.5 ESTIMATION WITH VARIABLE MESSAGE SIZE .................................... 78

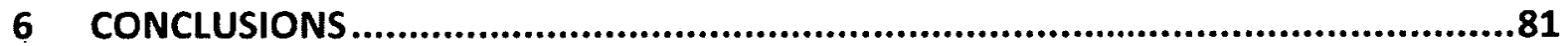

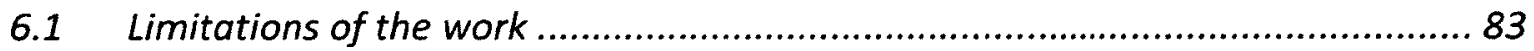

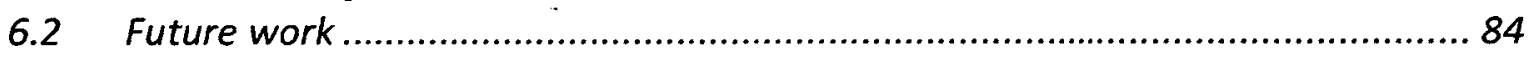

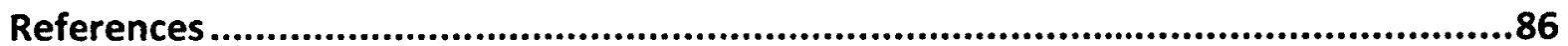




\section{ACRONYMS}

API - Application Programming Interface

BRE - Business Rules Engine

DB - Database

EMR - Electronic Medical Records

XML - Extensible Markup Language

FIFO - First In First Out

HTTP - Hyper Text Transfer Protocol

IIS - Internet Information Services

MSMQ - Microsoft Messaging Queue

REST - Representational State Transfer

SLA - Service Level Agreement

SOA - Service-Oriented Architecture

SOAP - Simple Object Access Protocol

URL - Uniform Resource Locator URL

WCF - Windows Communication Foundation

WSDL - Web Services Description Language

XSD - XML Schema Definition 


\section{List of Figures}

Figure 1. An example of test in soap-UI................................................................ 19

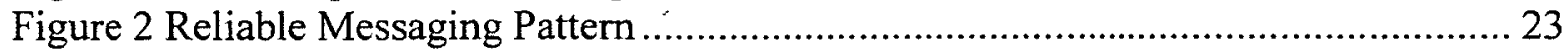

Figure 3 Native reliable message implementation provided by Biztalk .......................... 24

Figure 4 Biztalk WCF Adapter configuration of SOA .................................................. 25

Figure 5 a) Pipes and Filter Invocation b) Biztalk Default Pipeline Stages .................... 26

Figure 6 Creation of a Subscription in Biztalk .............................................................. 28

Figure 7 Correlation Identifier Process................................................................... 30

Figure 8 (a) Correlation attributes. (b) Correlation properties in Biztalk ....................... 31

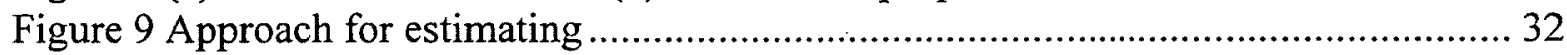

Figure 10 Empty workflow service implemented in Biztalk ............................................ 33

Figure $11 \mathrm{~A}$ simple SOAP based web service implementation ........................................ 34

Figure 12 Response Time Measurement of an empty workflow service invocation......... 34

Figure 13 Response Time Measurements of a simple SOAP service invocation.............. 35

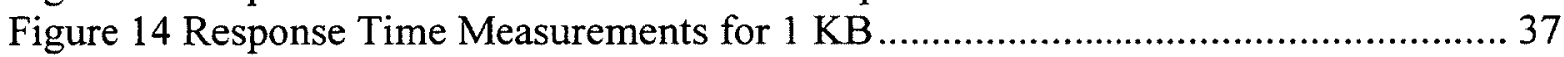

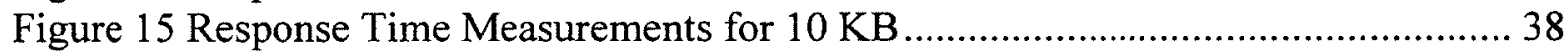

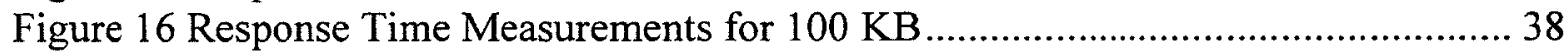

Figure 17 Response Time Measurements for $200 \mathrm{~KB}$................................................. 39

Figure 18 Response Time Measurements for $400 \mathrm{~KB}$.................................................... 39

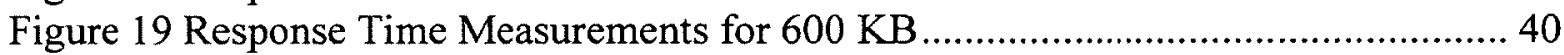

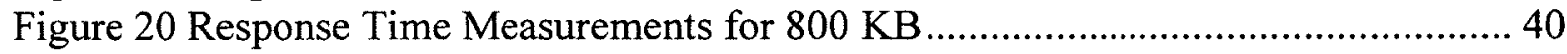

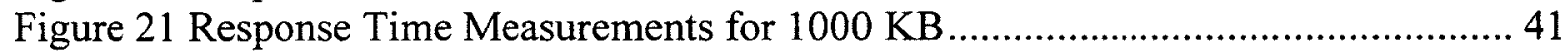

Figure 22 Response Time Versus message size [KB] .............................................. 41

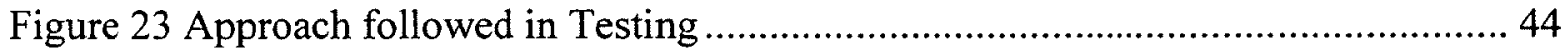

Figure 24 Message Translator Pattern …………........................................................ 47

Figure 25 Message Translator implemented in Biztalk ................................................... 47

Figure 26 (a) Patient Data Representation in Emergency Client (b) Logically equivalent but simplified representation ............................................................................ 48

Figure 27 Message Translator Implementation Response Times .................................... 48

Figure 28 Parallel Convoy Pattern............................................................................ 50

Figure 29 Parallel Convoy Pattern implementation in Biztalk ........................................ 50

Figure 30 Parallel Convoy application in Biztalk to use case ........................................ 52

Figure 31 Parallel Convoy Application Response Times ............................................ 53

Figure 32 Sequential Convoy Pattern ....................................................................... 56

Figure 33 Sequential Convoy pattern application in Biztalk ........................................ 57

Figure 34 Sequential Convoy application in Biztalk to use case...................................... 58

Figure 35 Sequential Convoy Implementation Response Times ..................................... 59

Figure 36 Content Based Router Pattern ...................................................................... 61

Figure 37 Biztalk Implementation of Content Based Router.......................................... 62

Figure 38 Content Based Router Implementation Response Times ................................ 62

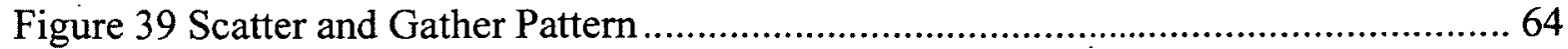

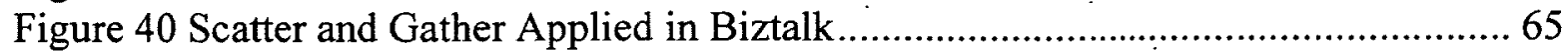

Figure 41 Scatter and Gather Implementation Response Times..................................... 65 
Figure 42 Prescription renewal workflow design (main workflow and insurance service)

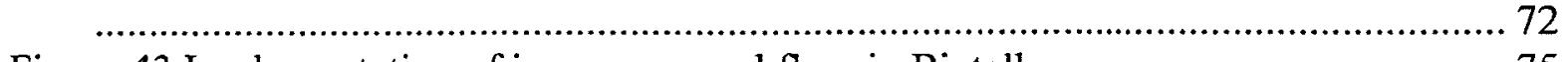

Figure 43 Implementation of insurance workflow in Biztalk ................................... 75

Figure 44 Designer implementation of prescription re-fill workflow in Biztalk............ 76

Figure 45 Patient prescription re-fill request workflow response times ....................... 77

Figure 46 Response time for prescription refill workflow (with $800 \mathrm{~KB}$ messages) ...... 79

Figure 47 Response time for prescription refill workflow (with $400 \mathrm{~KB}$ messages) ...... 79 


\section{List of Tables}

Table 1 Patterns Application to Case Study ........................................................ 22

Table 2 Comparing Average Response time for workflow and ................................. 35

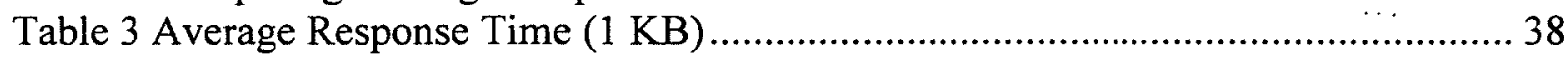

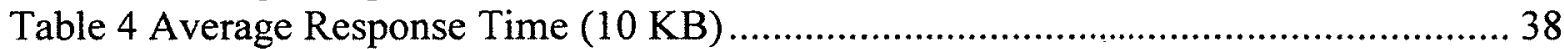

Table 5 Average Response Time $(100 \mathrm{~KB})$.............................................................. 38

Table 6 Average Response Time $(200 \mathrm{~KB})$.............................................................. 39

Table 7 Average Response Time $(400 \mathrm{~KB})$........................................................... 39

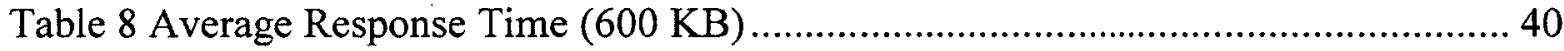

Table 9 Average Response Time $(800 \mathrm{~KB})$........................................................... 40

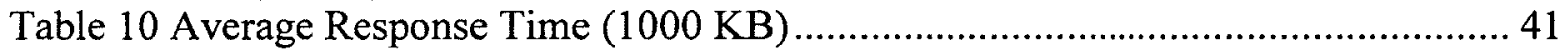

Table 11 Development Patterns Application to Case Study ......................................... 45

Table 12 Average Response Time - Message Translator........................................ 48

Table 13 Average Response Time - Parallel Convoy application for use case............... 53

Table 14 Detailed Assessment at each level of Parallel Convoy ................................ 54

Table 15 Maximum delay associated with Parallel Convoy Receive parts .................... 54

Table 16 Average Response Time - Sequential Convoy application to use case ........... 59

Table 17 Detailed Assessment at each level of Sequential Convoy ............................ 59

Table 18 Average Response Time - Content Based Router ........................................ 63

Table 19 Detailed Assessment at each level of Content Based Router ........................ 63

Table 20 Average Response Time - Scatter and Gather ..........................................66

Table 21 Detailed Assessment at each level of Scatter and Gather.............................. 66

Table 22 Patient prescription refill workflow response time estimates ........................ 74

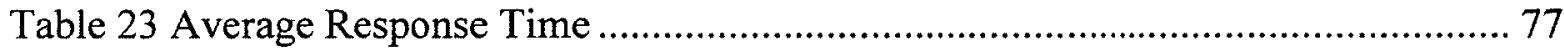

Table 24 Average response time comparison with estimated values ( $800 \mathrm{~KB})$............. 79

Table 25 Average response time comparison with estimated values $(400 \mathrm{~KB}) \ldots \ldots \ldots \ldots . . . .80$ 


\section{INTRODUCTION}

\subsection{MOTIVATIONS AND OBJECTIVES}

Service-oriented architectures (SOA) is a distributed computing paradigm where large complex applications are composed of independent software components running on heterogeneous platforms that offer services to one another through well-defined interfaces. According to (Earl, 2005), SOA and the supporting technologies have matured to the extent that proven design practices have been distilled in the form of SOA patterns, which provide generic solutions for different architectural, design and implementation problems. After years of research, reviews and validation, this body of work has been documented as a comprehensive collection of over 90 generic SOA design patterns (SOA-Patterns, 2012). Other publications express SOA patterns in terms that are specific to a certain technology (Rostova, 2011) or give implementation examples in different technologies (Hohpe and Woolf, 2003). There are different supporting technologies (also known as service platforms) that support the implementation of SOA systems, each providing different features for publishing, discovering and invoking services. Two wellknown SOA platforms are web services based on standard such as SOAP, Extensible Markup Language (XML) and Web Services Description Language (WSDL) (Earl, 2005) and Representational State Transfer (REST) services using HTTP verbs such as GET, POST, PUT, etc. (Fielding, 2000). A side effect of using a service platform is the performance overhead introduced by the platform operations; these overheads impact the overall performance of a SOA application and are different from platform to platform.

The scope of different SOA patterns varies widely, from one single service, to a set of services, to the extent of the entire enterprise architecture. The objectives of the SOA 
design patterns are also very varied, each attempting to improve a certain functional or non-functional property. Consequently, the effect of the design patterns on the system performance is also very different: some have a positive effect while others a negative one (which usually represents the price to pay by doing more work in order to improve some other properties, such as maintainability, security, dependability, etc.).

Performance characteristics (such as response time and throughput) are essential quality attributes of every software system, especially when humans are interacting with the system and are waiting for replies or when the system needs to react to external events in real-time. Many performance problems are due to fundamental architecture and design factors rather than to inefficient coding (Smith and William, 2002). In order to assess performance risks, quantitative performance assessments are necessary in early design phases, when the system is not implemented yet and cannot be measured. For this reason, the Software Performance Engineering (SPE) techniques proposed in the late 80's have been increasingly accepted and practiced (Smith, 1990), (Smith and William, 2002). SPE proposes to use quantitative methods and performance models, such as Queueing Networks (Lazowska et al., 1984), Layered Queueing Networks (Woodside et al, 1995) or Petri Nets (Ajmone Marsan et al., 1995) to assess the performance effects of different design and implementation choices through the development of a system. SPE supports the idea that the integration of performance analysis into the software development process, from the initial stages to the end, can insure that the system will meet its performance objectives. This would eliminate the need for "late-fixing" of performance problems, a frequent practiced approach that postpones any performance concerns until 
the system is completely implemented. Late fixes tend to be very expensive and inefficient, and the product may never reach its original performance requirements.

Since SPE proposes to use predictive performance models, the models need to indicate the quantitative resource demands made by different parts of the software. Example of resource demands are CPU execution time, number of visits to disk, number of invocations of a service, etc. In general, it is difficult to estimate these numbers in the early design phases. Besides the resource demands made by the application software itself, the service platforms have also a performance cost, which needs to be captured in the performance model. In order to build a performance model, the analyst needs to know what resources are required and how much they are used by the application and by the underlying platforms. It is important for application designers to understand the performance overheads introduced by different service invocation mechanisms.

The objective of the thesis is to investigate the performance effects of different design patterns through performance measurements and to get an idea of how long it takes to execute them. The results will help SOA designers to better understand the performance consequences of applying different patterns, and will give them insight and knowledge for making better design decisions.

\subsection{PROPOSED APPROACH}

There are various tools and platforms with similar capabilities to implement SOA and workflow solutions. Typically a tool provides the capability to design and host a business process as well as integration with third party systems leveraging both proprietary and standard protocols, such as SOAP based web services. In our research we have utilized Microsoft Biztalk server to design and host business processes (BizTalk, 2010) mainly 
because it is well known and also available. Biztalk Developer Edition was free, with almost all the features offered by the Professional Edition. We used WCF (Windows Communication Foundation) to expose the business processes created in Biztalk as SOAP-based web services which will offer a WSDL interface to the client. We also used SOAP-UI, which is a web service testing tool. It allows creating test cases to invoke web services by generating a web service proxy on the client side. Also we utilized the LOAD-UI tool to automate the execution of test cases created in SOAP-UI, as well as to obtain statistics such as average response time of web service invocations.

We conducted our measurements of pattern applications around a case study in the health care domain to handle and transfer patient data in emergency situations. This case study has various use cases which we realized by applying different SOA design patterns, mostly targeted to messaging and to service invocation. The implementation of some patterns related to the invocation of services and messaging is provided natively by Biztalk and is hidden from the user. We estimated the invocation overhead which includes the hidden patterns by calling an empty business process which does nothing, so its entire response time is due to the actual invocation. We built our system by incrementally adding use cases of the case study, utilizing various patterns and measuring the response time. Due to the fact that the response time is a stochastic variable, the measurements of each experiment was performed repeatedly (usually around 180 times) to estimate the variance and to achieve an acceptable level of confidence in the results. The repeated requests were spread over one hour interval, leading to a frequency of 3 requests per minute, which allowed the system to complete a request before subsequent test invocations, and thus to avoid the waiting of a request for previous ones. This was 
done to insure that the resource usage was due to one request at a time. During measurements, all other running processes were terminated (with the exception of operating system processes over which the user has no control) in order to obtain consistent measurements.

\subsection{THESIS CONTRIBUTIONS}

This research will allow getting better insight into performance patterns utilized in SOA and overhead occurred in various stages of service-based workflow invocations.

Part of the outcome of this research has been published in the following paper:

M. Kaleem Khan, Dorina C. Petriu, "Performance Measurements of Design Patterns for Service-Oriented Architecture", Proc. of the International Conference on Electrical and Computer Engineering ICECS' 2012, Ottawa ON, Canada, August 2012.

The thesis contributions are as follows:

- Measure the performance effects of SOA design patterns related to messaging and service invocations, and study their dependency on the message size. For these measurements, the design patterns were classified in two categories: patterns implemented by the service platform (in our case the Biztalk server), which are hidden from the developer, and patterns explicitly applied by the developer at the application level.

- Predictive estimation of the response time for a new system in the design phase, before it was implemented based on the patterns measurements results obtained previously. After that, the system was implemented and measured, which allowed us to verify the early predictions against the measurements of the real 
implementation. This prediction approach gives the designer early insight into the execution times for different service platform operations that include hidden patterns, as well as application level patterns, allowing the developer to make informed design decisions in early development phases.

\subsection{THESIS CONTENTS}

The thesis consists of six chapters.

Chapter two presents the background on concepts, such as SOA, Design patterns, Software performance engineering, as well as on service platform (Biztalk Host), development tools (Biztalk Orchestrations), and testing tools (SOAP-UI, LOAD-UI).

Chapter three investigates what design patterns are natively provided by platform, what is their role and measures their cumulative overhead.

Chapter four presents the application development level SOA patterns by the application of a case study in the health care domain handling patient data transfer in emergency situations.

Chapter five presents the predictive estimation of end-to-end response time for a SOA application in its early design phase. Then the application is implemented and measured, in order to compare the estimated values against the actual measured performance.

Chapter six presents the conclusions and directions for future work. 


\section{BACKGROUND}

In this chapter, we describe background knowledge regarding concepts, architecture, tools and frameworks utilized in our research and development process. The following issues will be discussed:

- SOA - Service Oriented Architecture

- SOAP based Web Services, WSDL.

- Business Processes or workflows. (In Biztalk server, these are also known as Orchestrations).

- Exposing Business Process as SOAP based web services.

- Difference between simple SOAP based service and Orchestrations as SOAP based service.

- Design Patterns, including SOA patterns

- Software Performance Engineering

- Tools for service-oriented development: Microsoft Biztalk Server.

- Tools for testing and statistics: soapUI, loadUI, Biztalk Orchestration Profiler.

\subsection{SERVICE ORIENTED ARCHITECTURE}

Service-oriented architectures (SOA) is a distributed computing paradigm where large complex applications are composed of independent software components running on heterogeneous platforms that offer services to one another through well-defined interfaces(Earl, 2005). These services are well-defined business functionalities that are built as software components that can be reused for different purposes. There are different supporting technologies (provided by the service platforms) that support the implementation systems, each providing different features for publishing, discovering 
and invoking services. Two well-known service platforms are web services based on standard such as SOAP, XML and WSDL (Earl, 2005) and Representational State Transfer (REST) services using HTTP verbs such as GET, POST, PUT, etc. (Fielding, 2000). In this work, we use web services based on SOAP, XML and WSDL, which have undergone an important standardization process.

SOA is not directly related to any technology, although it is most often implemented with web services, which are very appropriate for SOA realization. However, just using web services is not adequate to build SOA. We have to use web services according to the design concepts that SOA defines (Earl, 2005). The service approach is ideally suited to more loosely coupled systems. Some of the advantages of using SOA are:

- Modularity: As appropriate services are dynamically coupled, it is relatively easy to integrate new services into the framework.

- Interoperability: Due to standardization of the communication and description of the services, third party services can easily be incorporated as required.

- Extensibility: Due to the relative ease with which services can be incorporated into a system, there is less danger of technology 'lock-in'.

- High Autonomy: Services will likely be developed by distributed autonomous teams.

- Coarse Granularity: Services will tend to be more coarse-grained than traditional components, and there is a need to reconcile many aspects of a complex activity.

- Process Awareness: Services will be driven by explicit business process, which means there is a close relation to the domain workflows. 


\subsubsection{SOAP}

Simple Object Access Protocol (SOAP) is a protocol for sending messages across heterogeneous distributed systems. SOAP is based on XML. It is relatively easy for humans to read and understand the structure of a SOAP message. SOAP messages are always wrapped in a container called the envelope; the envelope always contains a body, which carries the payload of the message in an XML documents. The envelope includes headers that can optionally contain information like transaction context, security information. If something goes wrong during processing, a SOAP fault will be added as the body content and sent back to client (Hewitt, 2009).

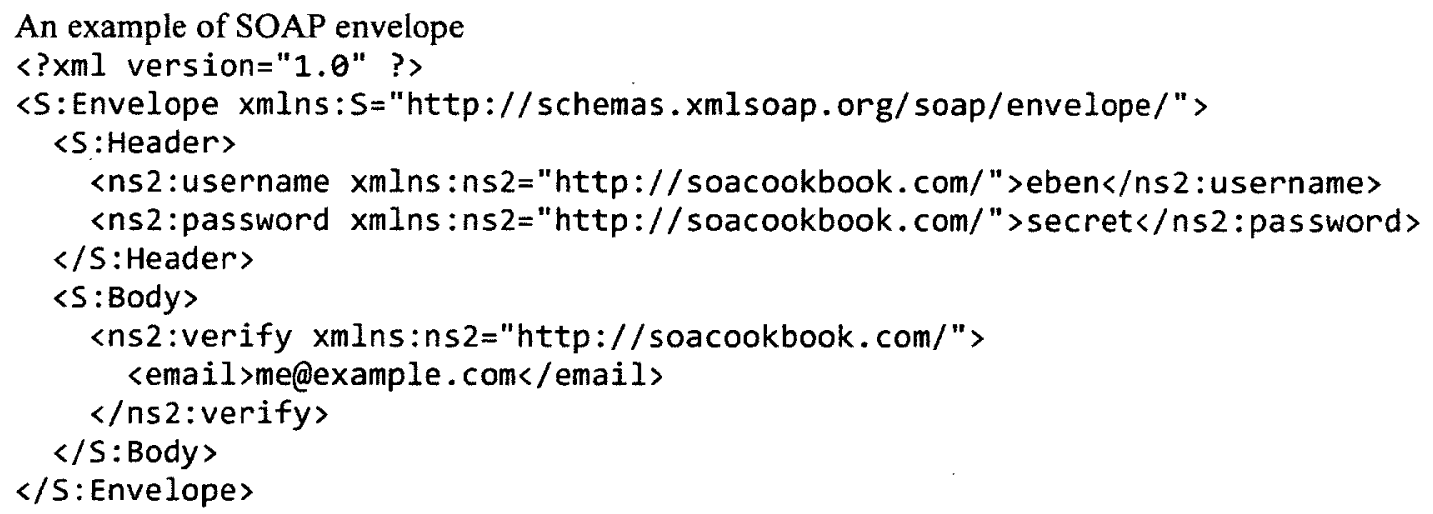

\subsubsection{WEB SERVICES}

Web services are a commonly used technology for interoperability and integration of applications and information systems. They are a suitable technology often used for the implementation of SOA. Web services provide the foundation for achieving interoperability between applications using different software platforms, operating systems, and programming languages. They are built on XML most commonly with SOAP or Representational State Transfer (REST) protocols. A Web Service can provide one or more operations, which look like an ordinary function call to the client. The client 
generates a proxy method to call web services and these calls are translated into a SOAP envelope and transmitted across the wire to the server or service provider. Operations in web services are based on the exchange of XML-formatted payloads. They are a collection of input, output, and fault messages. The combination of messages defines the type of operation (one-way, request/response, solicit response, or notification).

\subsubsection{WSDL}

WSDL is the acronym for Web Service Description Language, which was defined as a way to describing a web service in a platform independent manner. The client uses the WSDL definition to generate a proxy, which is then invoked like an ordinary method call.

public String verify( @WebParam(mode=WebParam.Mode.IN, name="email")String email,

@.WebParam(mode=WebParam.Mode.INOUT, header-true, name="username") Holder $<$ String $>$ username,

@ WebParam (mode=WebParam.Mode.INOUT, header=true, name="password") Holder $<$ String $>$ password)

\{ / Do Something \}

The WSDL for the above JAVA implementation is expressed as

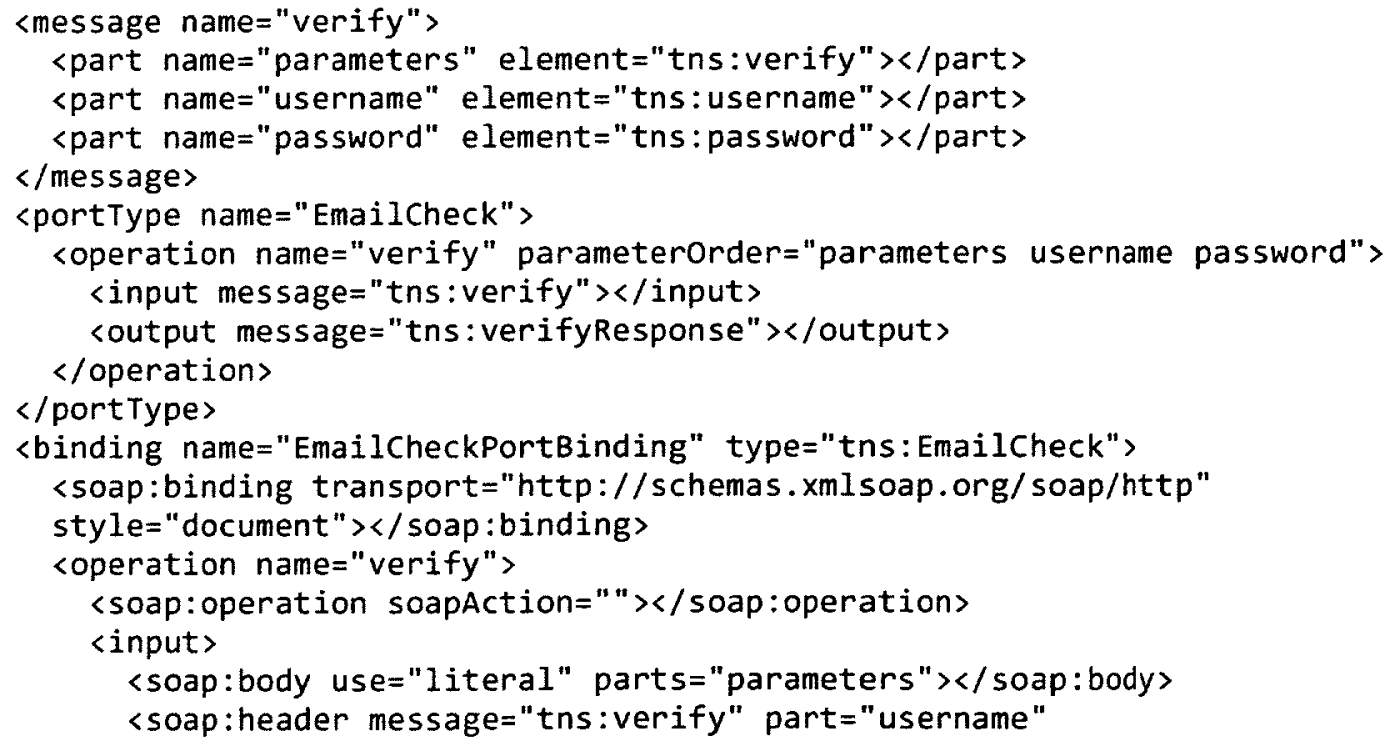




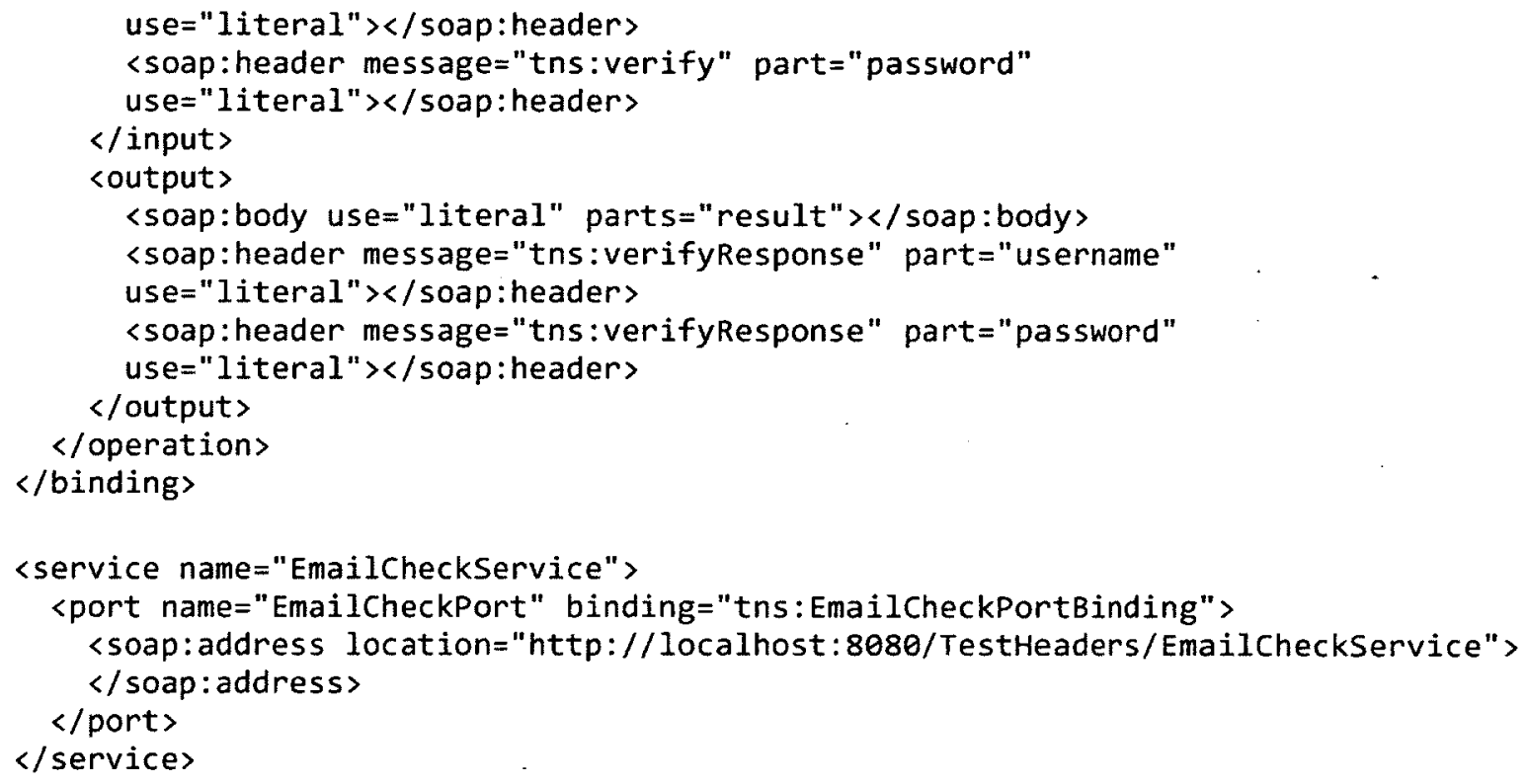

\subsubsection{WORKFLOW (ORCHESTRATION)}

Workflow represents a sequence of connected steps that represent an abstraction of real work, and are used to design and develop long running business processes. There are many model-driven workflow development tools providing the ability to design workflows graphically, which are easier to design, maintain or make changes than writing code. For example, a workflow could be receiving Order-1 from customer-1, then in the next step confirming their payment information, finding the customer address and finally sending a message to warehouse for actual shipment. Workflow can also contain alternate paths, such as if payment cannot be verified, then inform the customer.

Workflow services are the result of exposing a workflow as a Web Service, which to the client looks like an ordinary web service described by its WSDL, as mentioned in the previous section. Behind the scene, workflows have lot more functionality than a plain web service, but this also affects the performance of the system. Workflows are hosted in an environment such as the Biztalk server discussed in section 2.4.1. The hosting 
environment offers many feature like message persistence and routing through end points as discussed in section 2.4.1.

\subsection{DESIGN PATTERNS}

A design pattern explains a particular design problem that arises in a specific design context and gives a well-proven solution for the problem. The first book of design patterns (Gamma et al., 1995), documented the structure and participants in common object-oriented program microstructures. A pattern language is a family of interconnected patterns that define a process for resolving software development problems systematically.

Design patterns can be seen as a means of transferring design knowledge. They represent an efficient way of studying and later on reusing the designs of experienced software engineers. In contrast to a methodology that tells us how to do something, a design pattern shows us how to do it. Object-oriented design patterns are intended to alleviate costly rediscovery and reinvention of core software concepts and abstractions. Patterns provide a way to encapsulate the design knowledge that offers solution to standard software development problems. However, patterns could only enable reuse of abstract design and architecture knowledge, and do not directly provide reusable code.

The research field of design patterns is very fertile. The scale of design pattern varies from architectural level, to mid-level design to language idioms (Buschmann et al, 1995), (Schmidt et al., 2000). Design patterns have been investigated for different domains, such as security and service-oriented architecture.

Different security patterns and security pattern systems are presented in (Schumacher, Roedig, 2001) and (Yoder and Barcalow, 1997), where a security pattern describes a 
certain recurring security problem and presents a well-proven generic scheme for its solution, while a security pattern system is a collection of security patterns that are implemented and combined for practical use.

In the SOA world various patters are defined which are meant to improve both nonfunctional and functional qualities of the system. Different authors use a variety of notations and vocabulary to describe the patterns. For instance, (Earl, 2009) defines various categories of patterns which are very general and valid for any SOA systems, regardless of the technology that supports the implementations, whereas there are patterns that focus on a specific technology and are more specific.

Moving one step further, the enterprise application integration patterns (Hohpe and Woolf, 2003) mainly expand on Service Messaging, Transformation and Common Compound Design categories of the patterns from (Earl, 2009). These patterns provide generic solutions when creating documents schemas, perform message translation, message routing to specific or multiple parties and sequencing of various steps to form a workflow solution as well ways to implement communication mechanisms such as synchronous/asynchronous communications and message queuing.

Various platform vendors provide native implementation of some patterns, using their proprietary vocabulary. Further they provide guidelines to developers in form of patterns that can be leveraged at design time, such as sequential and parallel convoys. Many of the patterns at finer granularity are implemented as part of the platform and offer native Application Programming Interface (API) implementations. For example, BizTalk server creates many different databases, such as MessageBox and Subscription, when it is 
installed, which support the realization of many patterns described in (Erl, 2009) as platform-provided patterns.

\subsection{SOFTWARE PERFORMANCE ENGINEERING}

Software Performance Engineering (SPE) is a systematic, quantitative approach to construct software systems to meet their performance objectives proposed by Connie Smith (Smith, 1990). SPE uses quantitative performance models to identify architectural, design, and implementation alternatives that will meet performance objectives (Smith, 1990), (Smith and William, 2002). SPE is a model-based and software-oriented approach. Modeling is central to both SPE and object-oriented development. By building and analyzing performance models of the proposed software, we can discover its characteristics to decide if it will meet its requirements before we actually commit to build it. Different kinds of performance models can be used, such as Queueing Networks (Lazowska et al.,1984), Layered Queueing Networks (Woodside et al, 1995), (Alhaj and Petriu, 2010) (Mani et al, 2012) or Petri Nets (Ajmone Marsan et al., 1995). In SPE, performance study begins as early as possible in the development process, when a preliminary design exists.

It is believed that integration of software performance engineering into the software development process will greatly improve the software quality and lower the risk of software failure. The type of performance models used depends on the purpose of analysis, the level of detail available, and the precision of the input data. Early in the software development process, the knowledge of the software's design and implementation details is insufficient to model the system's performance precisely. Simple models with estimated parameters are used to get rapid feedback on whether the 
proposed software is likely to meet the performance objectives. Rough performance estimate at an early stage can prevent costly performance mistakes that may make the systems miss its performance requirements by orders of magnitude. As software development progresses, more and more details of the software design and implementation become available, so it's possible to replace the rough estimates of resource usage with more detailed estimates or with measurements of actual usage. Consequently, the accuracy of the performance predictions can improve while the project progresses.

Software architectures need to be decided at the earliest stage in a software development project. Architecture is a specification of the components of a system and the interaction between them. Architecture guarantees certain behavioral properties of a conforming system and can be a powerful tool to aid the process of predicting the behavior of a system with that architecture, of managing the construction of a system, and of maintaining it.

The architectural decisions have the greatest impact on software quality. Although a good architecture alone could not guarantee that the product will reach the quality goals, a poor architecture surely can prevent their realizations. Inappropriate decisions or errors at early stages will cost excessively or cause the failure of the project. Most performance failures are because of a lack of consideration of performance issues early in the development process and in the architectural design stage. The "fix-it-later" approach is dangerous (Smith, 1990); it could not save the project if its major architecture is not suitable to handle the required tasks, no matter how the software is implemented. 


\subsection{TOOLS FOR SOA DEVELOPMENT}

\subsubsection{Microsoft Biztalk server 2010}

Microsoft BizTalk Server (BizTalk, 2010) provides a development and execution environment that provides the ability to design and host workflow systems that run as long as weeks or months. BizTalk Server features include the ability to define business document specifications and how these documents have to be transformed when passed between applications, and the ability to monitor and log server activity. These documents are the XML based schema that defines structure of an actual document instance.

Biztalk server provides a standard gateway for sending and receiving documents across the network as well as providing a range of services that ensures data integrity, delivery, and security.

BizTalk Server features include the ability to define business document specifications such as XML Schema Definition (XSD) schemas and how these documents have to be transformed when passed between applications (messaging), and the ability to monitor and $\log$ server activity. BizTalk Messaging Services provides the ability to send business documents securely and reliably. BizTalk Orchestration or workflow services enable to define the business processes that are used to create the messages and then implement them using an integrated graphical environment.

BizTalk server provides many flexible developments features mentioned briefly below.

\section{Orchestrations}

The orchestration designer tool provides many shapes (i.e., diagram blocks) such as receive and send to receive and send messages, respectively. It also has a Call Expression shape to call any reusable code written in any language using .Net framework. The 
Decision shape provides to write IF-ELSE logic in a graphical manner. The Construct message and Transform shape together provide the capabilities to create new messages inside the Orchestration.

\section{Send Ports}

BizTalk send ports provide a means of out-bound communication with outside world. The send ports are created to send messages to other web services and other proprietary interfaces such as SAP, Oracle, PeopleSoft etc. The send port implements an adapter to convert BizTalk native messages which are XML to proprietary message format. Behind the scene, a Biztalk send port implements Publish-Subscribe pattern as the out bound messages are written to a MessageBox which is a relational database. There is subscription resolution process that keeps monitoring the MessageBox. As soon as it finds a message matching a particular subscription, it de-queues the message and forwards it to the relevant send port for transmission or invokes a workflow instance if the message is intended for a workflow. The send port also implements a message translator pattern, which translates message from XML to the format expected by the receiving party.

\section{Receive Locations}

Third party systems send messages to BizTalk receive ports which have a URI defined. The receive location can implement an adapter to translate proprietary formats into XML upon reception. The receive location implements the message translator pattern which translates message from the sending party format to XML. Internally, Biztalk processes all messages as $\mathrm{XML}$. The receive location behind the scene also implements reliable 
messaging pattern as it submits all inbound messages to MessageBox, which is implemented as a relational database.

\section{Receive Ports}

Receive ports are logical grouping of receive locations. Biztalk orchestration can receive messages from various sources in different format which have the same logical meaning. Each receive location is created to handle a particular type of incoming message, whereas a logical grouping of receive location into a receive port provides a unified interface to the orchestration.

\section{Web Service Invocation}

BizTalk provides the ability to call $3^{\text {rd }}$ party SOAP based web services. When a BizTalk solution with a reference to SOAP based web service is deployed in a host environment, it creates appropriate receive location and send ports for request-response operations of the web service. A simple web service invocation does not have the overhead of reliable messaging, publish-subscribe, pipe and filters and hydration and rehydration (described in more details in the next chapter) however it does the job of forming a SOAP envelope on the sending end and parsing of a SOAP envelope on the receiving end.

\section{Orchestrations as SOAP Web Services}

BizTalk provides the ability to expose an orchestration or workflow as a web service. To the client, the WSDL appears as a normal web service with request-response operations with a document schema, but when invoked, it starts an Orchestration within the BizTalk environment. Orchestrations are utilized for business applications that require greater degree of reliability involving communication with various business partners, for example an airline reservation company may also offer car rental and hotel booking 
services to its clients which requires use of orchestrations. The implementation of orchestrations as web services requires reliable messaging, publish-subscribe, correlation and hydration and rehydration, all of which are expensive to implement.

\subsubsection{Soap-UI}

soapUI (soap-UI, 2012) is a tool for Testing SOAP based Web Services. soapUI is an

Open Source and completely free tool with a commercial companion. soapUI can be

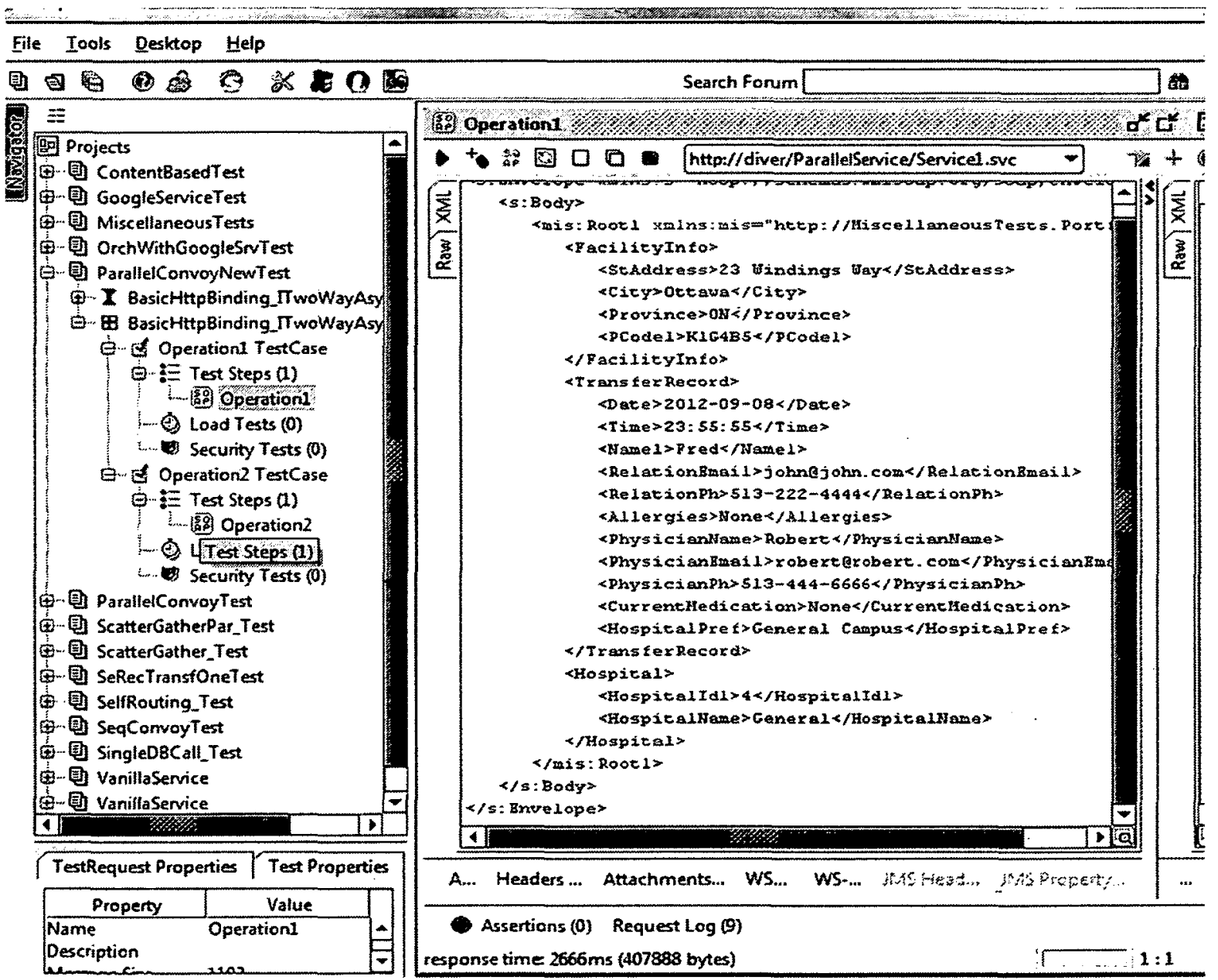

\section{Figure 1. An example of test in soap-UI}

used for functional, performance, interoperability, and regression testing of web services.

soapUI allows importing the WSDL definition of a web service, and then it generates a 
web service proxy and client, and calls the web service. Beside many other functions, it provides traces of SOAP messages exchanged by client and web service, as well as endto-end execution time measurement. It allows for the creation of test cases with assertions, like any other unit testing tool or framework. Although soapUI allows measuring end to end response time from the invocation of a web service until its response is received, it lacks the ability to perform measurements at finer granularity, such as eliminating the time required to form SOAP envelop and only measure the time from invocation of orchestration to its completion. Figure 1 shows a test case as implemented in soapUI.

\subsubsection{Load-UI}

LoadUI (loadUI, 2012) is a web service testing automation tool. It allows importing test cases created in soapUI and then automatically invokes the test cases. Using loadUI, the total testing can be defined as given number of test cases to be executed per second, minute or hour. It has various data logging tools with capabilities to generate graphs and summary reports.

Examples of graphs generated by loadUI are shown in Chapters 3 and 4 .

\subsubsection{Biztalk Orchestration Profiler}

The orchestration profiler is a tool that provides instrumentation for Biztalk orchestration execution. It generates reports over the specified time period and displays the time consumes at each step of an Orchestration. However, it does not capture the time consumed since the arrival of messages at a receive location until the orchestration instance is created. This tool was used in the thesis for performance measurements. 


\section{PERFORMANCE MEASUREMENTS OF PLATFORM- PROVIDED PATTERNS}

\subsection{INTRODUCTION}

For developing service oriented application, there is lot of support and prebuilt functionality provided by the development tools and runtime such as Biztalk server. Many of these features or in some cases frameworks describe a pattern documented in SOA literature. Implementation of these patterns provide infrastructure to develop messaging applications and implementation of these patterns is part of plumbing provided by platform such as Microsoft Biztalk. The developers are shielded away from the complexity of implementing these patters. Because implementation of these patterns is hidden under the platform, any changes to their application or measuring exact performance overhead associated to them in isolation can be challenging. However, during implementation of business processes in our case study, we found that a good estimate of performance penalty can be gauged by eliminating the overhead caused by known components from the end to end response time.

There are other patterns which are applied to problems in a business domain and onus of implementation is on developer using the graphical notation provided by the vendor. The implementation and naming of these patterns may vary from one tool to another. For example, Microsoft documents Sequential and Parallel Convoy patterns to correlate multiple arriving messages inside a given business process instance. We will examine them in more detail in next chapter. 
In this chapter, our focus is on components of Service Based system that are provided natively by Biztalk server and will gauge the accumulative overhead caused by service invocation. We will try to estimate the time required for execution of a component in isolation wherever possible.

The following table lists the pattern applied to use cases of our study:

Table 1 Patterns Application to Case Study

\begin{tabular}{|l|l|}
\hline $\begin{array}{l}\text { Reliable } \\
\text { Messaging }\end{array}$ & $\begin{array}{l}\text { Patient data must be protected against unforeseeable circumstances } \\
\text { causing lost or missing data. }\end{array}$ \\
\hline Pipes and Filters & $\begin{array}{l}\text { Patient data must be transmitted via invocation of SOAP based Web } \\
\text { Service. }\end{array}$ \\
\hline $\begin{array}{l}\text { Publish - } \\
\text { Subscribe }\end{array}$ & $\begin{array}{l}\text { There may be multiple cases in progress at the same time so a } \\
\text { mechanism is required to ensure that data from various patients } \\
\text { belong to distinct patients if arriving from multiple sources }\end{array}$ \\
\hline
\end{tabular}

The tests were performed locally on a single computer. All the simple web services, workflow services and tests were created on deployed on same computer to avoid a highly noisy network delay. Moreover the tests were performed assuming a single user at any given time, so the response time should include only service times, not queueing delays due to contention. A computer with following configuration has been utilized:

- Intel i7, 2.2 GHz CPU

- $6 \mathrm{~GB}$ installed memory

- 64-bit Windows-7 operating system

- Biztalk Server 2010 Developer edition

- soapUI and loadUI

- Biztalk Orchestration Profiler

- Visual Studio 2010 and .Net framework. 


\subsection{RELIABLE MESSAGING}

\subsubsection{PROBLEM}

For real world problems, the reliable transfer of data is very important which otherwise may result in unpleasant consequences. There is trade-off between using of reliable messaging and performance as there is good deal of performance cost associated as describe in following sections. Data may still be lost after being received due to power failure, memory corruption etc. Further some processes may take longer to complete which makes them more prone to such undesirable situations.

\subsubsection{SOLUTION}

Reliable Messaging (Earl, 2009) addresses this problem by introducing an intermediate database to persist the arrived message. The pattern implementation is provided natively by the host platform and is not under direct control of developer.

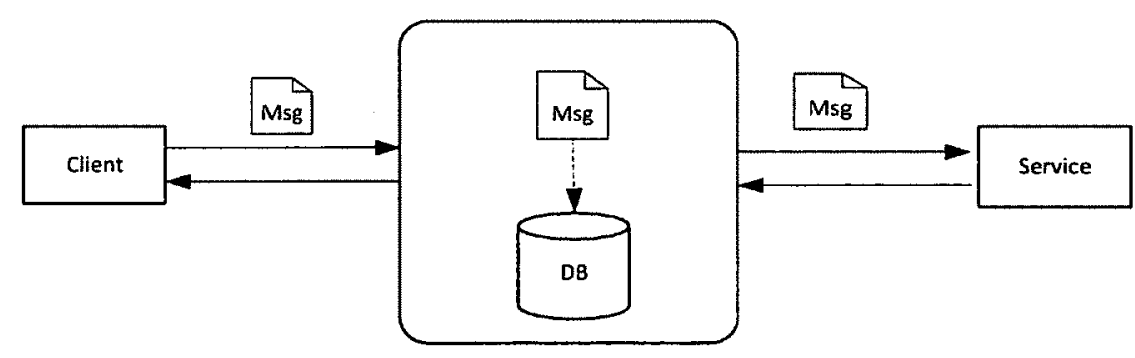

Figure 2 Reliable Messaging Pattern

\subsubsection{APPLICATION}

The following simplified diagram helps to describe the application of Reliable Messaging pattern in Biztalk server. 


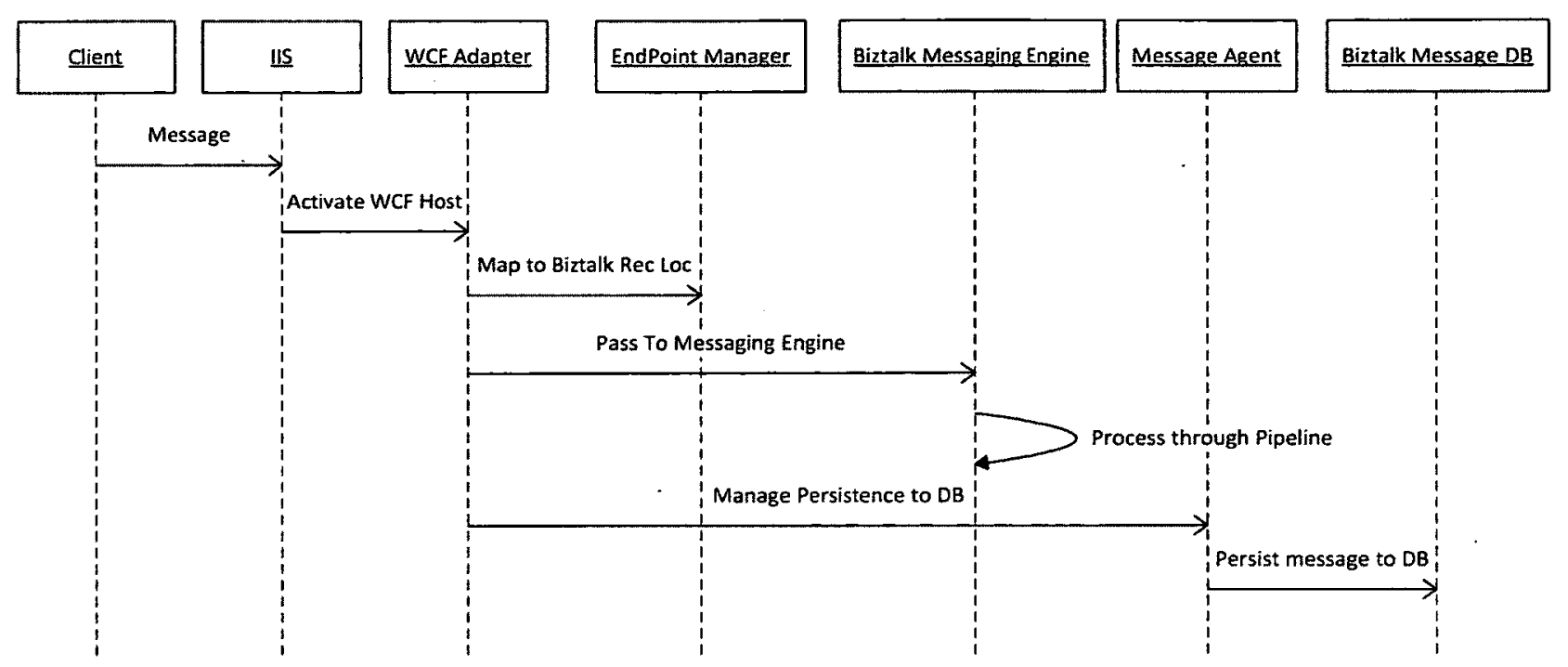

Figure 3 Native reliable message implementation provided by Biztalk

- The client sends message to endpoints hosted by a web server called Internet Information Services (IIS) using public Uniform Resource Locator URL.

- IIS create instance of the end point adapter (WCF endpoint).

- Adapter calls the Biztalk Endpoint Manager Service to retrieve the settings of the receive location that matches the WCF endpoint hosted by IIS. It provides a map from WCF endpoint to Biztalk Receive locations and applies any configurations.

- The message is passed to Biztalk Messaging Engine which applies pipeline such as XMLReceive to raw message.

- Finally the message Agent, which is a service to interface with MessageBox DB, writes the message to DB. 
The adapter could application specific such as SQL Server, SAP, ORACLE or transport protocol adapter such as Microsoft Messaging Queue (MSMQ), Hyper Text Transfer Protocol (HTTP), SOAP or FILE.

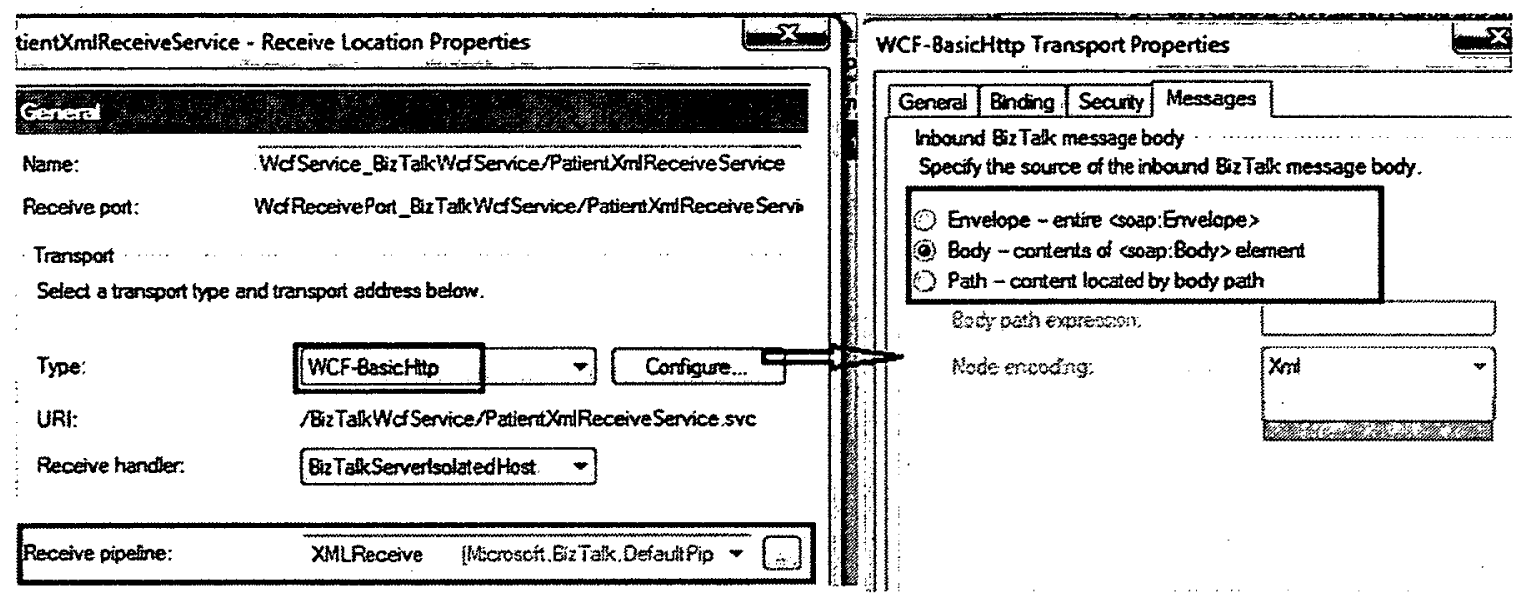

Figure 4 Biztalk WCF Adapter configuration of SOA

\subsection{PIPES AND FILTERS FOR MESSAGING}

\subsubsection{PROBLEM}

The message may need to be decrypted, decompressed or any other processing may be required on message such as XML parsing or breaking a composite message into several pieces before delivering it to the recipient.

\subsubsection{SOLUTION}

Use a messaging processing pipeline (Hohpe and Woolf, 2003) where each stage of the pipeline performs a specific task and delivers to the next stage if further processing is required. 


\subsubsection{APPLICATION}

Biztalk server provides two default pipelines, namely PassThru which accepts and deliver message in its raw format without any changes, if needed, and XML Receive for XML parsing. The incoming message is handed over to an adapter followed by processing through a message pipeline. Note that this is a very specialized application of the pipes and filters pattern, which in principle can be used for other purposes than messaging.
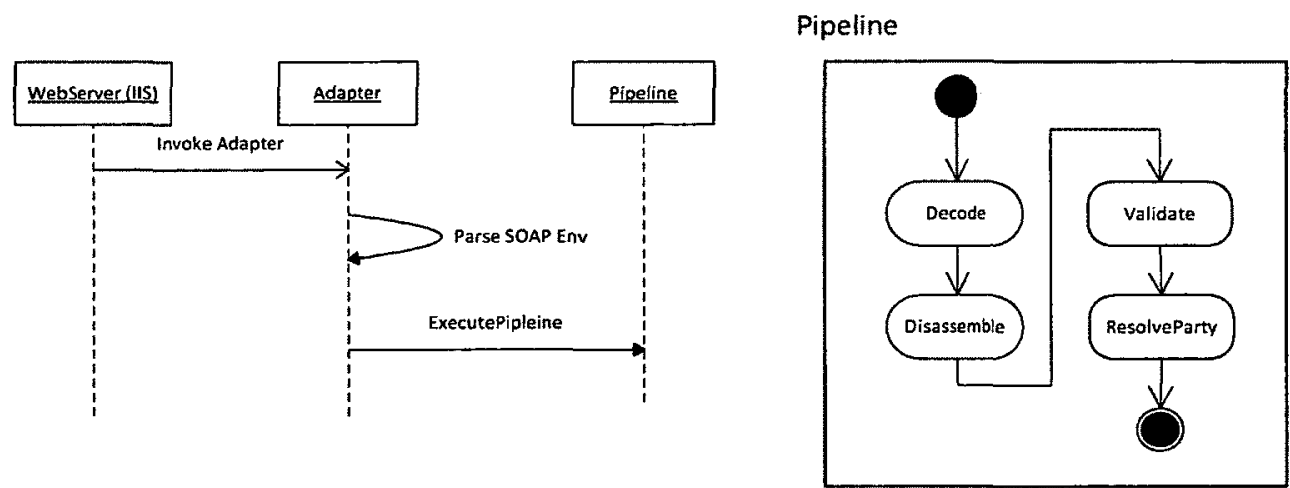

\section{Figure 5 a) Pipes and Filter Invocation b) Biztalk Default Pipeline Stages}

Pipeline is responsible for:

- Decoding the message if it is encrypted and/or compressed.

- Next stage is Disassemble where message is converted to XML for processing inside Bizalk domain and at same time identifying its type which is then promoted to message context or in other words attached to message.

- Following is Validate stage which can be used to validate contents of XML.

- Final stage is ResolveParty, which can route the message based on the address of sender without looking into contents. 


\subsection{PUBLISH/SUBSCRIBE}

\subsubsection{PROBLEM}

There could be many messages arriving at the same time and various parties expecting to receive them. For example, Process-A expect to receive MsgType-1 and Process-B to receive MsgType-2 where as there is another Process-C that expects to receive both MsgType-1 and MsgType-2.

\subsubsection{SOLUTION}

Instead of point to point communication between service and sender, publish-subscribe (Hohpe and Woolf, 2003) introduces and intermediary between communicating parties. The sender can submit a message to a central repository and the service will receive the message if it has been designated to receive that type of message. This way multiple parties can subscribe to a particular message type.

\subsubsection{APLLICATION}

Biztalk server uses publish-subscribe pattern to deliver messages arriving into MessageBox Database (DB) to orchestrations who subscribed to them. At design time, the developer creates a property schema (an xsd file) used by Biztalk messaging engine on runtime to match elements in the incoming XML to those contained in the property schema. When it finds a match, those properties are promoted into message context, i.e., are explicitly attached to the message being forwarded to Message Box DB.

Figure 6 shows the creation Orchestration and Port (particular schema) subscription, which writes an entry into Biztalk Subscription database. 


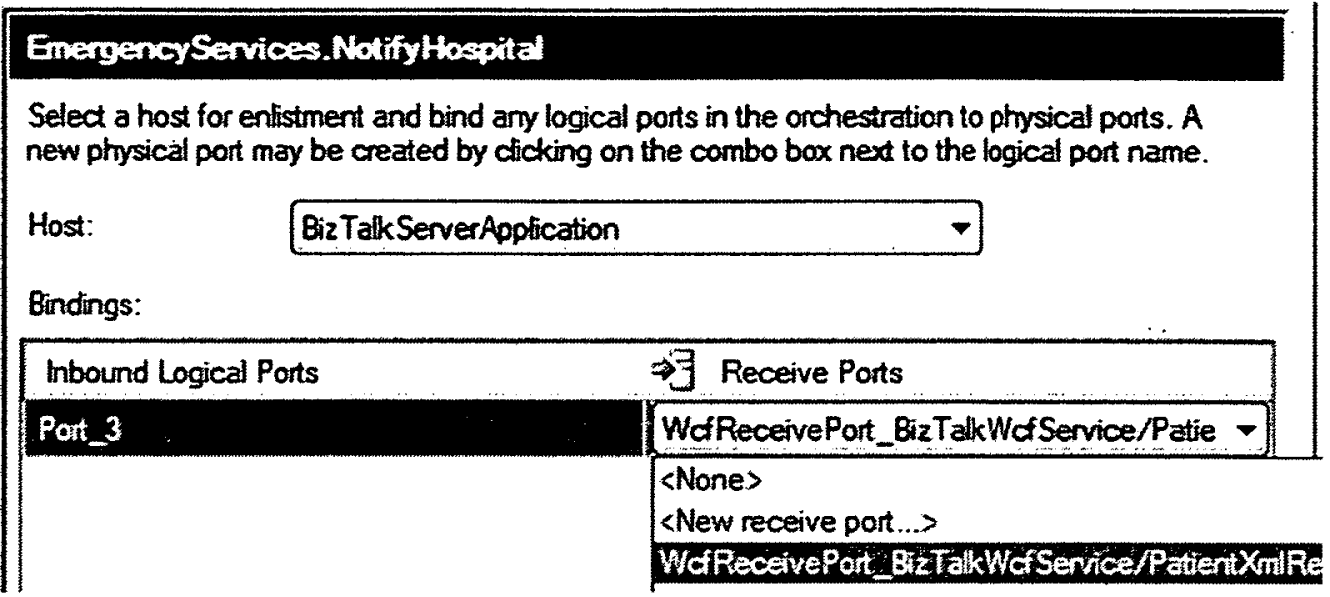

Figure 6 Creation of a Subscription in Biztalk

As shown in the simplified sequence diagram in Fig. 5.a, the Message Agent writes Message to MessageBox. Before it does that, it asks MessageBox to find the subscription of the message in question. MessageBox then queries the subscription database and links the subscription results to the message. The Message Agent along with subscription details inserts the message into MessageBox DB. MessageBox is implemented as First In First Out (FIFO) and Biztalk Engine has multiple de-queuing threads that continuously poll the DB; one thread will eventually pick up the message from the queue and deliver it to the subscribed orchestration instance or create one if doesn't already exist.

\subsubsection{DEHYDRATION AND REHYDRATION}

When Biztalk determines whether an orchestration instance has been idle for a long period, and if it's active, it Dehydrates the instance. This happens by saving the state of orchestrations instance, and releasing the memory resources. This makes possible for a large number of long-running orchestrations to run concurrently on the same computer.

Rehydration is the reverse of Dehydration, by which the engine loads a saved instance context into memory and restores its state. The orchestration then continues to run from the point where it was Dehydrated. 


\subsection{CORRELATION IDENTIFIER}

\subsubsection{PROBLEM}

Data or messages may originate from various sources but need to be processed together by a particular business process. A mechanism is required to identify what subset of arriving messages from various sources together needs to be correlated together.

\subsubsection{SOLUTION}

A correlation set (Hohpe and Woolf, 2003) is data unique to a message or set of messages that can be used to tie together the messages that needs to be processed together by a particular process instance.

In our case study, the information required by the healthcare provider may require information about patient from various sources. This information needs to be integrated as unified message inside the workflow system before being sent to hospital. For example, the emergency site provides patient basic info including the health card number. The health card number forms the basis for correlation set as information arriving from other sources will also provide the same health card number.

Figure 7 shows the sequence of steps performed in correlation identification process with aid on an activity diagram. It also accounts for any orchestration in dehydrated state when subsequent message required for correlation arrive. 
Figure 7 Correlation Identifier Process

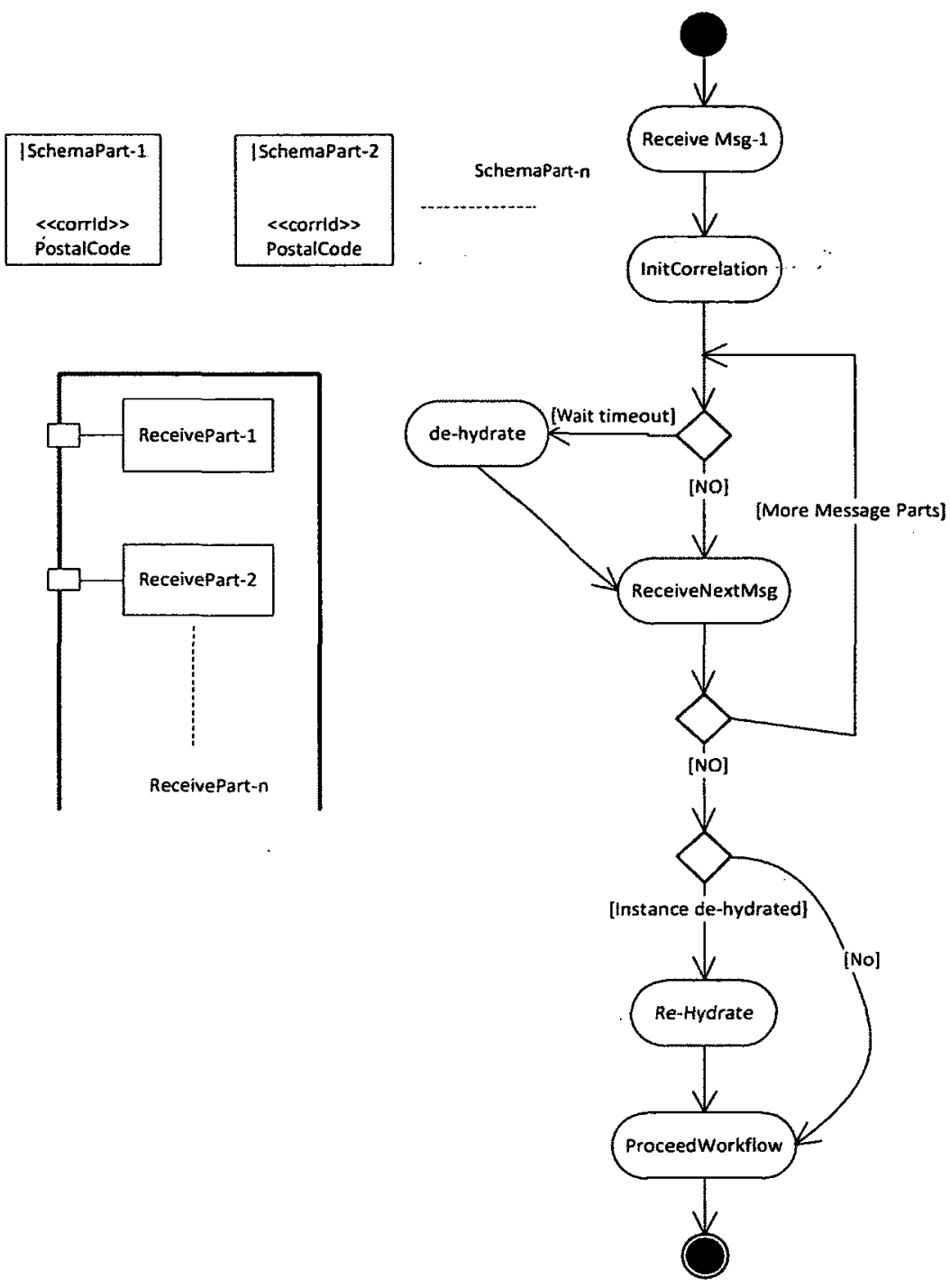

This is particularly useful when information arrives into system simultaneously regarding multiple patients which be erroneously integrated with another patient's information before being sent to emergency care provider.

Figure 8 (a) shows the attributes which are used to perform the correlation from two message instances where as Figure 8 (b) shows the actual correlation properties used at design time in Biztalk Server. 


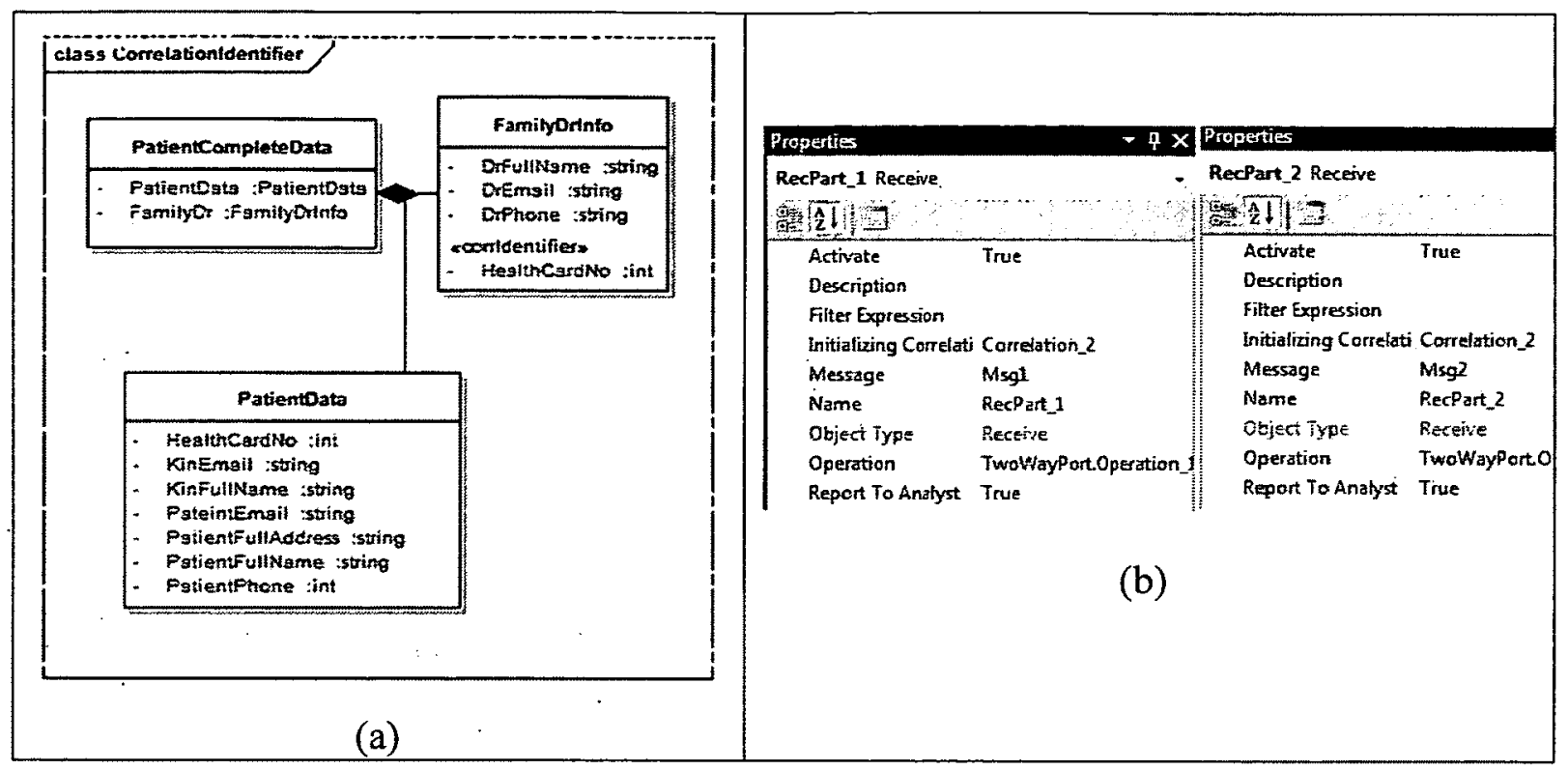

Figure 8 (a) Correlation attributes. (b) Correlation properties in Biztalk

We need to mention one of the biggest challenges during our experimental part of the research: the lack of good measurement tools. The testing tools soapUi and loadUI measure end to end response time (between the sending of a message and receiving a reply) without any flexibility to measure time required to perform the correlation only. So, we were unable to measure the separate contribution to the overhead of these separate patterns, only the overall cumulated effect.

\subsection{PERFORMANCE IMPACT OF PLATFORM-PROVIDED PATTERNS}

All of the patterns and their implementation described above are basic building block of a messaging system implemented by Biztalk. To measure the performance of each in isolation is very difficult, we therefore came up with a strategy to implement an empty workflow exposed as a SOA web service that employs pipeline, message persistence and subscription feature as described above in the publish subscribe patterns. The pipeline, 
message persistence and subscription feature implementation are hidden from the developer writing the application that invokes a workflow service.

Figure 9 Approach for estimating

the overhead introduced by platform-provided patterns

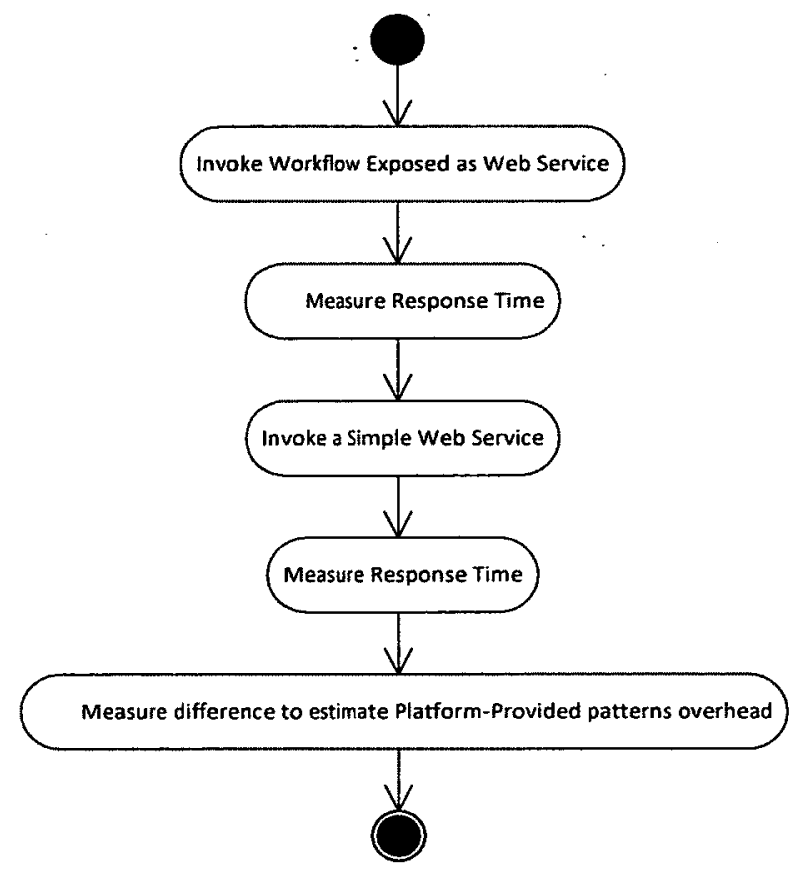

We then created a simple SOAP web service, which does not involve any pipeline and publish subscribe mechanism and called its operation using our testing tools. Both services are exposed to outside world as a SOAP service via a WSDL interface but the former provides the implementation of pipeline, message persistence and subscription behind the scenes. We then invoked both services individually 180 times in a 60 minutes time span and measured the results. This process is explained in Figure 9.

\subsubsection{INVOCATION OF A WORKFLOW SERVICE}

The following figure shows how the invocation of an empty workflow service is represented by the BizTalk developer tool. The workflow service does not contain any business logic, other than receiving a request and sending a reply, but still it incurs the overhead implemented behind the scenes by the platform due to the application of 
Publish-Subscribe, Reliable Messaging, Pipes and Filters. It also has the overhead of SOAP serialization and de-serialization as found in any SOAP based web service.

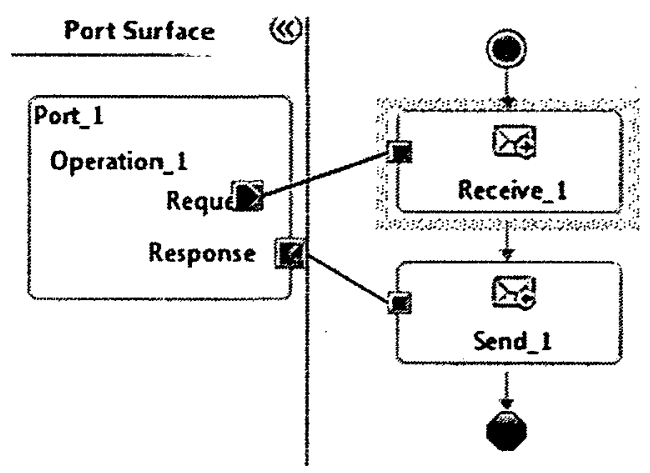

Figure 10 Empty workflow service implemented in Biztalk

\subsubsection{INVOCATION OF A SIMPLE SERVICE}

The implementation of a simple conventional SOAP-based service is very much like a method implementation of a class in an object oriented language. Each class, later exposed as a service, can have various methods which are then exposed as web service operations in the WSDL description generated for the web service clients. This simple web service has been implemented in order to compare its invocation response time with that of the workflow service. The following figure shows the implementation of a simple web service which looks identical to any other function or method definition. 


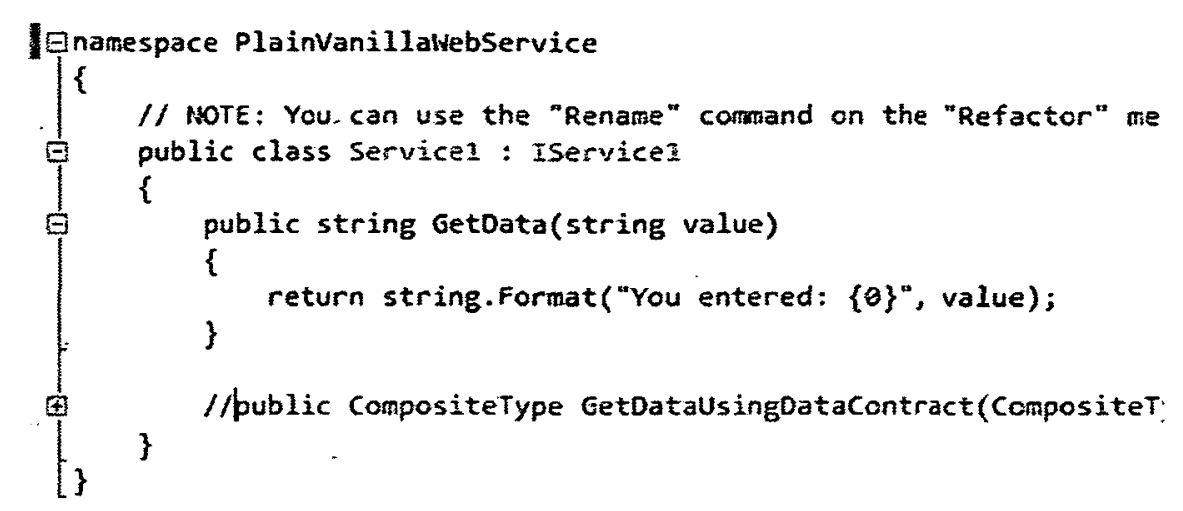

Figure 11 A simple SOAP based web service implementation

\subsubsection{PERFORMANCE OVERHEADS OF WORKFLOW SERVICE AND SIMPLE SERVICE INVOCATION}

Figure 12 shows the response time for an empty workflow service as described in the section 3.6.1. The measurements were repeated for 180 invocations over a period of 60 minutes. Test was performed with $1 \mathrm{~KB}$ message size. The response time results are shown in graph below; the average response time is $644.42 \mathrm{~ms}$. The fluctuation in measurements is due to the fact that, despite terminating all un-wanted processes, some system level processes that cannot be terminated at will interfere with the measurements.

\section{Data Size $=1 \mathrm{~KB}:$}

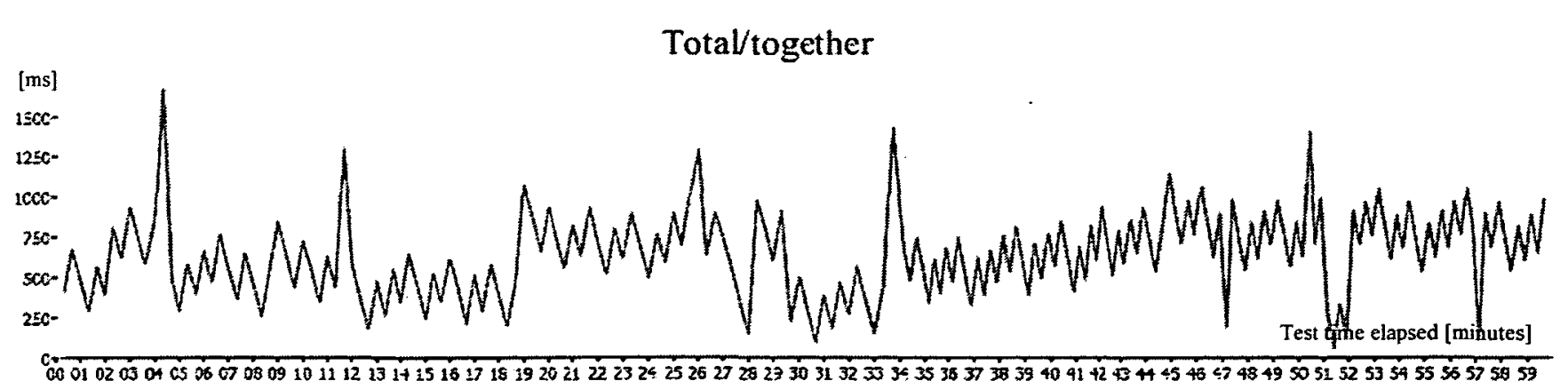

Figure 12 Response Time Measurement of an empty workflow service invocation 
As we can see, the variations in response time values are quite large, due to the complex processing by the patterns implemented by the tool behind the scenes, which offer many alternative execution paths and reasons for variations.

Figure 13 shows the response time for 180 repeated invocations of an empty simple web service over a period of 60 minutes. The message size was of $1 \mathrm{~KB}$. The graph is smoother than for the invocation of a workflow service from Figure 12, because the processing is simpler, with fewer alternative execution paths and reasons for variation.

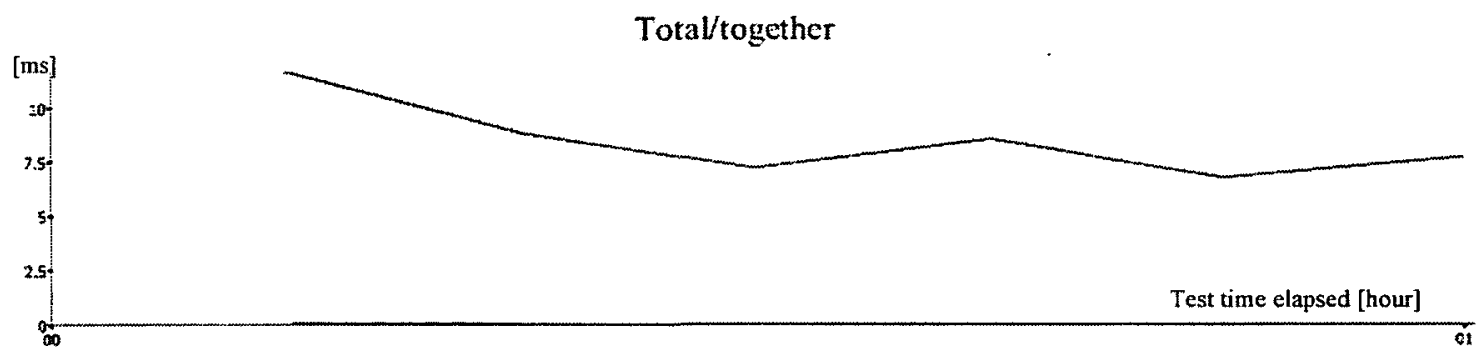

Figure 13 Response Time Measurements of a simple SOAP service invocation

Comparing the invocation response times of a workflow service with that of a simple service, we can see that the first is considerably longer than the second (see table 2).

Table 2 Comparing Average Response time for workflow and simple service invocation

\begin{tabular}{|l|c|}
\hline Operation (end to end) & Average Response Time (ms) \\
\hline Workflow Service invocation & 644.42 \\
\hline Simple Service invocation & 8.54 \\
\hline
\end{tabular}

The reasons for the large difference of approx. $636 \mathrm{~ms}$ between the two kinds of 
invocations can be found by looking at the difference of what is executed behind the scenes in each case. The response time of a workflow service invocation is an accumulation of the following overheads:

- Form a SOAP envelope.

- Send across to the receiving end point (in this case no network delay is involved as test is done on a single computer).

- Parse the SOAP envelope to remove the header;

- Process through pipeline which in this case is a deserialization of XML. Further pipeline steps such as decryption and decompression if applied by sender, will further increase response time.

- Persist the message to MessageBox DB, which is default behavior when Biztalk receives any message.

- Decipher subscription of message and creation of orchestration instance to which message is then delivered. This ends the Receive block.

- The send block creates a SOAP response and sends back to client.

- Parsing of SOAP envelope by the client (this operation on the client side is also included in the response time measurement).

On the other hand, a simple service invocation includes the overhead of the SOAP protocol. The response of a simple conventional service invocation includes the following overheads:

- Form a SOAP envelope.

- Send across to the receiving end point (in this case no network delay involved as the test is done on a single computer). 
- Parse the SOAP envelope to remove the header.

- Create a SOAP response and send back to client.

- Parsing of SOAP envelope by the client.

The operations that are common to the two cases are: the creation and sending a SOAP envelope, parsing envelope on receiving end and send a reply back to the caller. There is no other business logic implemented in either the simple web service or in the workflow; both are just relaying a $1 \mathrm{~KB}$ message across the two ends.

The significant difference of almost two orders of magnitude between the two kinds of invocations is due to the overhead associated with the hidden patterns implemented by the service platform: pipeline and filters, message persistence and subscription resolution.

\subsection{PERFORMANCE IMPACT OF MESSAGE SIZE}

Another factor impacting performance is the size of message being transmitted. We performed experiment to invoke a workflow by sending a message with variable data sizes and receiving a reply back with the same size. We used our workflow exposed as web service to measure variable data size impact. These results are provided below. For consistency, we mention once again the results from previous test for $1 \mathrm{~KB}$.

\subsubsection{Response Time Measurements for $1 \mathrm{~KB}$}

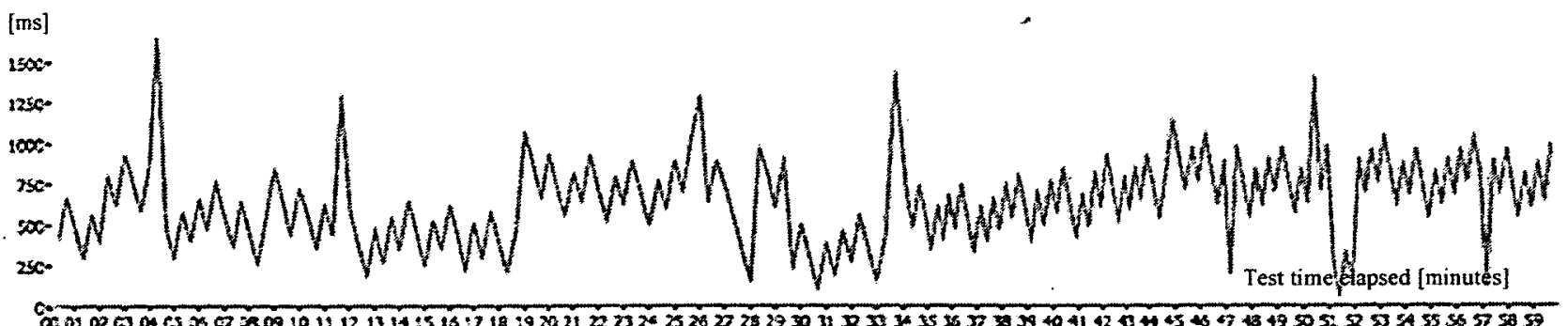

Figure 14 Response Time Measurements for $1 \mathrm{~KB}$ 
Table 3 Average Response Time (1 KB)

\begin{tabular}{|c|c|}
\hline Data Size (KB) & Average Response Time (ms) \\
\hline 1 & 644.41 \\
\hline
\end{tabular}

\subsubsection{Response Time Measurements for $10 \mathrm{~KB}$}

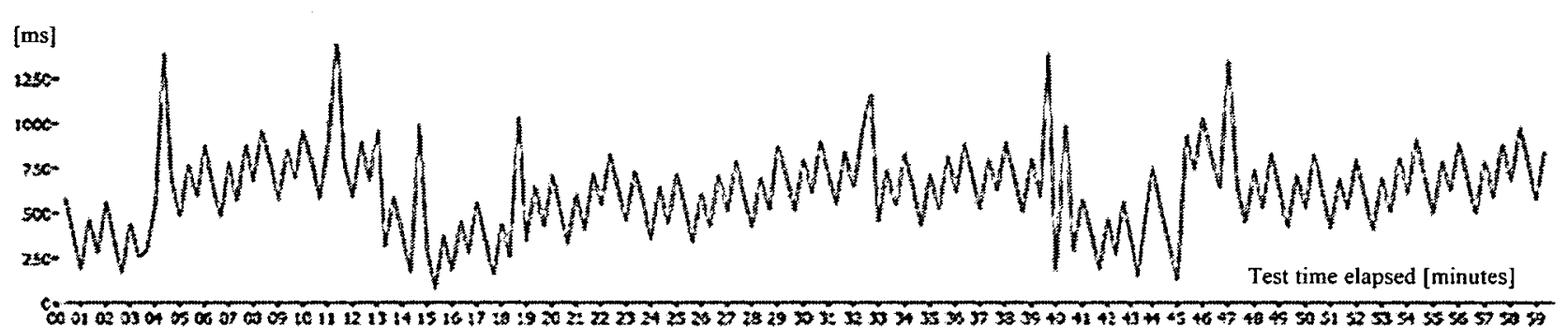

Figure 15 Response Time Measurements for $10 \mathrm{~KB}$

Table 4 Average Response Time (10 KB)

\begin{tabular}{|c|c|}
\hline Data Size (KB) & Average Response Time (ms) \\
\hline 10 & 682.27 \\
\hline
\end{tabular}

\subsubsection{Response Time Measurements for $100 \mathrm{~KB}$}

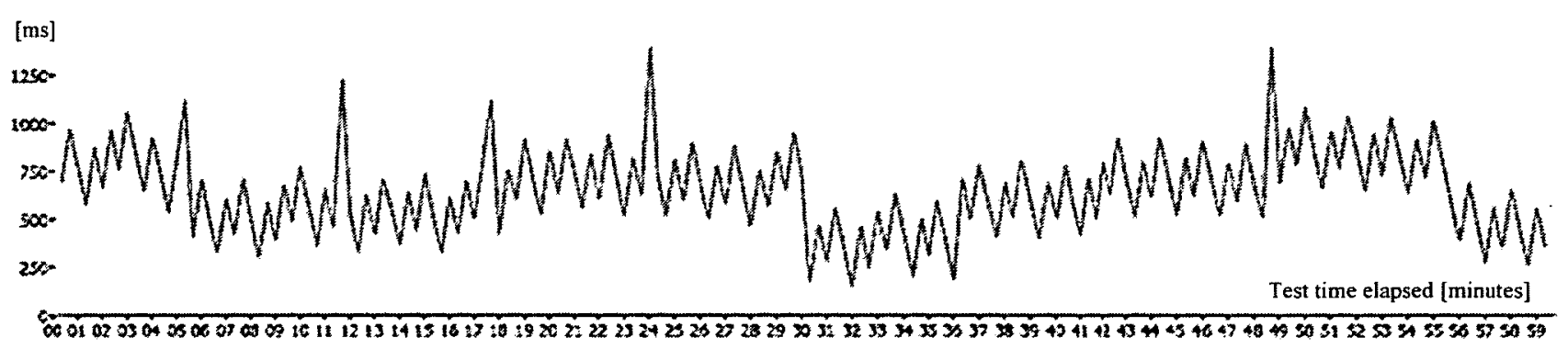

Figure 16 Response Time Measurements for $100 \mathrm{~KB}$

Table 5 Average Response Time $(100 \mathrm{~KB})$

\begin{tabular}{|c|c|}
\hline Data Size (KB) & Average Response Time (ms) \\
\hline 100 & 698.03 \\
\hline
\end{tabular}




\subsubsection{Response Time Measurements for $200 \mathrm{~KB}$}

\section{Response Times}

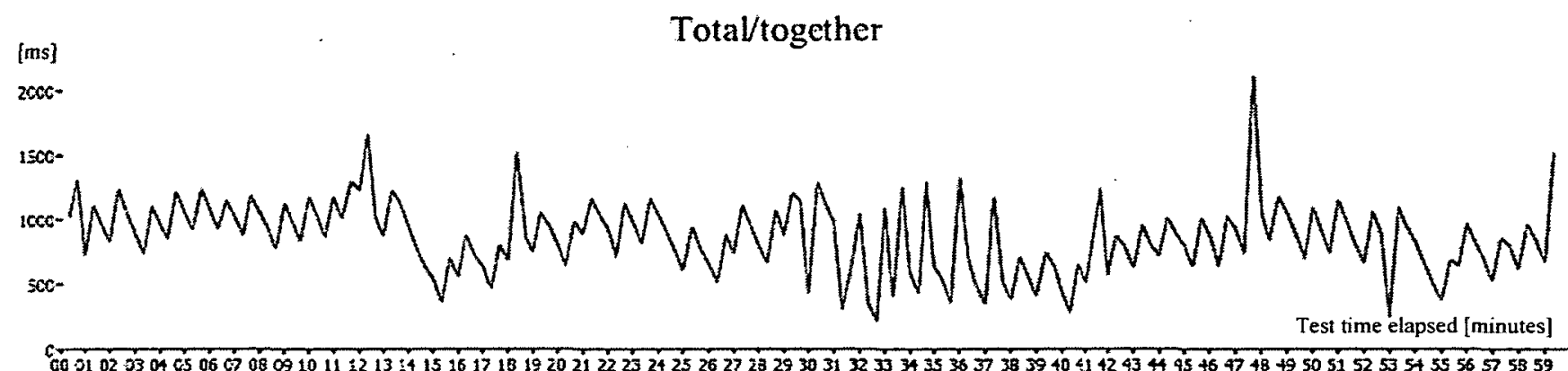

Figure 17 Response Time Measurements for $200 \mathrm{~KB}$

Table 6 Average Response Time (200 KB)

\begin{tabular}{|c|c|}
\hline Data Size (KB) & Average Response Time (ms) \\
\hline 200 & 727.10 \\
\hline
\end{tabular}

\subsubsection{Response Time Measurements for $400 \mathrm{~KB}$}

\section{Response Times}

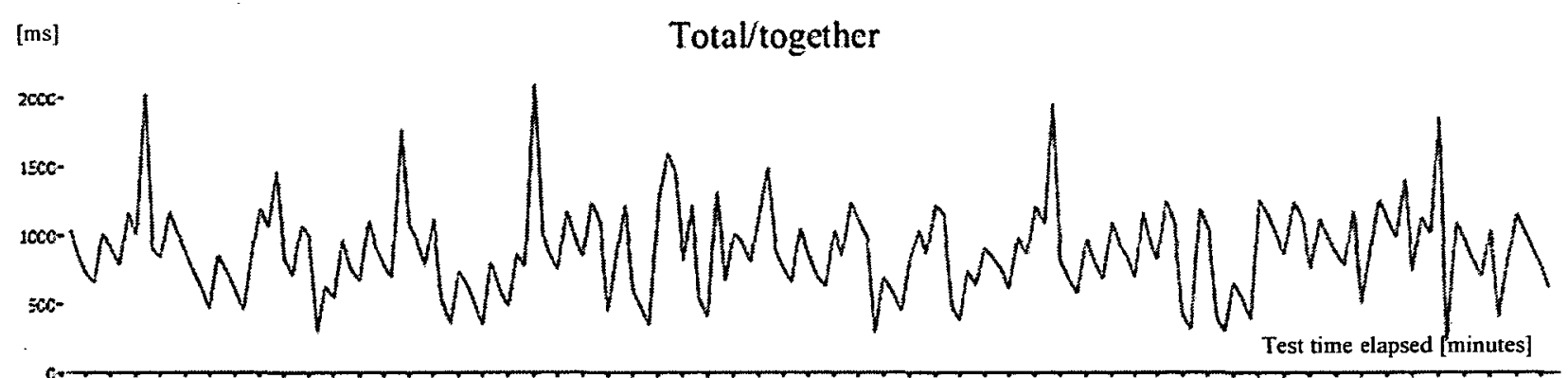

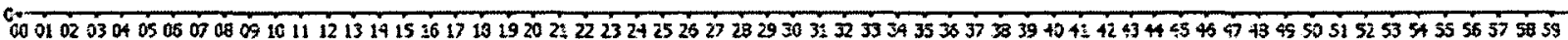

Figure 18 Response Time Measurements for $400 \mathrm{~KB}$

Table 7 Average Response Time (400 KB)

\begin{tabular}{|c|c|}
\hline Data Size (KB) & Average Response Time (ms) \\
\hline 400 & 793.12 \\
\hline
\end{tabular}




\subsubsection{Response Time Measurements for $600 \mathrm{~KB}$}

\section{Response Times}

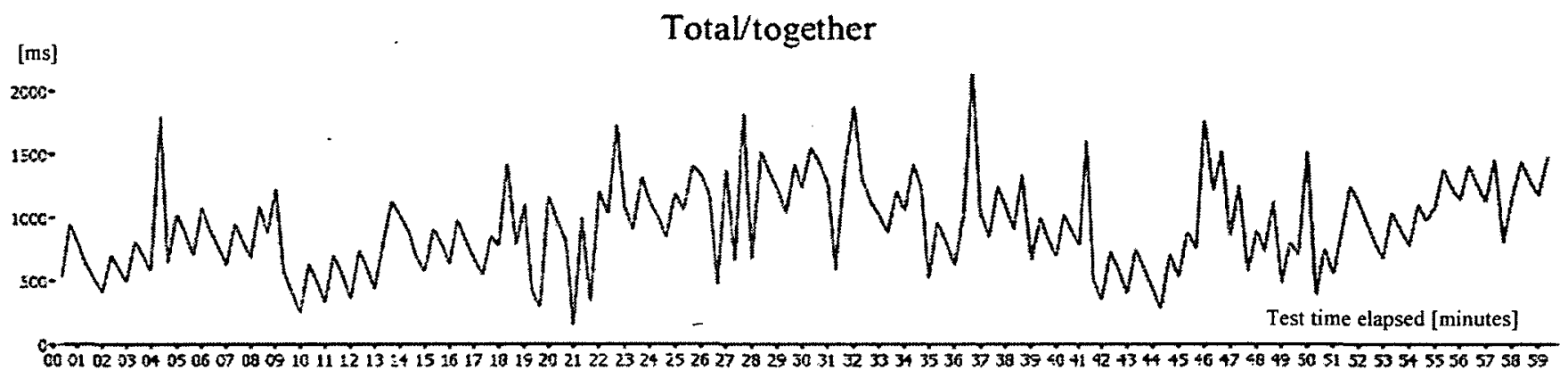

Figure 19 Response Time Measurements for $600 \mathrm{~KB}$

Table 8 Average Response Time (600 KB)

\begin{tabular}{|c|c|}
\hline Data Size (KB) & Average Response Time (ms) \\
\hline 600 & 877.39 \\
\hline
\end{tabular}

\subsubsection{Response Time Measurements for $800 \mathrm{~KB}$}

Response Times

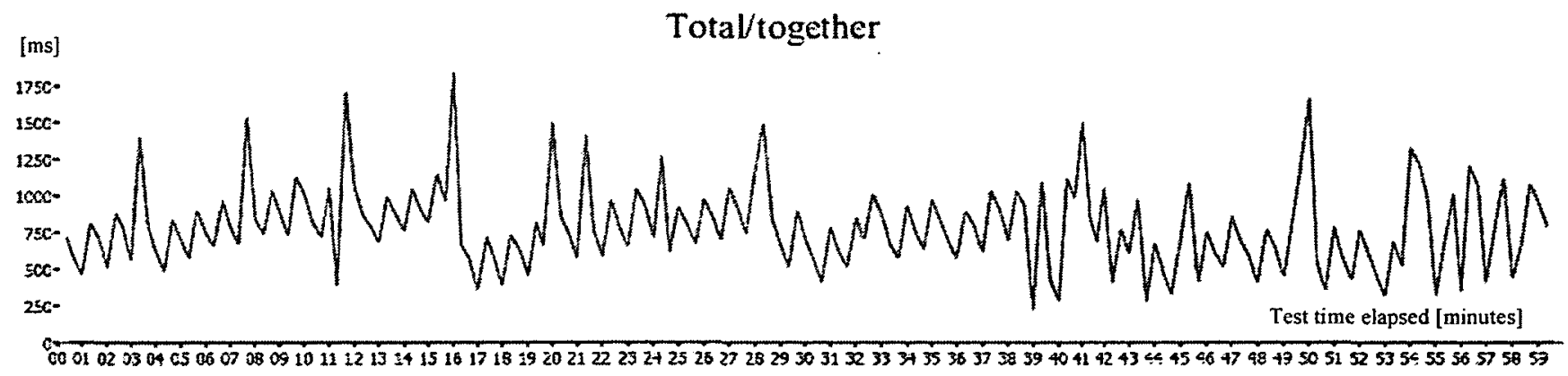

Figure 20 Response Time Measurements for $800 \mathrm{~KB}$

Table 9 Average Response Time ( $800 \mathrm{~KB}$ )

\begin{tabular}{|c|c|}
\hline Data Size (KB) & Average Response Time (ms) \\
\hline 800 & 904.22 \\
\hline
\end{tabular}




\subsubsection{Response Time Measurements for $1000 \mathrm{~KB}$}

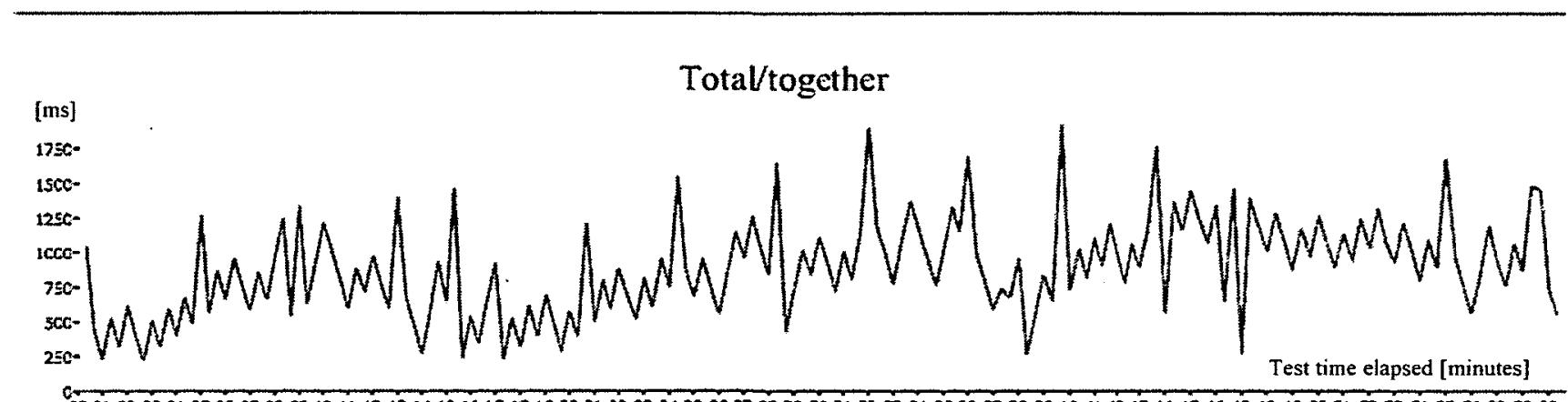

Figure 21 Response Time Measurements for $1000 \mathrm{~KB}$

Table 10 Average Response Time (1000 KB)

\begin{tabular}{|c|c|}
\hline Data Size $(\mathrm{KB})$ & Average Response Time (ms) \\
\hline 1000 & 939.12 \\
\hline
\end{tabular}

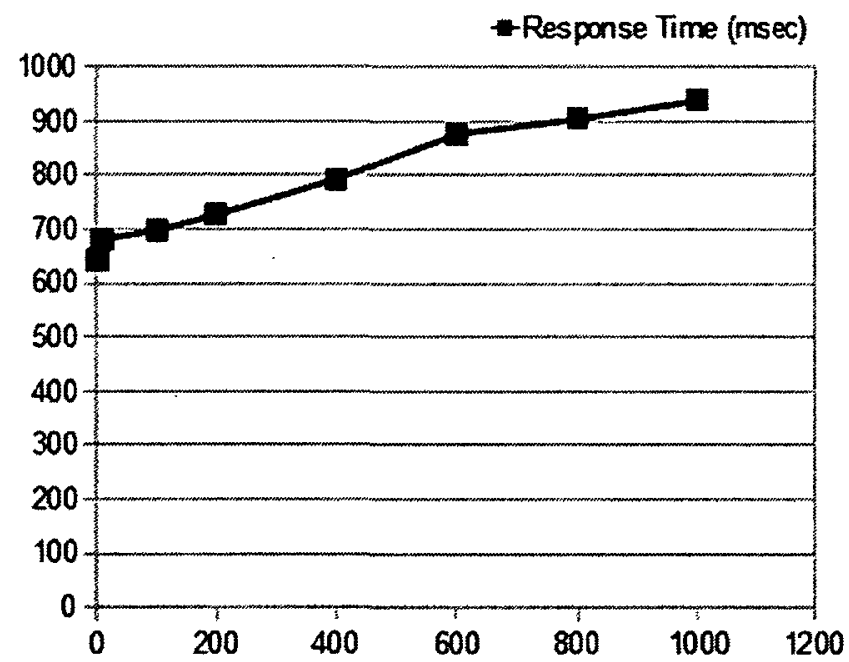

Figure 22 Response Time Versus message size [KB]

The message size has an impact on the response time for the invocation of an empty workflow service, as it can be seen from plotted graph in Figure 22. The response time increases from $650 \mathrm{~ms}$ for $1 \mathrm{~KB}$ to about $930 \mathrm{~ms}$ for $1000 \mathrm{~KB}$. The actual processing of 
the message content for a $1 \mathrm{~K}$ message has very small effect on response time, as most of the time is spent in various stages of the platform-provided patterns. We draw this conclusion from the fact that the measured response time for a $2 \mathrm{~K}$ message for the same workflow was almost the same as for $1 \mathrm{~K}$. Hence we can assume that $650 \mathrm{~ms}$ delay is the performance penalty. paid by the invocation of a workflow service on the computer configuration we used for measurements. This overhead will be used in Chapter 5 for the estimation of the end-to-end response time in the design phase of service-based applications. 


\section{PERFORMANCE MEASUREMENTS OF APPLICATION-LEVEL PATTERNS}

\subsection{INTRODUCTION}

As mentioned earlier, some SOA patterns are implemented by the platform and are hidden from the developers, while others are applied explicitly by developers in an environment such as the Biztalk server. In this section we will investigate the performance effects of the latter category of patterns. We will describe these patterns using UML notation and will apply them to our case study.

The starting point for our case study is a manual healthcare process for emergency situations involving senior residents at homes for the elderly. In emergency cases, the staff at such facilities needs to call the paramedics to transfer a patient to a hospital. The details of the performed procedures such as CPR and other information need to be relayed to the hospital. The emergency staff and paramedics fill out information on paper that includes patient personal information, CPR and medication related information such as allergies etc. This manual process is time consuming but it is critical to providing required medication to patient inside or in transit to hospital.

We proposed an automated solution for handling such emergency cases as a business process, which itself can be invoked as a SOAP service by the residence staff. This process receives the necessary information about the patient through XML messages according to the service interface and in turn invokes another service provided by the hospital for relaying information about emergency transfers.

Our objective is to measure the performance of a workflow (such as its end-to-end response time) after implementing these patterns to solve a business problem. 
Performance of web service based systems is affected by some inevitable building blocks provided by the tool, which were described in Chapter 2, over which the designer has very limited control.

Our approach to measuring system performance after using application level pattern in order to design and implement a SOA application has two granularity levels:

1. Measure end to end response time, i.e. time required from the invocation of the workflow by a client to its completion, when it sends a response back to the client.

2. Measure the execution time for each step of the workflow to get better insight, were the time is spent; for this we have used the orchestration profiling tool that provide break down of time required to complete each step.

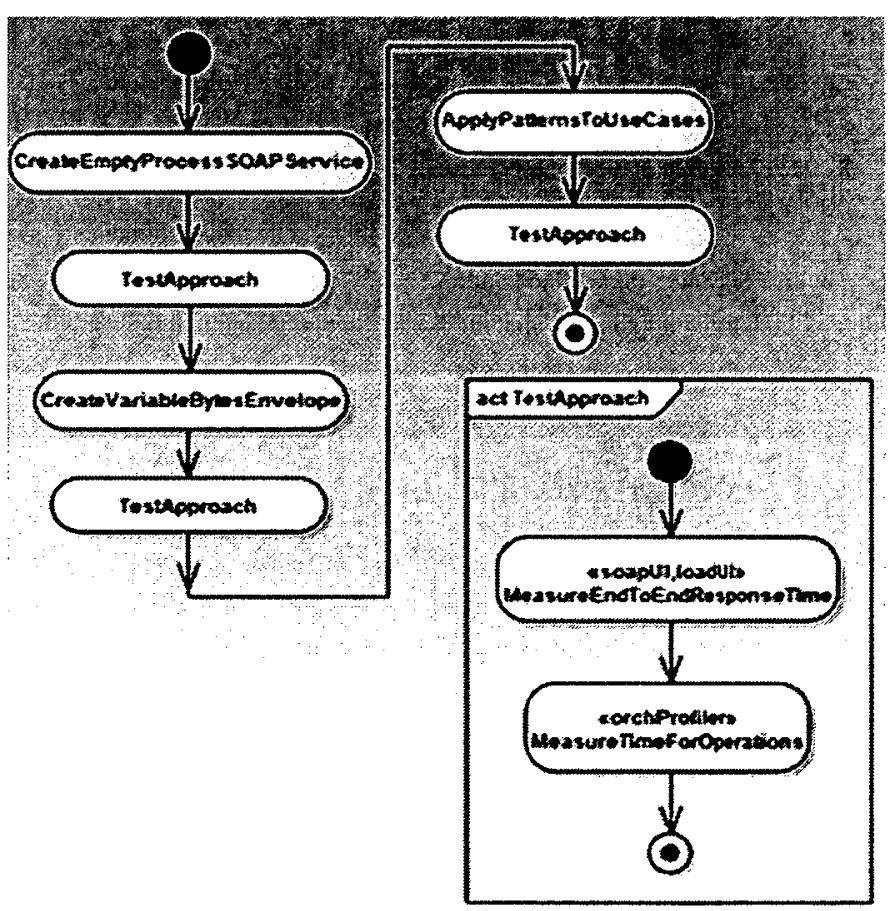

Figure 23 Approach followed in Testing 
We built our system by incrementally adding these building blocks to the system as indicated in Figure 23 and measuring the response time according to approaches mentioned above. Due to the fact that the response time is a stochastic variable, every measurement was repeated between 180 and 240 times, and the total duration of an experiment was of about 1 hour.

With a basic idea of some default overhead in implementing our design as evaluated in previous chapter, we started incorporating the use case of our case study by applying the appropriate SOA patterns.

Table 11 lists the application-level pattern investigated in this chapter. We have focused mainly on messaging patterns, because they address the same kind of problems as the platform-provided patterns discussed in the previous chapter.

Table 11 Development Patterns Application to Case Study

\begin{tabular}{|l|l|}
\hline \multicolumn{1}{|c|}{ SOA Pattern Utilized } & \multicolumn{1}{c|}{ Use Case Implemented } \\
\hline Message Translator & $\begin{array}{l}\text { Patient data arriving could be in one format } \\
\text { whereas the final recipient (hospital) expects } \\
\text { logically equivalent data but in other format }\end{array}$ \\
\hline Aggregator - Sequential & Integrate patient related data, arriving in a \\
defined sequence from various sources, inside \\
workflow and dispatch integrated data to \\
emergency care provider
\end{tabular}




\begin{tabular}{|l|l|}
\hline & $\begin{array}{l}\text { and dispatch integrated data to emergency care } \\
\text { provider }\end{array}$ \\
\hline Content Based Router & $\begin{array}{l}\text { Allow the client to choose emergency care } \\
\text { provider and supply their Web Service URL }\end{array}$ \\
\hline Scatter and Gather & $\begin{array}{l}\text { Allow workflow to solicit current patient load } \\
\text { from care providers in region, forward non- } \\
\text { critical cases to less busier locations }\end{array}$ \\
\hline
\end{tabular}

\subsection{MESSAGE TRANSLATOR}

\subsubsection{PROBLEM}

One of the major challenges in the SOA world is a different way of storing and transmitting information. XML is a common way of transmitting information in Web Services based system but parties involved in sending and receiving of messages may use different design of XML schemas that logically contain the same piece of information.

\subsubsection{SOLUTION}

In order for these systems to work and integrate together the workflow system acting as a mediator, it should able to perform the message translation or data transformation (Earl, 2009 ) in both directions i.e. transform message into format required by workflow and then send a response in format required by the client. The pattern is shown in Fig 24.

\subsubsection{APPLICATION}

In our case study, the client sending the patient's and other related information is using a proprietary design of XML schema and the hospital Web Service exposed to receive this 
logically equivalent information may be using a different XSD schema. This message translation therefore becomes the responsibility of the orchestrating system and requires system resources. Figure 25 shows pattern applied in Biztalk and Figure 26 shows two message structures which are different but logically express the same information.

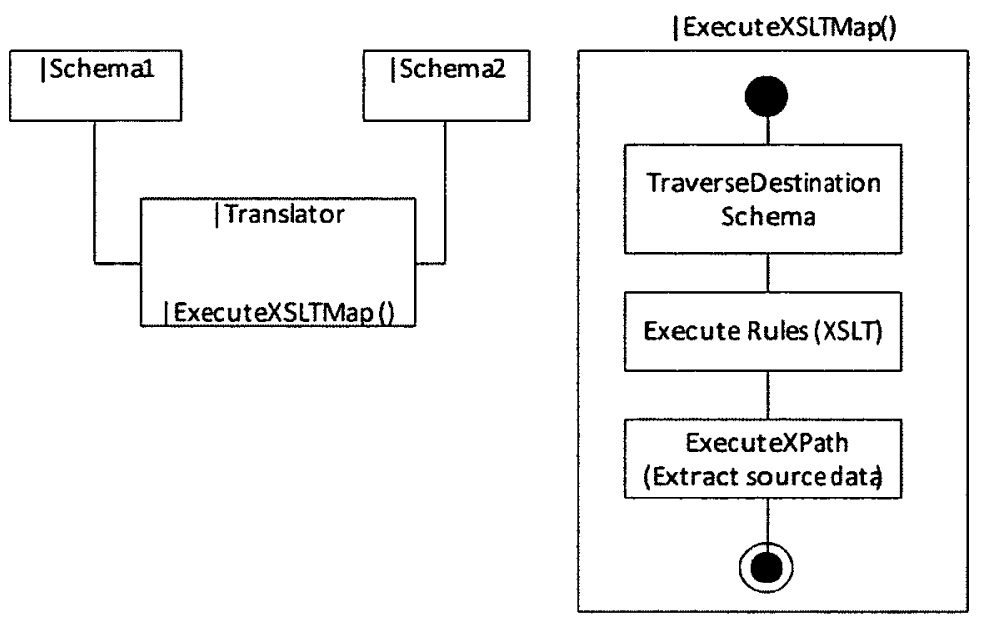

Figure 24 Message Translator Pattern

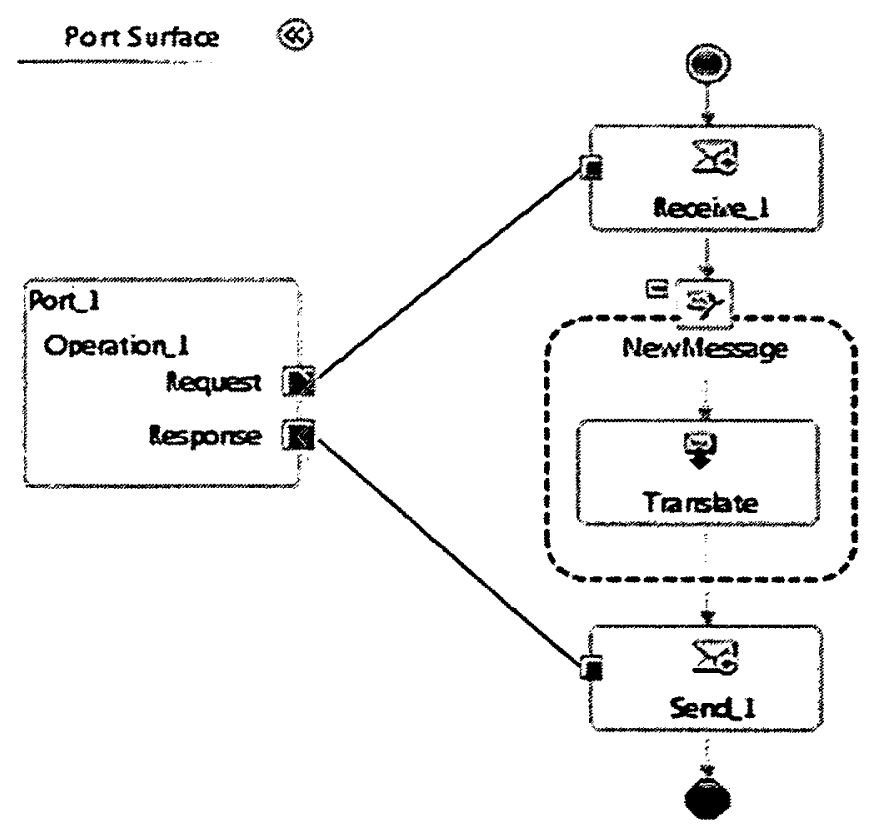

Figure 25 Message Translator implemented in Biztalk 


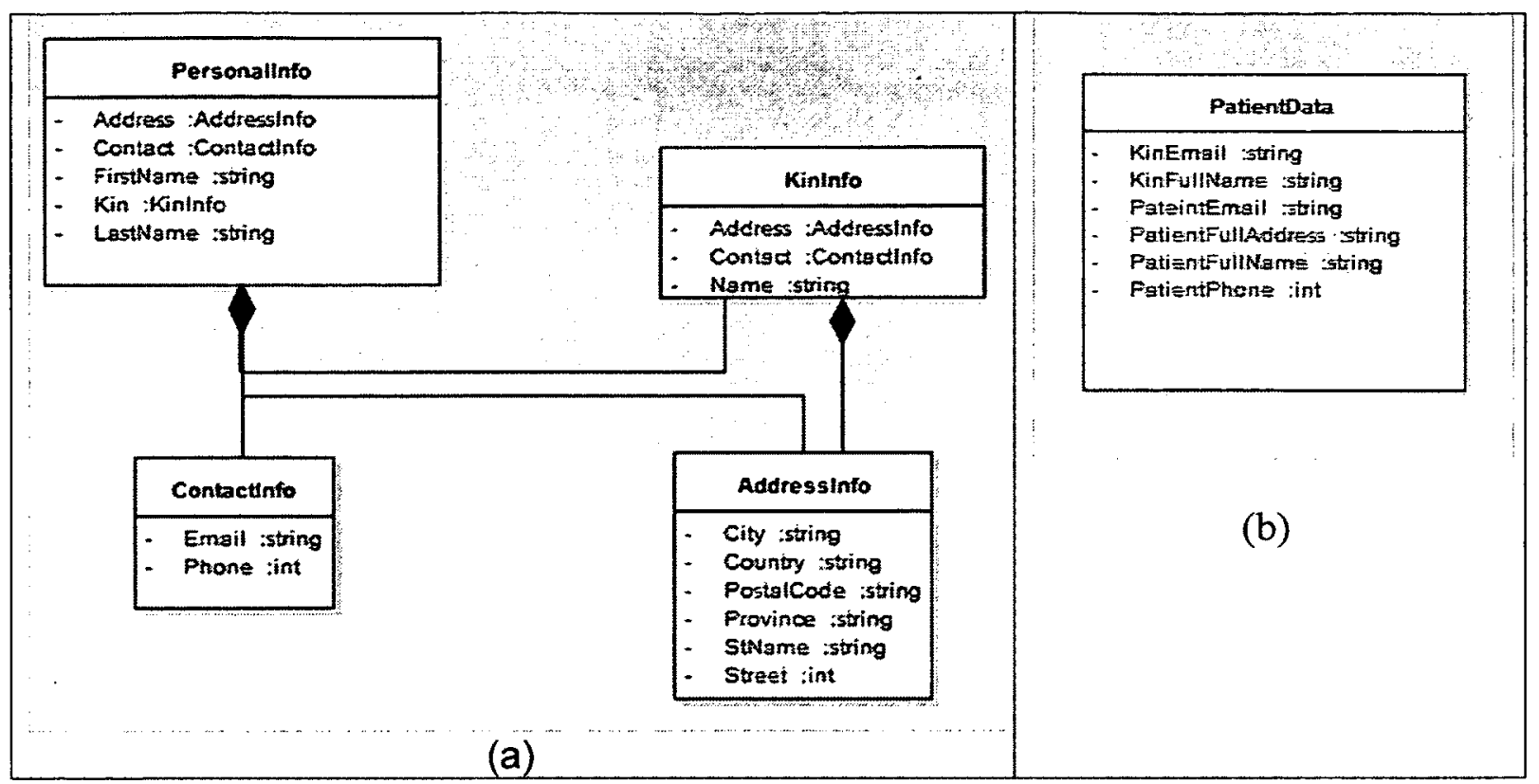

Figure 26 (a) Patient Data Representation in Emergency Client (b) Logically equivalent but simplified representation

\subsubsection{MESSAGE TRANSLATOR PERFORMANCE MEASUREMENTS}

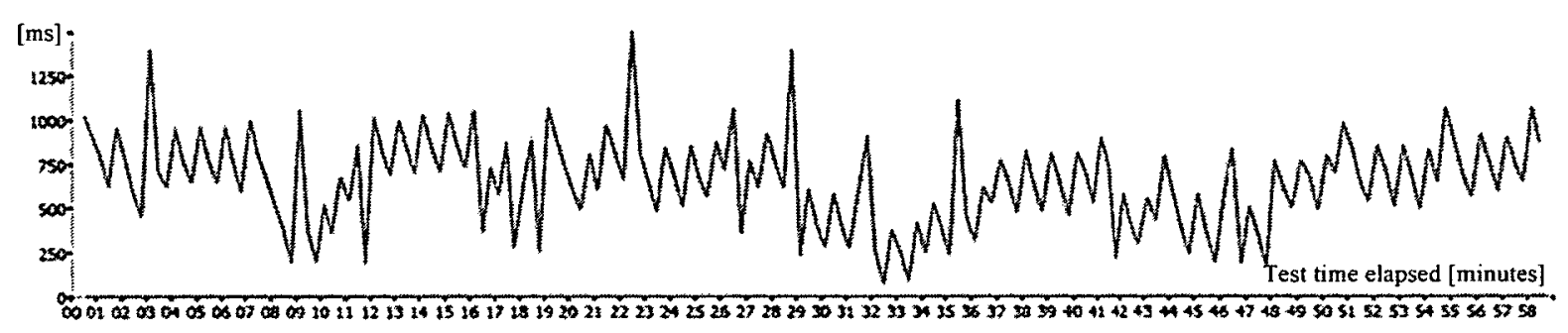

Figure 27 Message Translator Implementation Response Times

Table 12 Average Response Time - Message Translator

\begin{tabular}{|l|c|}
\hline Operation (end to end) & Average Response Time (ms) \\
\hline Message Translator & 669.10 \\
\hline
\end{tabular}

The Message Translator pattern is XML processing intensive. At minimum, it does the parsing of source XML document, XSLT transformation and creation of a new destination XML document. Any other processing due to business logic will be extra such as string concatenation, type casting and alike. 


\subsection{AGGREGATOR - PARALLEL CONVOY}

\subsubsection{PROBLEM}

Messages may be arriving in parts from one or multiple sources that need to be integrated together to form a single consolidated message. There is no pre-defined order of message arrival and ordered delivery is not important.

\subsubsection{SOLUTION}

The function of a parallel convoy (Hohpe and Woolf, 2003) is to collect and store individual messages required by a workflow until the complete set of related messages has been received. Then, the parallel convoy creates a single message from the individual input messages. The Aggregator achieves this by using the correlation identifier described in previous chapter 3 .

In the Parallel Convoy the arriving order for messages does not matter but further processing can only continue after all messages have been received. The message that arrives first initializes the correlation set (described in section 3.5) and the messages arriving after it will use it. The Parallel Convoy is applied when ordered delivery of messages is not important.

\subsubsection{APPLICATION}

In our case study, we have done the base implementation by using both the parallel convoy and the sequential convoy (presented in section 4.4) to test and evaluate the performance. As mentioned in the Correlation Identifier (section 3.5), the arriving messages related to patient may originate from various sources. The sequential or parallel convoy implementation does the integration of these incoming messages using the correlation identifier. Figure 28 depicts the steps of the Parallel Convoy pattern 


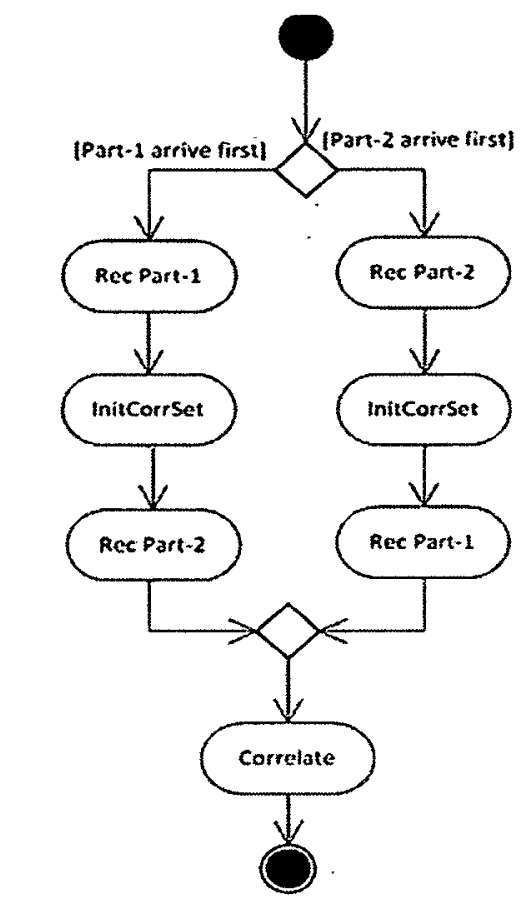

Figure 28 Parallel Convoy Pattern

As for the parallel convoy pattern implementation with Biztalk, it does not assume that the arrival of different message parts arrive in any given order; any message arriving first can initialize the workflow. Also the parallel mechanism is to make workflow implementation flexible in the sense that messages can be received in order, however it does not create threads in parallel for receiving or processing of messages.

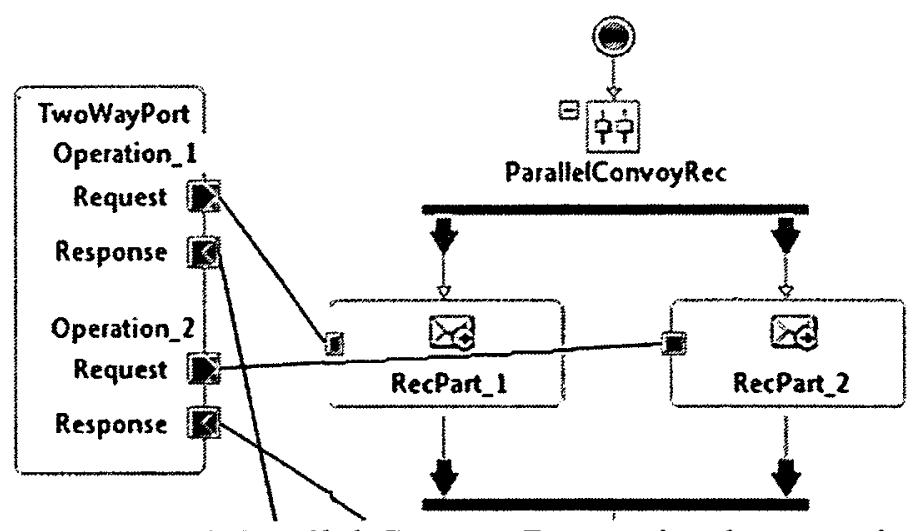

Figure 29 Parallel Convoy Pattern implementation in Biztalk 
The application of the use case will employ the usage of parallel convoy pattern as mentioned in Fig 28 and its realization in Biztalk environment is shown in Fig 29. The parallel convoy patterns itself is not indicative of the invocation mechanism or time required for invocation. However for response time measurement of our use case, we need to include the invocation time as the overhead of pattern cannot be measured without workflow invocation therefore in section 4.3 .4 , we can deduce the time consumed in parallel convoy pattern by utilizing the overall response time and knowledge gained from Table 14 obtained from profiling tool but yet it includes the workflow invocation and response time to client. The overall response time includes other delays such as, wait during hospital service call, creating and parsing SOAP envelope and sending the response back to requesting client which are not part of the pattern. The actual implementation of our use case utilizing parallel convoy pattern is shown in Figure 30 , for which the actual measurements have been performed and we will use these measurements to estimate the time consumed in the pattern itself.

It is interesting to note that the "fork" bar shown in Figure 29 and Figure 30 does not mean that the two activities, RecPart-1 and RecPart_2 are actually done in parallel. The Biztalk implementation of "fork" always attempts to execute the first branch, if possible, then the second. In the case the first message has not arrived yet, Biztalk cannot execute the first branch, so it will attempt to receive the second message if it is already there, then will wait for the other one. 


\subsubsection{PARALLEL CONVOY MEASUREMENTS}

The case study implementation given in Fig 30 applies the Parallel Convoy pattern at the beginning of the workflow. There are two steps in Fig. 30 that do not belong to the Parallel Convoy pattern and will be subtracted later, Send_Hospital and Rec-

Confirmation. Note that there are two message parts, each delivered in an un-defined order, which means that during our tests Message- 2 can arrive before Message- 1 or vice versa.

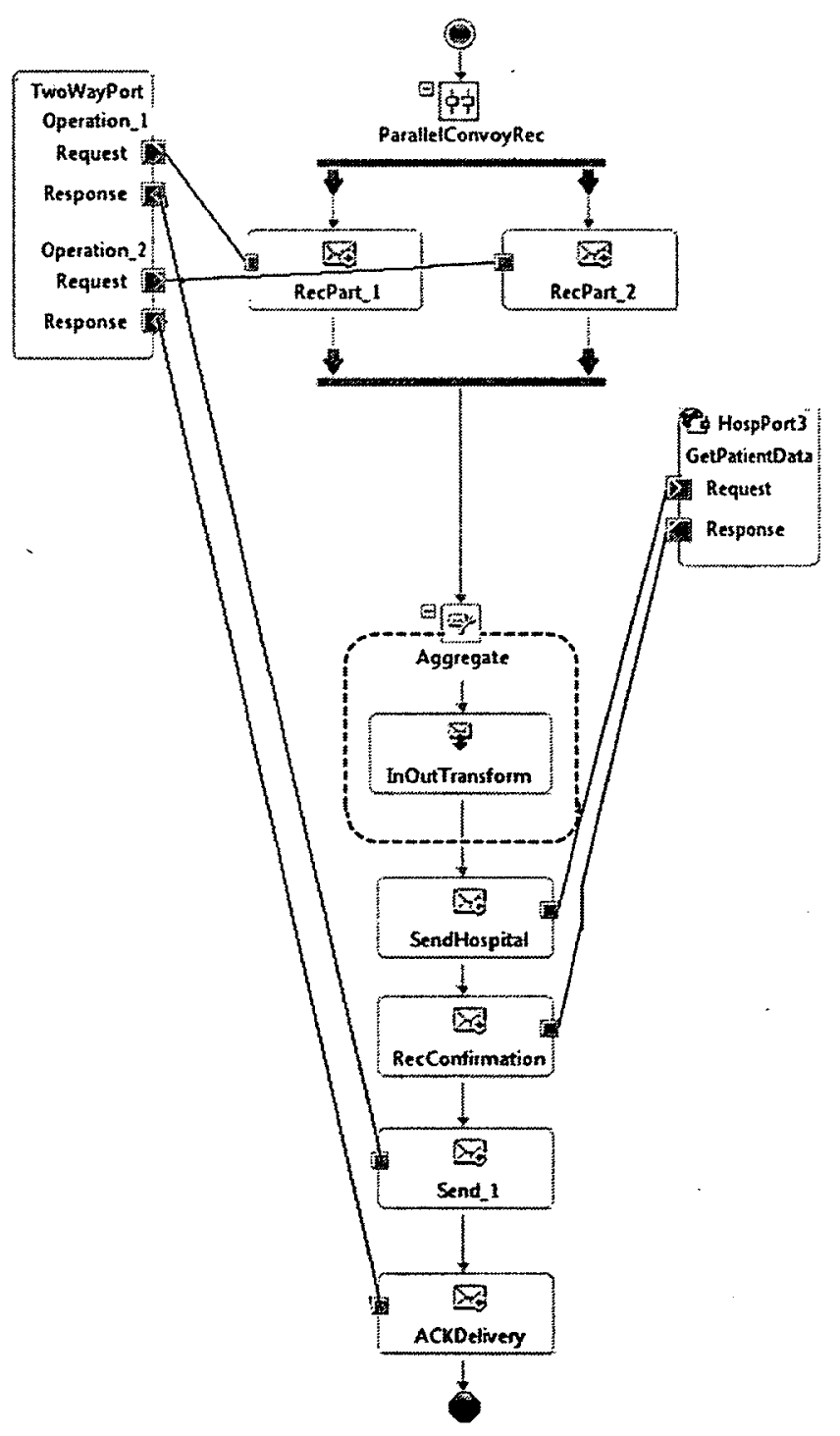

Figure 30 Parallel Convoy application in Biztalk to use case 
We measure the response time for each message separately which includes everything from the invocation from client side to sending a response back to client by the workflow for each message individually. There is a very small difference between the two response times in Table 13 so we can use one of them for further analysis, in order to exclude the workflow steps not directly related to the parallel convoy pattern.

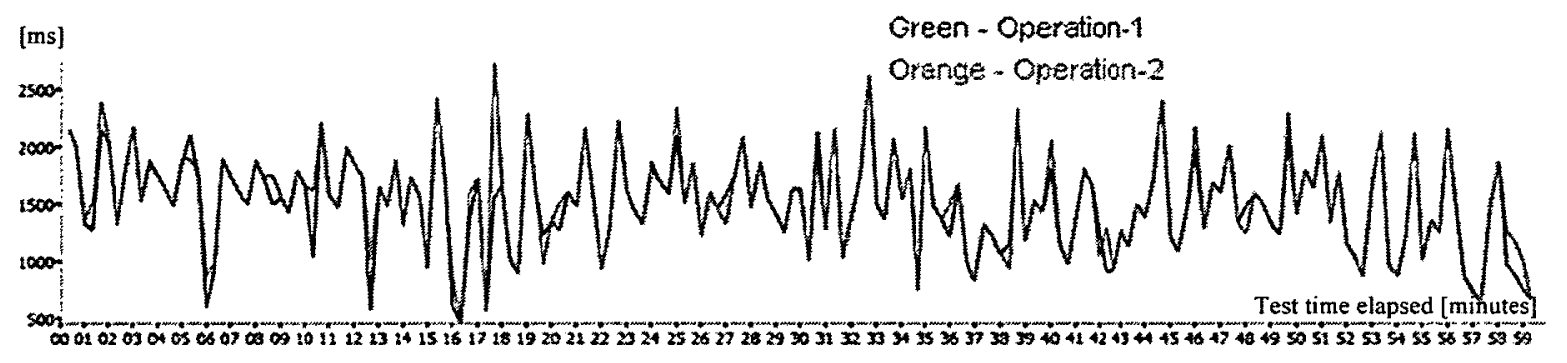

Figure 31 Parallel Convoy Application Response Times

Table 13 Average Response Time - Parallel Convoy application for use case

\begin{tabular}{|l|c|}
\hline Operation (end to end) & $\begin{array}{c}\text { Average Response } \\
\text { Time (ms) }\end{array}$ \\
\hline Message Part - 1 & 1297.74 \\
\hline Message Part - 2 & 1293.49 \\
\hline
\end{tabular}

Since the measuring tool allowed us to measure only end-to-end response times, from the arrival of a message on an input port (in this case, the invocation of Operation_1) until the corresponding reply is received (i.e., the return after the operation has ended), we have measured the response time for the two operations separately (see the results in Figure 30 and Table 13). Biztalk allows for the arrival of multiple messages on individual operations for the same port (in this case, Operation_1 and Operation_2 of the Two-way port shown in Figure 29). Also the measurements result includes a response message to client which is of the same size as the in-bound message. 
According to the approach from Figure 23, we used the orchestration profiler tool to get a more detailed assessment of the time required to complete each step in the workflow. Table 14 gives the results for the workflow steps.

Table 14 Detailed Assessment at each level of Parallel Convoy

\begin{tabular}{|c|c|c|c|c|c|c|}
\hline Shape Id Shape Name & Entry Count & Exit Count & $\begin{array}{l}\text { Success } \\
\text { Rate }(\%)\end{array}$ & $\begin{array}{l}\text { Minourat } \\
\text { ion (ms) }\end{array}$ & $\begin{array}{l}\text { MaxDura } \\
\text { tion (ms) }\end{array}$ & $\begin{array}{l}\text { AvgDurat } \\
\text { ion (ms) }\end{array}$ \\
\hline 1 RecPart_1 & 180 & 180 & 100 & 0 & 3 & 0 \\
\hline 4 Aggregate & 180 & 180 & 100 & 0 & 3 & 0 \\
\hline 5 RecPart_2 & 181 & 180 & 100 & 0 & 253 & 1 \\
\hline 6 SendHospital & 180 & $180^{\circ}$ & 100 & 16 & 523 & 43 \\
\hline 7 RecConfirmation & 180 & 180 & 100 & 150 & 1250 & 491 \\
\hline 8 Send_1 & 180 & 180 & 100 & 20 & 400 & 44 \\
\hline 9 ACKDelivery & 180 & 180 & 100 & 20 & 440 & 28 \\
\hline
\end{tabular}

As indicated in the table, the longest average time is at RecConfirmation, which is when the orchestration calls the hospital service and waits for a response. While waiting for the reply from the hospital service, the orchestration instance gets de-hydrated and it has to be re-hydrated when the web service response is received. However, the RecConfirmation step is not part of the parallel Convoy, so it will be subtracted from the measurement.

Another point where re-hydration may appear is before receiving the second part of the message. As seen in the table, the max value of RecPart2 $(253 \mathrm{~ms})$ is much larger than the max value of receive part 1 (only $3 \mathrm{~ms}$ ) summarized in Table 15 .

Table 15 Maximum delay associated with Parallel Convoy Receive parts

\begin{tabular}{|l|l|}
\hline RecPart_1 delay (max) & $3 \mathrm{msec}$ \\
\hline RecPart_2 delay (max) & $253 \mathrm{msec}$ \\
\hline
\end{tabular}


This is due to the fact that in some cases the second message of the convoy arrives late and the orchestration has to be re-hydrated upon its reception. However, this does not happen really often as suggested by the average value.

The overhead involved in the parallel convoy pattern application shown in Fig 30 is hidden behind the scene and is due to Correlation, De-Hydration and Re-Hydration and subscription resolution. This overhead is part of the parallel convoy pattern included in overall response time of both operations as seen by the client, but its assessment is not explicitly included in Table 14. We can estimate this hidden overhead by subtracting the other workflow steps namely SendHospital and RecConfirmation. This non-pattern related steps are mainly responsible for the invocation of hospital web service, which causes the orchestration instance to de-hydrate again while waiting for response from web service and the to re-hydrate the instance when response is received.

Parallel convoy overhead $\approx$ Response Time (Operation1)-Non-pattern-steps

$$
=1297.75-(43+491)=763.75
$$

The $763.75 \mathrm{msec}$ delay is mainly due to the new workflow instance creation, publishsubscribe mechanism for message delivery to the appropriate workflow, reliable messaging to persist every into MessageBox DB and de-hydration and re-hydration, (which are due to the workflow instance being persisted to database while waiting for other message part to arrive). It also includes the delay due to the workflow service invocation and sending its response back to client. We will denote the hidden Parallel Convoy overhead that appears when this pattern is applied at the beginning of the workflow as Initialization Delay D1 in subsequent sections. The D1 delay is a considerable hidden overhead of which application designers should be aware. . 


\subsection{AGGREGATOR - SEQUENTIAL CONVOY}

\subsubsection{PROBLEM}

Messages may be arriving in parts from one or multiple sources that need to be integrated together to form a single consolidated message. There is a pre-defined order of message arrival and ordered delivery is important.

\subsubsection{SOLUTION}

In the Sequential Convoy (Hohpe and Woolf, 2003) the messages are received in a predefined ordered sequence. The first message initializes the correlation set while the other messages use it. A correlation set is a data unique to a message or a set of messages that can be used to tie together the messages that needs to be processed together by a particular process instance. The sequential convoy variant is applied when the ordered delivery of messages is important.

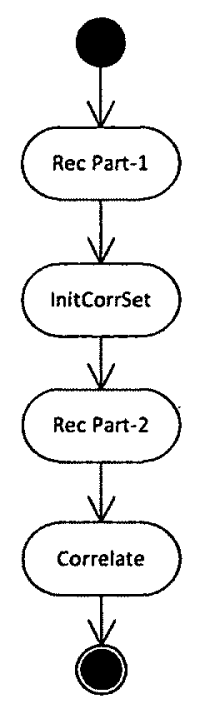

Figure 32 Sequential Convoy Pattern 


\subsubsection{APPLICATION}

In our case study, we have done the base implementation by using both types of convoys to test and evaluate their performance. As mentioned in the Correlation Identifier, the arriving messages related to patient may originate from various sources. The sequential or parallel convoy implementation does the integration of these incoming messages using the correlation identifier. In this implementation we make use of Sequential Convoy.

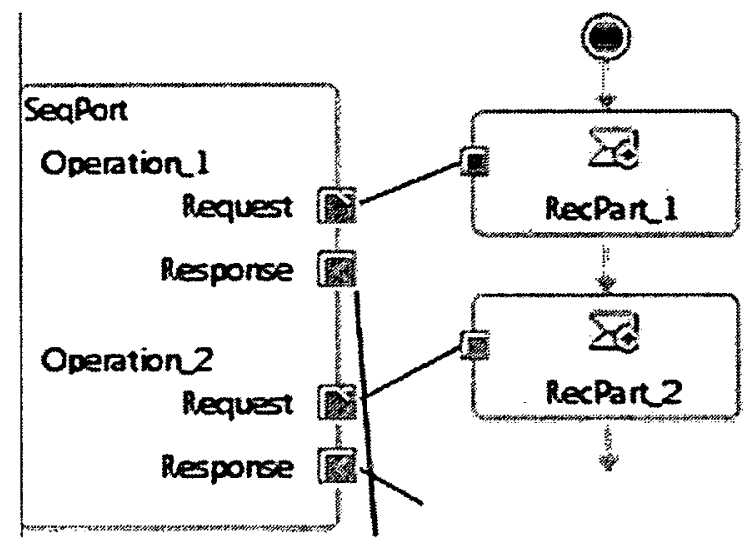

Figure 33 Sequential Convoy pattern application in Biztalk

Figure 32 shows the sequential convoy pattern and Figure 33 shows its realization in Biztalk which also shows the ports used for receiving messages and sending response back to client, these ports are not part of the pattern. The application of use case above employ the usage of sequential convoy pattern, therefore in next section we can deduce the time consumes in sequential convoy pattern only by utilizing the overall response time and knowledge gained from Table 17 obtained by profiling tool. 


\subsubsection{SEQUENTIAL CONVOY PERFORMANCE MEASUREMENTS}

Similar to parallel convoy, the messages can arrive in any order, however in this case if Message-2 arrives first it is queued in MessageBox DB and orchestration instance is not created until Message-1 arrives to initialize the correlation set.

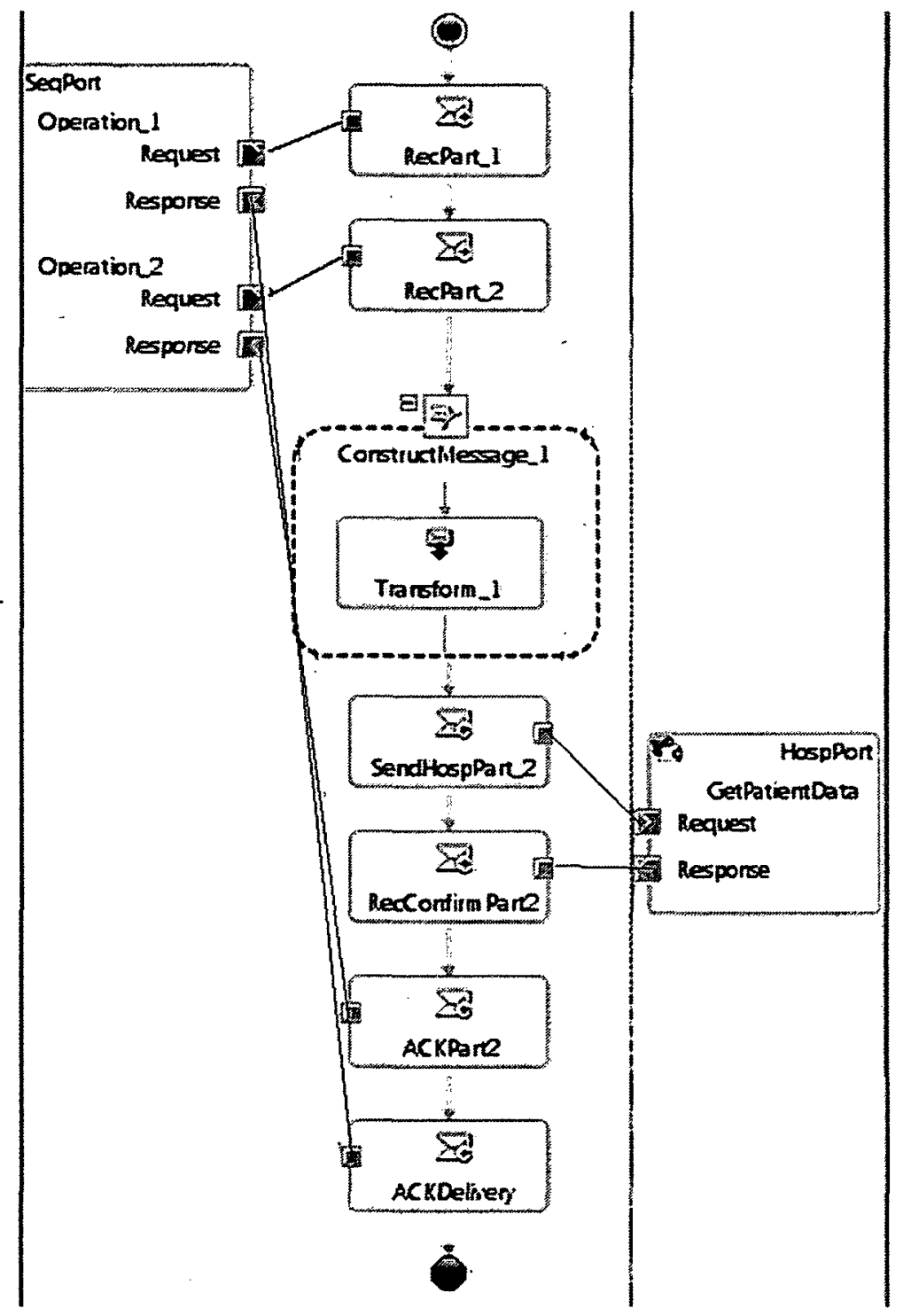

Figure 34 Sequential Convoy application in Biztalk to use case 
The response time includes the time from invocation of workflow by client until a response from workflow is received by client as shown in Fig 34 . This includes overhead other than pattern itself as in Table 17 and cannot be measured separately given the measurement tools limitations.

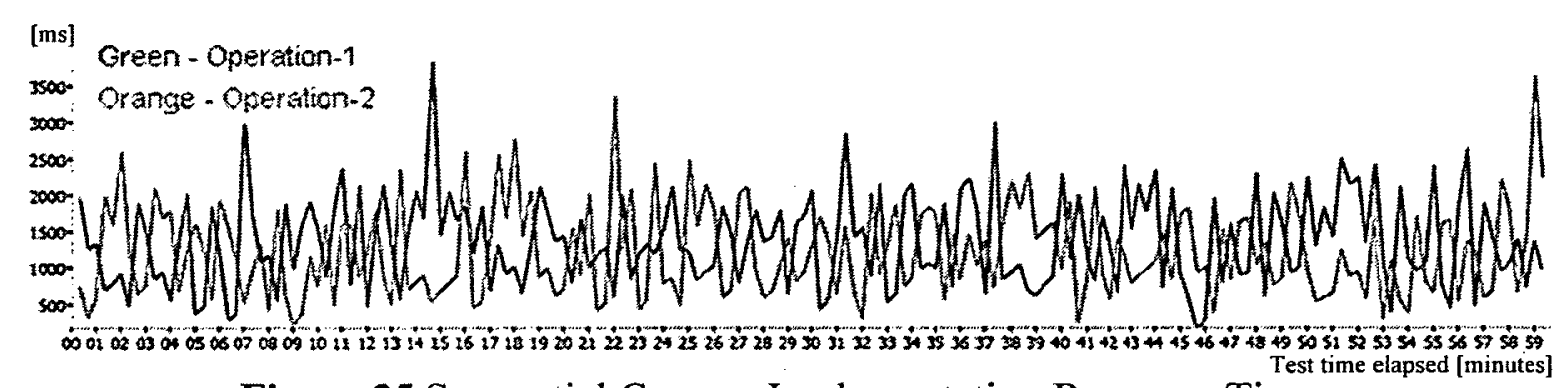

Figure 35 Sequential Convoy Implementation Response Times

Table 16 Average Response Time - Sequential Convoy application to use case

\begin{tabular}{|l|c|}
\hline $\begin{array}{c}\text { Operation (end } \\
\text { to end) }\end{array}$ & $\begin{array}{c}\text { Average Response Time } \\
\text { (ms) }\end{array}$ \\
\hline Message Part - 1 & 1331.74 \\
\hline Message Part - 2 & 1364.06 \\
\hline
\end{tabular}

Table 17 Detailed Assessment at each level of Sequential Convoy

\begin{tabular}{|c|c|c|c|c|c|c|}
\hline Shape Id Shape Name & Entry Count & Exit Count & $\begin{array}{l}\text { Success } \\
\text { Rate (3o) }\end{array}$ & $\begin{array}{l}\text { MinDurat } \\
\text { ion (ms) }\end{array}$ & $\begin{array}{l}\text { MaxDura } \\
\text { zion (ms) }\end{array}$ & $\begin{array}{l}\text { AvgDurat } \\
\text { ion (ms) }\end{array}$ \\
\hline 1 Construckmessage_1 & 180 & 180 & 100 & 0 & 3 & 0 \\
\hline 2 RecConfirmpart 2 & 180 & 180 & 100 & 170 & 1326 & 555 \\
\hline 3 RecPart_2 & 180 & 180 & 100 & 0 & 253 & 5 \\
\hline 4 ACKDelivery & 180 & 180 & 100 & 0 & 30 & 4 \\
\hline 5 ACKPart2 & 180 & 180 & 100 & 23 & 526 & 46 \\
\hline 6 RecPar__1 & 180 & 180 & 100 & 0 & 63 & 0 \\
\hline 8 SendHosppart_2 & 180 & 180 & 100 & 16 & 806 & 67 \\
\hline
\end{tabular}


The sequential convoy is subject to same de-hydration and re-hydration overheads as parallel convoy with the exception that only message part- 1 can create a workflow instance and initialize the correlation set but it doesn't mean that part-1 always arrives before its counterpart as this is dictated by clients sending messages over which the orchestration has not control. In this case Message-2 is queued in MessageBox DB and wait for Message-1 to arrive to create new orchestration instance.

In many instances in our 180 times invocations, part 2 arrives before part 1 in which case part2 is persisted into a repository until part1 arrives to initialize orchestration instance and when that happens, part2 needs to be de-queued from the repository and delivered to orchestration instance. This de-queuing of message part2 dictates the fact that average response time of sequential convoy is slightly more than that of a parallel convoy.

Similar to parallel convoy, the overhead involved in sequential convoy application shown in Fig 30 is hidden behind the scene and is due to Correlation, De-Hydration and ReHydration and subscription resolution. This overhead is part of overall response time of both operations as seen by the client but the assessment is not included in Table 17. There is non-sequential convoy related overhead namely ConstructMessage, SendHospPart2, RecConformPart2 which is included in response time of each of both messages, therefore it must be excluded from overall response time to estimate pattern related overhead only. We can estimate the delay due to the sequential convoy as follows:

Seq. convoy overhead $\approx$ Response Time Operation 1 - Not pattern Steps

$$
=1331.743-(555+67)=709.74
$$

The seq. convoy overhead still includes delay due to workflow invocation and sending response back to client. 


\subsection{CONTENT BASED ROUTER}

\subsubsection{PROBLEM}

Determine the destination of out-bound message dynamically from the in-bound message.

\subsubsection{SOLUTION}

Content based routing (Hohpe and Woolf, 2003) in an SOA system occurs when destination of an outbound message is dependent on or determined from information contained in inbound message.

Figure 36 Content Based Router Pattern
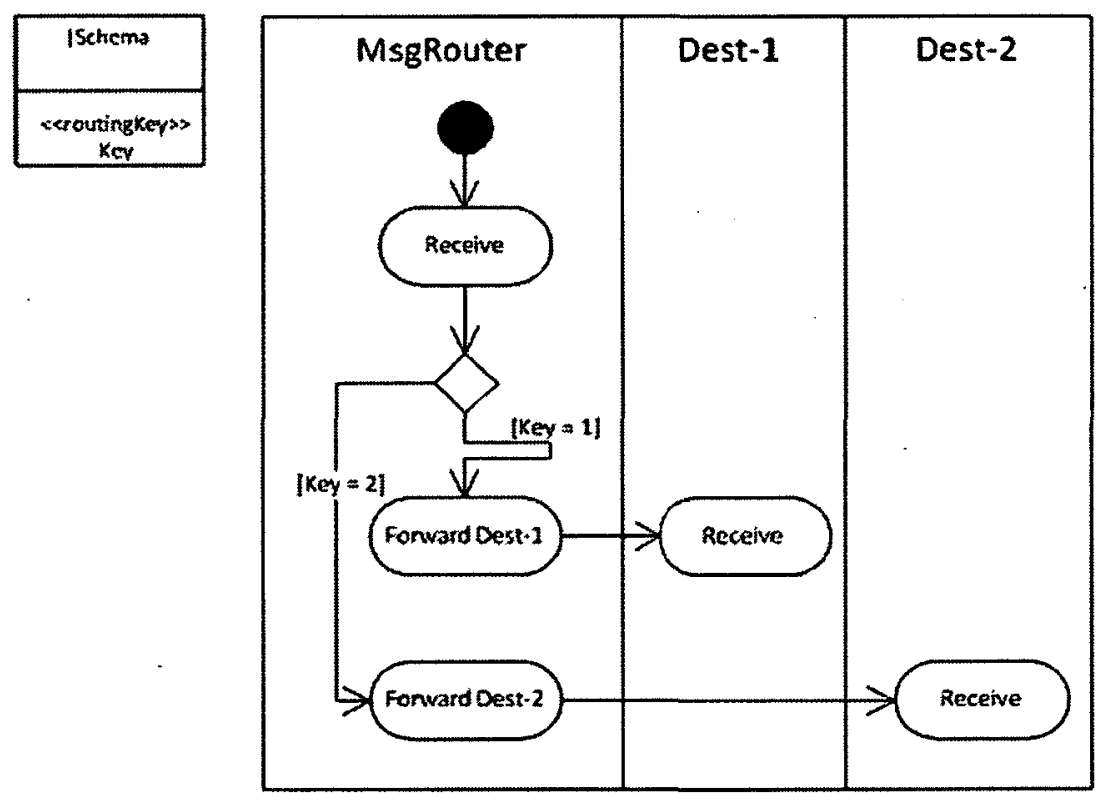

\subsubsection{APPLICATION}

Figure 37 shows the implementation of Content Based Router pattern in Biztalk. The destination hospital URL is contained within the message arriving from requesting client. The pattern parses the message, extracts the URL and forwards it to intended destination. In our case study, we also use this pattern, to improve reducing response time. 
Figure 37 Biztalk Implementation of Content Based Router

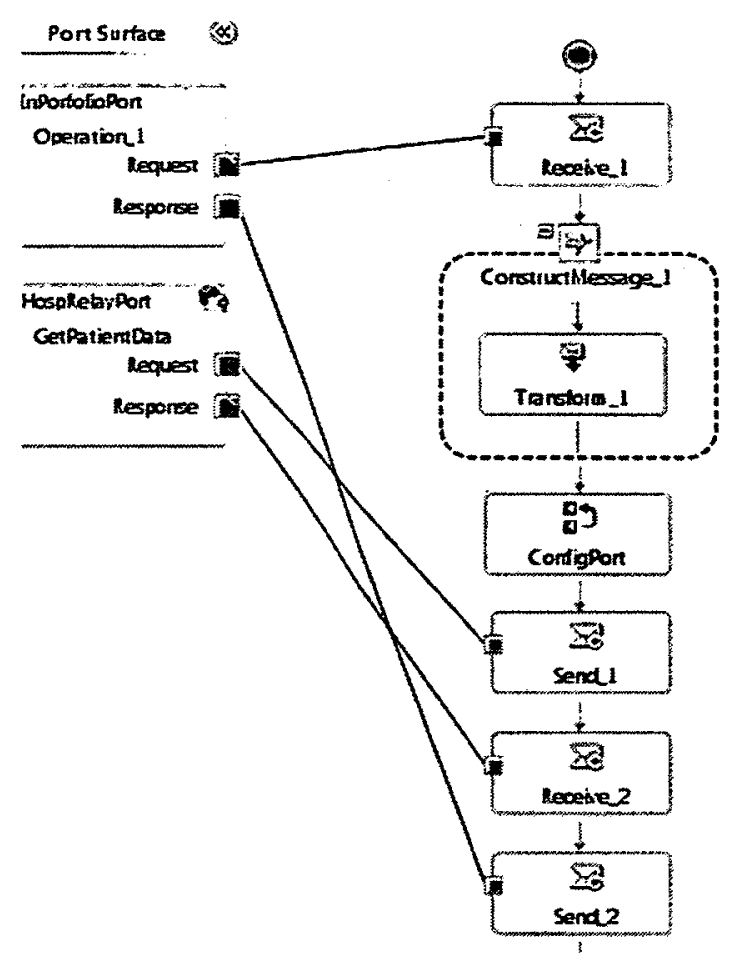

In a basic implementation the emergency site will send its address or postal code to the workflow system, which is then responsible to determine the closest hospital using the address information in the inbound message and calling a third party web service. In content based routing system, we make the client implementation more complex by having it to determine the required hospital and providing their web service URL as part of the inbound message.

\subsubsection{CONTENT BASED ROUTER MEASUREMENTS}

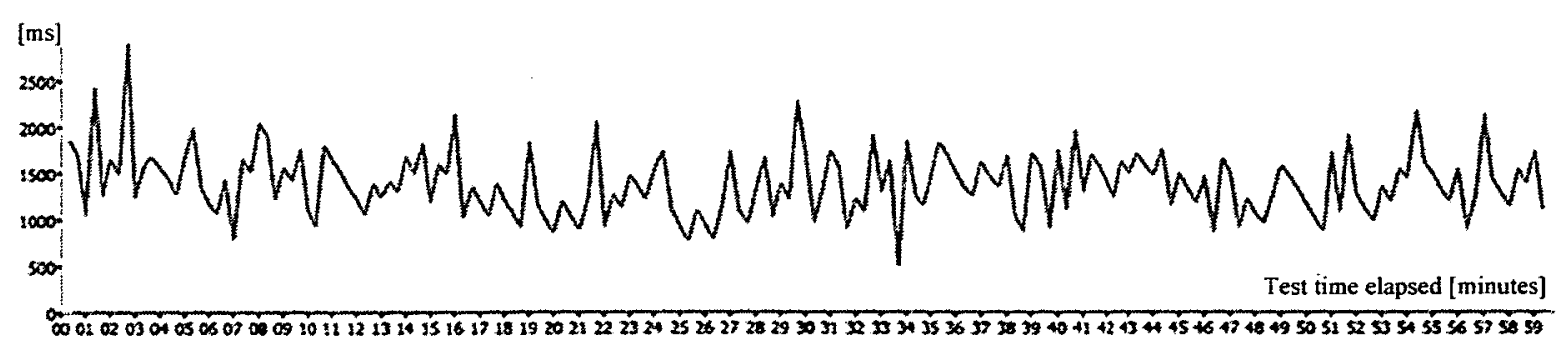

Figure 38 Content Based Router Implementation Response Times 
Table 18 Average Response Time - Content Based Router

\begin{tabular}{|l|c|}
\hline Operation (end to end) & Average Response Time (ms) \\
\hline Client Aggregated Message & 1080.21 \\
\hline
\end{tabular}

Table 19 Detailed Assessment at each level of Content Based Router

\begin{tabular}{|c|c|c|c|c|c|c|c|}
\hline Shape Id & Shape Name & $\begin{array}{l}\text { Entry } \\
\text { Count }\end{array}$ & $\begin{array}{l}\text { Exit } \\
\text { Count }\end{array}$ & $\begin{array}{l}\text { Success } \\
\text { Rate (\%) }\end{array}$ & $\begin{array}{l}\text { MinDurat } \\
\text { inon (ms) }\end{array}$ & $\begin{array}{l}\text { MaxDura } \\
\text { tion (ms) }\end{array}$ & $\begin{array}{l}\text { AvgDurat } \\
\text { ion (ms) }\end{array}$ \\
\hline \multicolumn{2}{|c|}{1 Send_2 } & 180 & 180 & 100 & 6 & 190 & 27 \\
\hline \multicolumn{2}{|c|}{2 ConstructMessage_1 } & 180 & 180 & 100 & 0 & 13 & 0 \\
\hline \multicolumn{2}{|c|}{3 Receive_2 } & 180 & 180 & 100 & 126 & 1370 & 535 \\
\hline \multicolumn{2}{|c|}{ 4: Receive_1 } & 180 & 180 & 100 & 0 & 63 & 0 \\
\hline \multicolumn{2}{|c|}{6 Send_1 } & 180 & 180 & 100 & 20 & 266 & 55 \\
\hline \multicolumn{2}{|c|}{7 Expression_1 } & 180 & 180 & 100 & 0 & 3 & 0 \\
\hline
\end{tabular}

As from Table 18, the response time of the design based on the content based router pattern is less than that of the parallel and sequential convoy. This is because it avoids receiving an extra message which saves the application of hidden platform patterns overheads related to that message. Also there is no need of correlation of messages to be performed as there is only one single message.

However, in this case it becomes the client's responsibility to integrate all the relevant information and send it as a single message. There is no de-hydration and re-hydration of orchestration instance performed as the orchestration can continue its normal execution after the reception of the single message. 


\subsection{SCATTER AND GATHER}

\subsubsection{PROBLEM}

A business process may require decision making after soliciting responses from various third parties and then selecting the response from a particular party based on a certain policy.

\subsubsection{SOLUTION}

Scatter and Gather pattern (Hohpe and Woolf, 2003) in a business process is used when a business process solicits response from various parties regarding a particular problem and then makes decision as defined by the business rule. Figure 39 shows steps involved in Scatter and Gather pattern. In a Biztalk implementation of this pattern, this also introduces the usage of Business Rules Engine (BRE). The BRE allows changing the rules or policy as it becomes necessary with minimum interruption to a running system.

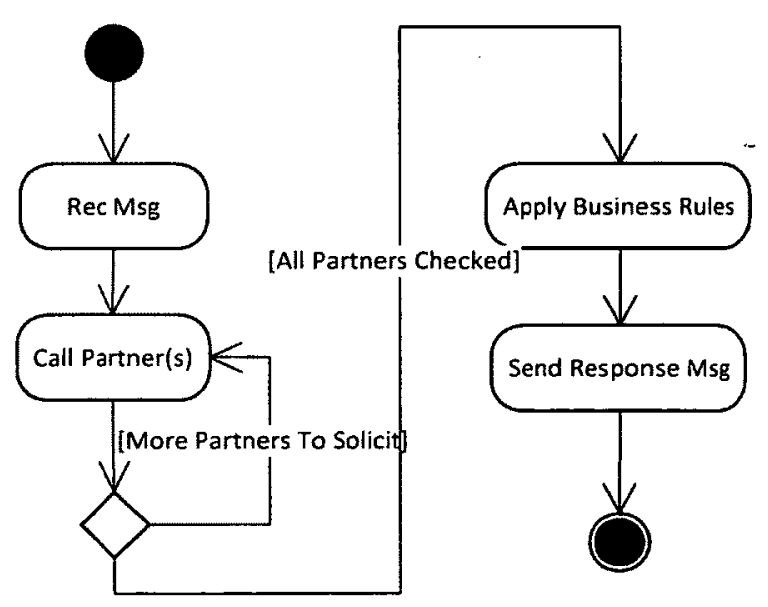

Figure 39 Scatter and Gather Pattern

The introduction of Business Rules Engine elevates the need of re-compile and redeployment of a running system in case the rules change. 


\subsubsection{APPLICATION}

In one use of our case study, before sending information to hospital, we solicit each emergency care provider facility to provide existing patient load in emergency room. This is particularly useful in appropriate load balancing of patients among hospitals. For example, less critical or non-life threatening cases may be dispatched to distant hospitals if they have adequate vacancy.

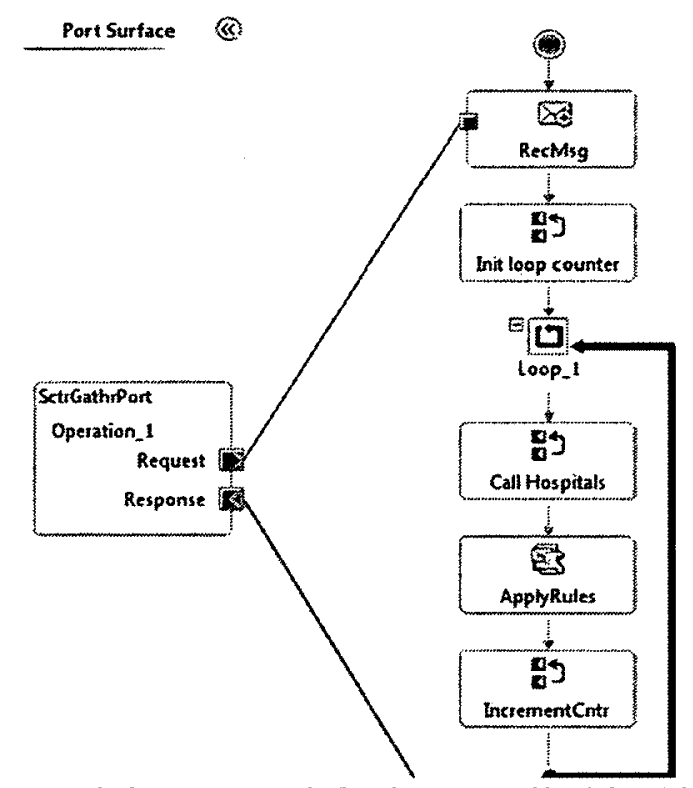

Figure 40 Scatter and Gather Applied in Biztalk

\subsubsection{SCATTER AND GATHER MEASUREMENTS}

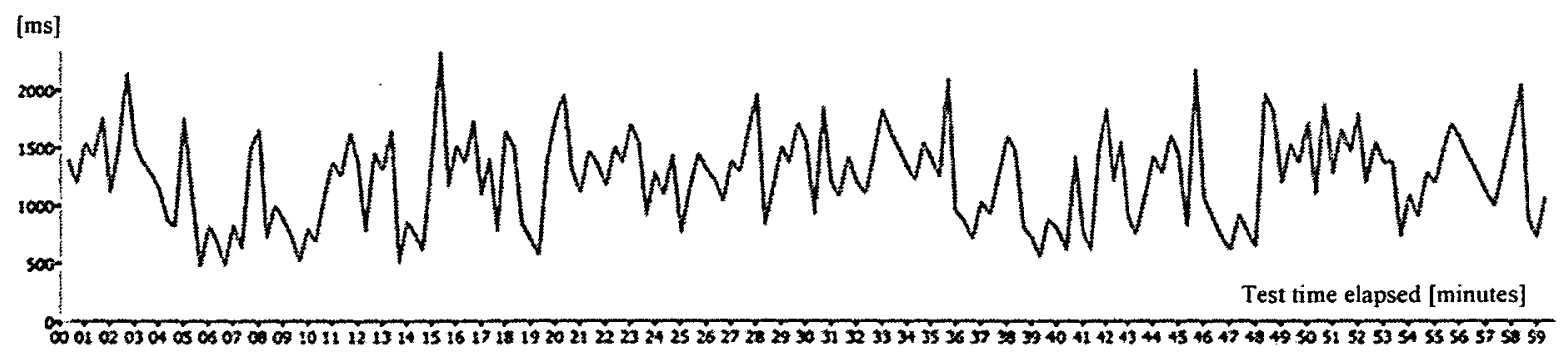

Figure 41 Scatter and Gather Implementation Response Times 
Table 20 Average Response Time - Scatter and Gather

\begin{tabular}{|l|c|}
\hline Operation (end to end) & Average Response Time (ms) \\
\hline Client Aggregated Message & 1154.89 \\
\hline
\end{tabular}

Table 21 Detailed Assessment at each level of Scatter and Gather

\begin{tabular}{|c|l|l|l|r|l|l|l|}
\hline Shape Id & Shape Name & $\begin{array}{l}\text { Entry } \\
\text { Count }\end{array}$ & $\begin{array}{l}\text { Exit } \\
\text { Count }\end{array}$ & $\begin{array}{l}\text { Success } \\
\text { Rate (\%) }\end{array}$ & $\begin{array}{l}\text { MinDuration } \\
(\mathrm{ms})\end{array}$ & $\begin{array}{l}\text { MaxDuration } \\
\text { (ms) }\end{array}$ & $\begin{array}{l}\text { AvgDuration } \\
\text { (ms) }\end{array}$ \\
1 & CheckDistance & 560 & 560 & 100 & 0 & 1276 & \\
2 & IncrementCntr & 507 & 507 & 100 & 0 & 0 \\
3 & SendHospUrl & 184 & 184 & 100 & 23 & 960 & 0 \\
5 & RecMsg & 184 & 184 & 100 & 0 & 126 & 45 \\
7 & ConstructMessage_1 & 184 & 184 & 100 & 0 & 16 & 0 \\
9 & Send_1 & 181 & 181 & 100 & 0 & 33 & 3 \\
10 & Expression_1 & 184 & 184 & 100 & 0 & 3 & 0 \\
11 & Init loop counter & 184 & 184 & 100 & 0 & 0 & 0 \\
12 & Confirmation & 184 & 184 & 100 & 150 & 1730 & 514 \\
\hline
\end{tabular}

From the table above, it is apparent that major delay is involved in the Confirmation step which is when a web service response is received and the orchestration instance is rehydrated again from its de-hydrated state. Other less significant delay is due to CheckDistance as this step invokes the Biztalk Business Rules engine for decision making.

\subsection{ORCHESTRATION INSTANCE CREATION AND RESTORATION}

AFTER LONG WAIT

In the above convoy implementations and from the analysis of results, we can see that there is a Initialization Delay D1 of about $763.75 \mathrm{~ms}$ as indicated in section 4.3.4, which is not reported explicitly by the Orchestration profiling tool. It is due to the fact that this overhead occurs before any orchestration instance is created. Prior to the creation of the orchestration instance, the message arrives at the end-point, the context for the promoted 
properties is created and attached to message, which is then persisted into the message box together with the attached context. The Biztalk subscription mechanism dequeues the messages from the message box and takes care of initializing or rehydrating the appropriate orchestration instance. This delay implied by this process is not in captured by the profiling tool, but can be seen as the difference between the end-to-end response time and the sum of the step averages provided by the profiling tool. We denote this delay by $D 1$. This delay can be verified by the measurement from the previous chapters used to invoke an empty workflow service in order to evaluate the overhead due to the platform-provided patterns in which case there is no correlation and re-hydration performed due to fact that there is only one message but still there other overhead is involved due to creation of property schema, persistence to message box, subscription resolution and new orchestration instance creation.

At the development level, we can observe that the multiple receive steps, such as in convoys, increases the overall response time due to the processing of the message correlation set and re-hydration of orchestrations in the cases when the subsequent messages are arriving later than the previous message.

Another common delay element in all implementations is the delay caused by the dehydration and rehydration process when a third party web service is invoked, similar to the hospital web service in our case study. The delay to rehydrate an orchestration after receiving a response from a web service is about $500 \mathrm{~ms}$, which also includes the overhead of invoking and receiving response from a simple SOAP service, which is about 
$10 \mathrm{~ms}$, as measured in previous chapter. According to this, we can assume that the overhead required for the dehydration and rehydration of an orchestration instance takes on average $490 \mathrm{~ms}$ on the system configurations we used for measurements. Let us denote the dehydration and rehydration overhead by $D 2$ (in ms). Therefore, if there are $N$ Web Service invocations in an orchestration, the likely overhead for dehydration and rehydration will be in order of $N^{*} D 2 \mathrm{~ms}$.

Similarly, if there are $M$ message parts that arrive initially to an invoked workflow service that need to be received by the workflow, the minimum average delay due to instance creation, publish-subscribe, reliable messaging, dehydration and rehydration can be order of $(M-1)^{*} D 1 \mathrm{~ms}$. Unfortunately, we could not investigate more closely this delay and its relationship with the number of message parts that need to be received with the measurement tools available in this research.

We can estimate that if there are $M$ messages parts arriving to an orchestration that need to be received and processed together for each invocation, and if this orchestration calls $N$ web services during its execution, the maximum average overhead due to dehydration and rehydration (which are hidden patterns) would be:

$$
((M-1) * D 1)+(N * D 2)
$$

\subsection{RESPONSE TIME MEASUREMENT CHALLENGES}

Since a broad range of patterns are implemented at platform level, it becomes difficult to measure effect of each step on end to end response time due to lack of instrumentation at fine grained levels. A detailed knowledge of platform implementation and API helps in 
determining what overheads are involved and to associate them to generic patters but measuring the performance cost tag attached to each stage is difficult such as time required to determine a message subscriptions (Publish-Subscribe patterns), to persist message to database (reliable messaging) and to de-hydrate and re-hydrate workflows. However, wherever possible techniques such as eliminating the known delay of steps in workflow from overall response time and incrementally building the system can help estimate delays like $D 1$ and $D 2$. 


\section{PREDICTIVE PERFORMANCE EVALUATION}

\subsection{INTRODUCTION}

In chapter 3 we evaluated the overhead that lies in patterns implemented by the development and hosting platform, whereas in chapter 4 we measured the performance implications of development patterns such as sequential and parallel convoy. We can combine the knowledge gained to predict the performance of a SOA application that is not implemented yet. This helps the designer of the workflows to gain a basic idea of the performance of the system being designed by using our approach to predict the performance overhead of the new system, then decide which patterns to use and why. We evaluate this process with a new case study, whose response time is estimated based on the design and our pattern measurements from Chapters 3 and 4 . Next we validate the estimations against measurements based on the actual implementation and execution of the system on top of Biztalk.

\subsection{CASE STUDY}

This case study provides an automated solution for handling the prescription renewal requests from a patient and determines the coverage of the drug by the insurance provider. It is a part of the Electronic Prescribing Workflow Analysis Handbook developed by the eHealthObservatory research team at the University of Victoria (Partridge and Bassi, 2011). As clinicians migrate to using electronic medical records (EMRs), one of the key workflow changes they will encounter will be the use of the computer in prescribing. The ePrescribe system provided by the eHealthObservatory has 5 stages that implements more automation steps using computer assisted workflows as 
ePrescribe process moves into the next stage, from 1 to 5 . In stage 5 , the pharmaceutical insurance coverage process is linked within the EMR as part of a region-wide ePrescribe system, where the request for prescription renewal, approval, and subsequent prescription generation are all part of the system.

We will implement the use case of stage-5 from (Partridge and Bassi, 2011), which deals with the prescription renewal request from the patient, evaluation of prescription coverage by the insurance and then finalizing the initial prescription or some alternate prescription, after receiving coverage details from insurance. The availability and administration of prescription is dependent on many factors, insurance coverage being one of them. The patient may or may not have valid insurance coverage; further certain insurance plan may not provide drug coverage or coverage for certain type of drugs. In this case the administering authority may decide to provide alternate drug which is less costly or covered by the insurance plan.

\subsection{DESIGN}

The administering authority workflow receives patient personal and prescription renewal request information in two separate messages, which it integrates into one single message and forwards it to the insurance company, by invoking a workflow service to evaluate the coverage. The insurance company workflow receives the message and invokes a web service to retrieve coverage plan details for the said patient and determine the extent of coverage. The web service checks if the patient's insurance plan is active or not, if yes then it checks if requested drug is covered or not and what percentage of cost is covered. 
The insurance workflow receives the web service response and forwards it back to the administering authority workflow. This design is shown in the following figure.
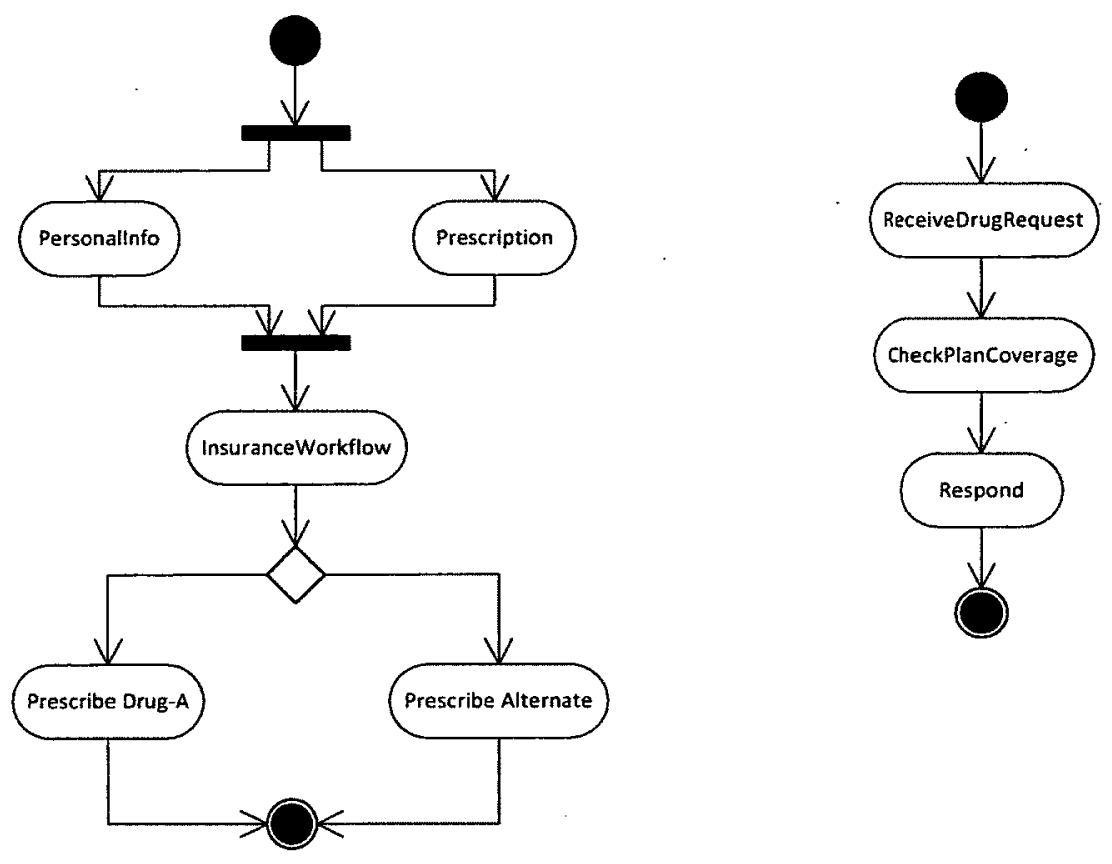

Figure 42 Prescription renewal workflow design (main workflow and insurance service)

Based on the analysis and workflow diagram, the designer has decided to apply the parallel convoy pattern as the messages from patient will be arriving randomly and need to be correlated for any given patient. Further he determines that the insurance service is also implemented as a workflow, which has the overhead of publish-subscribe, reliable messaging, correlation, de-hydration, re-hydration and pipeline invocation along with web service invocation that checks patient records.

\subsubsection{RESPONSE TIME ESTIMATION}

The designer can refer to the overhead measurements presented in last two chapters to estimate the average overhead involved in his design assuming that only one user invokes the workflow at any given time. 
One challenge is that the measurement results presented in Chapter 3 and 4 are dependent on the hardware and software configuration used for measurements. Other configuration may give different timing results, so we will assume that either: a) the measurements from chapter 3 and 4 were repeated on the new configuration to obtain the corresponding overhead values; or b) the designers applies a scaling factor (which is obtained from other experiments) between the two configurations. In any case, scaling measurement results from a configuration to another one is a complex problem that requires future investigation.

In order to estimate the overhead for a new design for the prescription refill workflow from the ePrescribe system, considered as a case study for this chapter, we refer to:

- Measurements results of Parallel Convoy pattern to check what overhead is involved.

- Measurements results for workflow invocations with variable data size (which in this case is $1 \mathrm{~KB})$.

Beside the above overheads, we realize that other delays will contribute to the workflow response time due to business logic and the execution of external services invoked. Assuming that in our case we will be dealing with messages with $1 \mathrm{~KB}$ data size, the minimum average overhead introduced by patterns in the prescription refill workflow is estimated in Table 22. 
Table 22 Patient prescription refill workflow response time estimates

\begin{tabular}{|l|c|}
\hline \multicolumn{1}{|c|}{ Delay Element } & Estimated average response time (ms) \\
\hline $\begin{array}{l}\text { Parallel convoy for two messages with 1 } \\
\text { section 4.7) }\end{array}$ & 763.75 \\
\hline $\begin{array}{l}\text { De-Hydration and Re-Hydration of main } \\
\text { workflow when child workflow is invoked } \\
\text { (It includes D2 factor from section 4.7) }\end{array}$ & 500 \\
\hline $\begin{array}{l}\text { Workflow invocation with a single } \\
\text { message with 1-10 KB of data }\end{array}$ & \\
\hline Invocation of a simple web service & 644.42 \\
\hline \multicolumn{1}{|c|}{ Total } & 8.54 \\
\hline
\end{tabular}

Based on above table, we determine that the average overhead due to patterns will be $1916.71 \mathrm{~ms}$ or approximately $2000 \mathrm{~ms}$. In a similar way, looking at the response time measurement in chapter 3 for variable message sizes, we can estimate the response time of the prescription refill workflow for different message sizes as an illustration of the fact the it possible to consider the message data size in the estimation. This will be demonstrated and compared with actual values later in this chapter.

It is important to note that a significant contribution to the overhead is due to the Biztalkprovided patterns involved in the workflow invocation and the parallel convoy. Such patterns are in fact hidden from the developer and their effect is easy to overlook if the developer does not have enough insight into the performance overhead brought by the service platform. The overhead due to business logic in this case is very small compared 
with the platform overhead. In cases where business logic is computation intensive such as multiple nested loops, data access, disk $\mathrm{I} / \mathrm{O}$, appropriate estimates need to be factored in; however they were not required in this case.

The next section describes the implementation of the workflow on top of Biztalk and the actual response time measurements.

\subsection{SYSTEM IMPLEMENTATION IN BIZTALK}

The insurance workflow which also invokes a simple SOAP web service to access patient coverage details is shown in Figure 43 whereas the main workflow called by the requesting client is shown in Figure 44.

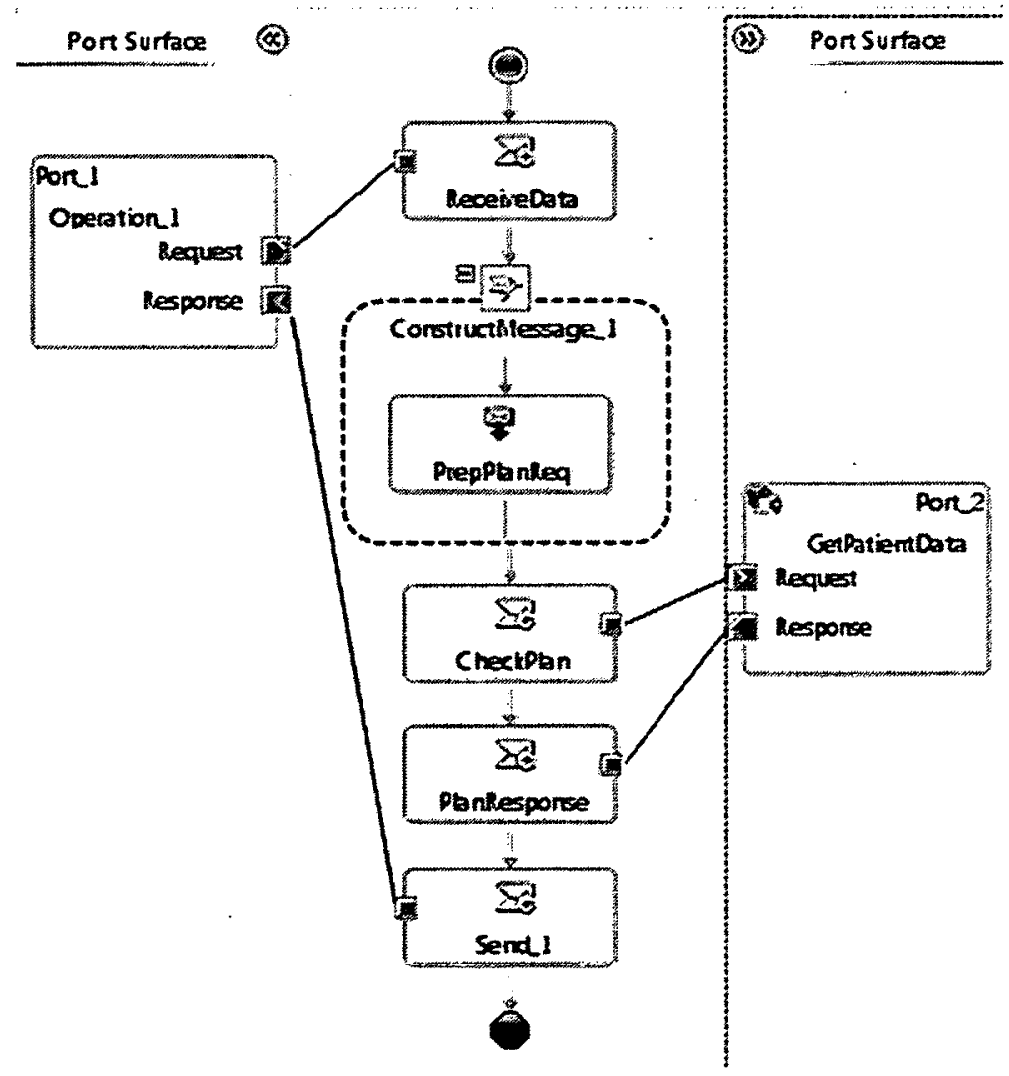

Figure 43 Implementation of insurance workflow in Biztalk 


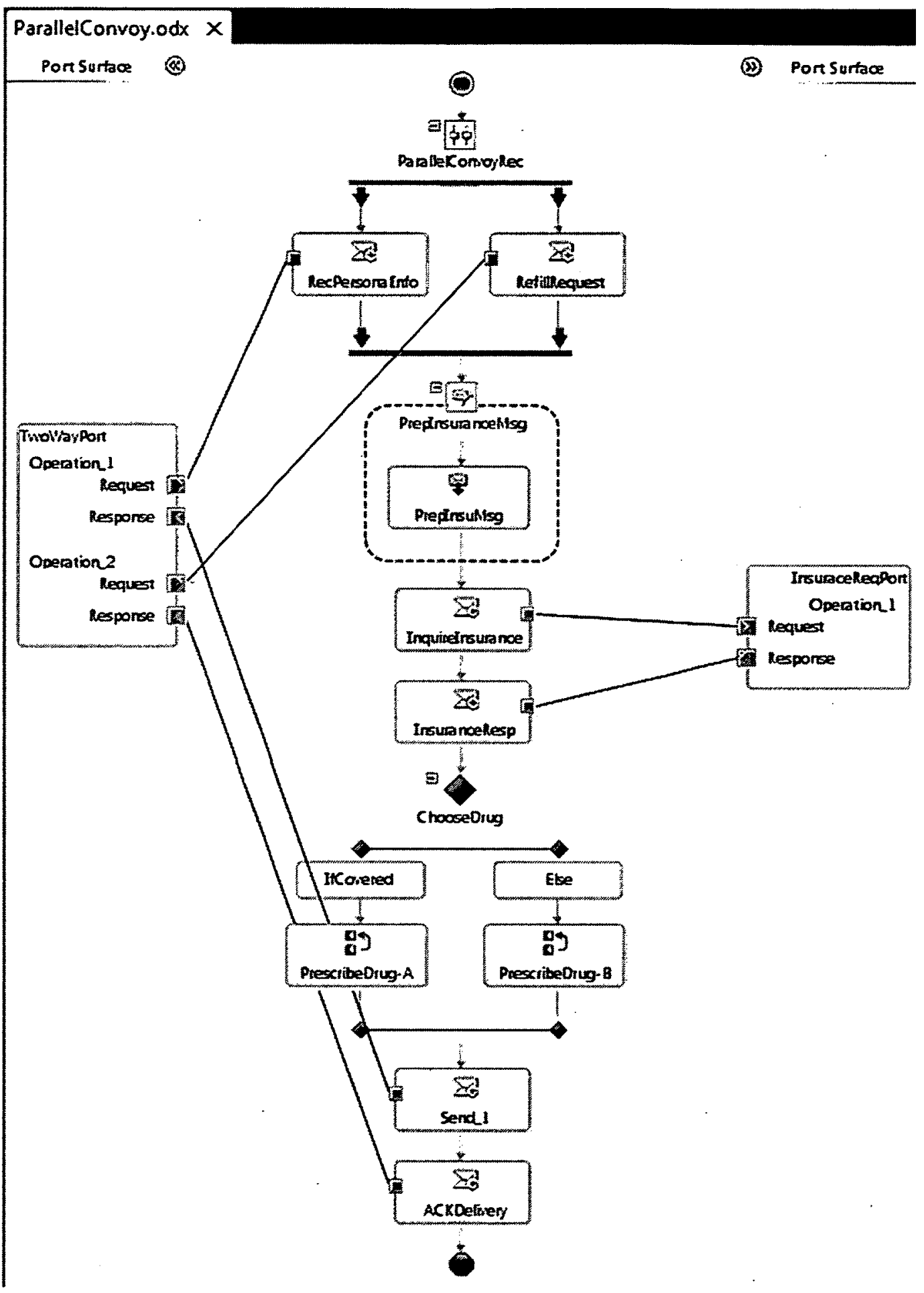

Figure 44 Designer implementation of prescription re-fill workflow in Biztalk 


\subsubsection{MEASUREMENTS}

We run the Biztalk implementation with SOAP UI and then automated the testing with LOAD-UI over a period of 60 minutes with 3 invocations per minute and the results are shown in Figure 45.

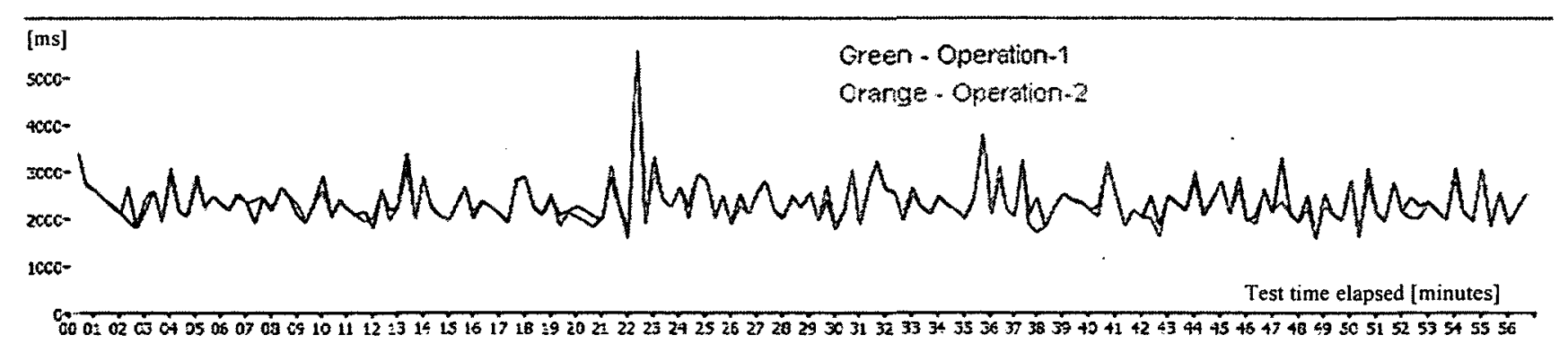

Figure 45 Patient prescription re-fill request workflow response times

Table 23 Average Response Time

\begin{tabular}{|l|c|}
\hline $\begin{array}{l}\text { Operation - Patient prescription request } \\
\text { workflow invoking the insurance checking } \\
\text { workflow }\end{array}$ & $\begin{array}{l}\text { Average Response } \\
\text { Time (ms) }\end{array}$ \\
\hline Operation - 1 & 2197.63 \\
\hline Operation -2 & 2136.23 \\
\hline
\end{tabular}

\subsubsection{CONFIDENCE INTERVAL OF MEASUREMENTS}

For the purpose of confidence interval calculation, we will break our 180 measurements for each operation into four batches, where each batch contains 45 values:

\begin{tabular}{|c|c|c|c|c|c|c|}
\hline Operation & $\begin{array}{c}\text { Batch1 } \\
\text { average }\end{array}$ & $\begin{array}{c}\text { Batch2 } \\
\text { average }\end{array}$ & $\begin{array}{c}\text { Batch3 } \\
\text { average }\end{array}$ & $\begin{array}{c}\text { Batch4 } \\
\text { average }\end{array}$ & Average & StdDev \\
\hline 1 & 2165.05 & 2271.00 & 2175.47 & 2179.00 & 2197.63 & 49.26 \\
\hline 2 & 2131.95 & 2125.25 & 2142.95 & 2144.78 & 2136.23 & 9.26 \\
\hline
\end{tabular}


For $95 \%$ confidence interval of 4 sample size, we use the following equation (where the coefficient 2.776 was taken from tables provided for t-Student distribution):

$$
\text { [Average-(StDev * 2.776), Average + }(S t D e v * 2.776)]
$$

For operation-1 we obtain the following confidence interval:

[2060.88 ms, $2334.37 \mathrm{~ms}$ ]

For operation-2 we obtain the following confidence interval:

[2110.52 ms, $2161.93 \mathrm{~ms}$ ]

We can observe that the actual value of the response time for each operation is rather close to the estimated value from section 5.3.1; in fact the relative error of the estimated value is within $15 \%$ of the actual values of both operations.

\subsection{ESTIMATION WITH VARIABLE MESSAGE SIZE}

In chapter 3, we performed measurements for variable message sizes; $1 \mathrm{~KB}$ had response time of $644 \mathrm{~ms}$, whereas that of $800 \mathrm{~KB}$ was $904 \mathrm{~ms}$, which is a difference of $260 \mathrm{~ms}$. If we assume that the prescription refill workflow requires $800 \mathrm{~KB}$ instead of $1 \mathrm{~KB}$, we can add an additional overhead of $260 \mathrm{~ms}$ on each operation due to the large message size. Therefore we can estimate that the responses time for each operation $800 \mathrm{~KB}$ will be:

Operation -1 Reponses Time $(1 \mathrm{~KB})+260=2197.63+260=2457 \mathrm{~ms}$

Operation -2 Response Time $(1 \mathrm{~KB})+260=2136+260=2396 \mathrm{~ms}$

We then actually tested the prescription refill workflow with $800 \mathrm{~KB}$ message size and obtained the results shown in the following figure: 


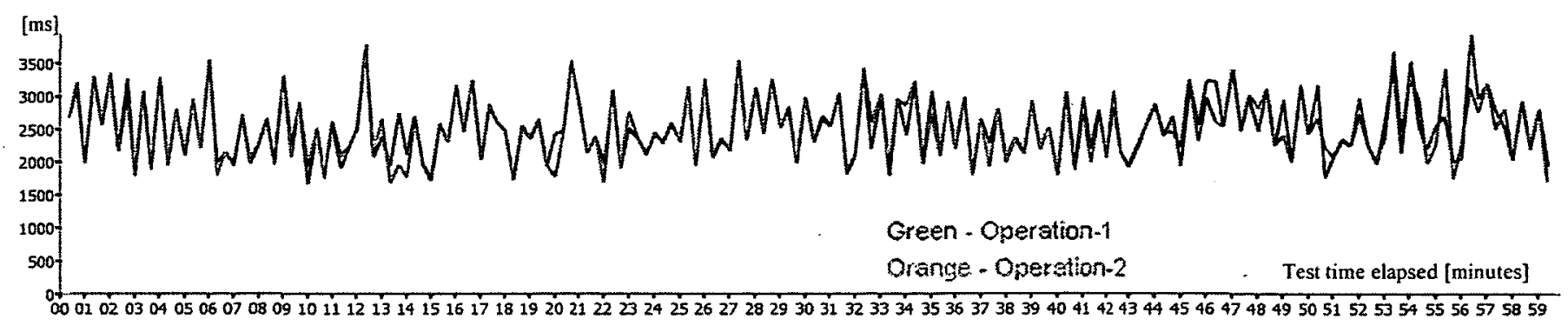

Figure 46 Response time for prescription refill workflow (with $800 \mathrm{~KB}$ messages)

Table 24 compares the average of response time of 180 invocations over 60 minutes with the above estimated values.

Table 24 Average response time comparison with estimated values $(800 \mathrm{~KB})$

\begin{tabular}{|c|c|c|}
\hline ms & Operation - 1 & Operation - \\
\hline Estimated (E) & 2457 & 2396 \\
\hline Actual (A) & 2530.21 & 2512.34 \\
\hline Difference (E-A) & -73.212 & -116.34 \\
\hline Relative Error (E-A)/A & $-2.89 \%$ & $-4.63 \%$ \\
\hline
\end{tabular}

Similarly following the same technique, the estimated response time for invoking the prescription refill workflow with $400 \mathrm{Kbyte}$ message size would be:

$$
\begin{gathered}
\text { Operation -1 Reponses Time }(1 \mathrm{~KB})+(790-644)=2197.63+146=2343.63 \mathrm{~ms} \\
\text { Operation-2 Response Time }(1 \mathrm{~KB})+(790-644)=2136+146=2282 \mathrm{~ms}
\end{gathered}
$$

The actual results are given in following figure:

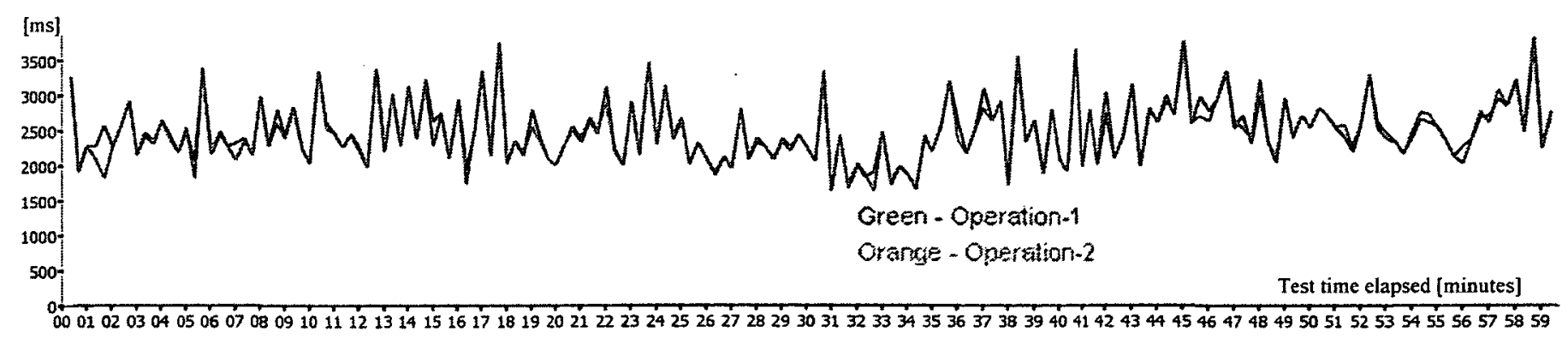

Figure 47 Response time for prescription refill workflow (with $400 \mathrm{~KB}$ messages) 
Table 25 compares the average of response time of 180 invocations over 60 minutes with the above estimated values.

Table 25 Average response time comparison with estimated values (400 KB)

\begin{tabular}{|c|c|c|}
\hline ms & Operation - & Operation -2 \\
\hline Estimated & 2343.63 & 2282 \\
\hline Actual & 2459.01 & 2436.75 \\
\hline Difference (E-A) & -115.38 & -154.75 \\
\hline Relative Error (E-A)/A & $-4.69 \%$ & $-6.35 \%$ \\
\hline
\end{tabular}

As a general observation, we realized that a significant performance overhead in the implementations of web services workflows is due to platform-provided patterns behind the scenes: publish-subscribe reliable messaging, de-hydration, re-hydration and correlation. This overhead stems from a variety of database operation, table look-ups and party resolution processes. Another important source of overhead is the amount of XML processing required in parsing and creation of XML messages. The designer should balance the advantages brought by the use of these patterns with the loss in performance and make appropriate design decisions early in the software lifecycle.

For instance, such considerations should be taken into account when deciding on the granularity of services and workflows exposed as services. Lower-granularity services may improve the reusability and coherence of the services in the system, but will increase the contribution of the platform overheads to the total response time. Early performance estimations will allow for such trade-offs to be made. 


\section{CONCLUSIONS}

In this work, we found that there are many patterns that are applied in SOA systems. Some patterns are applied at a very low level by the service platform, such as reliable messaging, message persistence and parsing of XML. Such patterns are applied behind the scene by the service platform to enhance the reliability of the application, but they introduce major performance overheads in the response time of the SOA applications. The developers have very little control over the application of these patterns, but it helps if they are aware of their performance costs. Therefore developers must exercise caution when deciding to use platform features that make use of these patterns, as in some cases, using such features may be overkill. The decision what to use and when should be made as a trade-off between performance and other software qualities (reliability and robustness in this case). Before deciding to opt for such a reliable system, proper analysis of requirements is necessary.

Other types of patterns, such as Scatter and Gather, Convoys and Content Based Router are explicitly applied by the developers when they build the application on top of a chosen platform. The developers have some flexibility to optimize their design to get some performance improvement, but there is a default overhead due to existence of platform patterns and their impact on performance is overwhelming. The choice of these

development level patterns should be influenced by a trade-off analysis between a particular business case and the need to improve performance. 
In real life implementations, there is a lot of emphasis on design, development and functional testing. Also due to time constraints there is not much interest in performance evaluation and enhancements until late, naturally due to non-availability of a functional system that can be measured. Usually there is more emphasis on performance evaluation when a system is built, after all the investment in time and money. Late changes undertaken to improve performance are very costly. Therefore our approach suggests the use quantitative performance analysis, which can be used to estimate the system performance before its actual implementation. This approach can address many performance problems early rather at later stages when it is very difficult to apply even minor modifications. In the thesis, we showed how knowledge about the quantitative overheads can be used to estimate the end-to-end response time of different workflows. A more advanced approach would be to build a performance model of the system, as recommended by the Software Performance Engineering methodology (Smith, 1990). Such performance models require quantitative parameters regarding the resource demands made by different workflow and services steps. Our work can help provide such quantitative values to performance modeler. For instance, our measurements can be used as input by other researchers that are concerned with building performance models for SOA systems, such as (Alhaj and Petriu, 2010), (Mani et al., 2012).

An important challenge for the thesis stems from the fact that the performance characteristics of a service-oriented system depends not only on the software application, but also on the underlying platforms: service execution engine, middleware, operating system, communication networks and hardware. The performance contributions of these 
elements are quite difficult to identify and separate, mainly due to the encapsulation of details inside the components and modules composing all these layers and the limitation of the measurement tools that are available.

With the use of right performance estimation techniques, more robust systems can be built which are prone to fewer modifications for performance enhancements. Also a careful analysis and knowledge of platform can help to better match the requirements of the system being built with the capabilities offered by the platform, thereby eliminating usage of any unnecessary patterns degrading the system performance.

\subsection{LIMITATIONS OF THE WORK}

The patterns list implemented in this work is not exhaustive. We have focused on messaging patterns because messaging is an important part of SOA systems and has important performance effects. There are several other patterns that are being implemented at platform level and others that can be utilized at development time: These patterns differ in scope and are dictated by other non-functional system requirements such as maintainability, reusability, secure communication.

During this work, all the implementations were performed locally on a single computer to eliminate the variance in response time due to network delays and availability. At the beginning of our research, we conducted some measurements on two system interconnected by a Local Area Network, but we realized that the variations in network communication delay due to the interference of other workload was hard to understand and explain. 
In a more realistic scenario, the processes presented in this work such as hospital service and insurance services are provided by different parties connected via public networks. Also the tests were implemented for one concurrent user request at a time, not considering multiple concurrent users and queuing delays, such as in other work in literature, such as (El Saddik, 2006). The main reason is that we did not find appropriate measurement tools that would allow us to separate the queueing delays from execution times of different software steps. Future work is necessary to overcome these limitations.

\subsection{FUTURE WORK}

This work represents a first step for performance measurements and evaluations of patterns applied in SOA applications. There are various dimensions for testing and analysis in the following foreseeable directions:

- Apply and measure other patterns that can be applied to more diverse business cases, not covered by the case studies in this work.

- Devise methods and find tools to measure resource demands and performance results at different granularity levels. For instance, we found no explicit support provided by testing and measurement tools to allow us to measure what happens at platform level.

- Perform measurements under multiple users and varying workload conditions.

- Perform measurements in a distributed network scenario where various services in an integration application are hosted on multiple computers on a local network. This will allow considering the effect of network delays. 
- Use the measurements in this work as input parameters to performance models for complete predictive performance analysis. 


\section{References}

Ajmone Marsan M., Balbo G., Conte G., Donatelli S. and Franceschinis G., (1995) Modelling with Generalized Stochastic Petri Nets, Wiley Series in Parallel Computing, John Wiley and Sons.

Adams, S., Hardas, D., Hossain, A., Cannon, C.R., Morimoto, R., Price, K.T., Tranchida, (2002) Biztalk Unleashed, Sams Publishing.

Alhaj, M., Petriu,D.C. (2010) "Approach for generating performance models from UML models of SOA systems", Proc. of CASCON 2010, Toronto, Nov. 2010.

Biztalk 2010, http://www.microsoft.com/biztalk/en/us/default.aspx, consulted 20 December, 2012

Biztalk Orchestration Profiler, www.biztalkorcprofiler.codeplex.com, consulted 20 December, 2012.

Buschmann, F., Meunier, R., Rohnert H., Sommerlad (1996). Pattern-Oriented Software Architecture, Volume 1: A System of Patterns. John Wiley \& Sons.

Barros, A., Dumas, M., ter Hofstede, A.H.M. (2005). Service Interaction Patterns. In Business Process Management, Springer, LNCS Volume 3649, pp.302-318.

Earl, T. (2005). Service-Oriented Architecture (SOA): Concepts, Technology, and Design, Pearson Ed. 
Earl, T. (2008). Introducing SOA Design Patterns, SOA World Magazine, Vol.8, Issue 6, pp. 2-7.

Earl, T. (2009). SOA Design Patterns. Prentice Hall ,Service-Oriented Computing Series

El Saddik, A. (2006). Performance Measurements of Web Services-Based Applications. IEEE Transactions on Instrumentation and Measurements, Vol. 55, No 5, pp 1599-1605.

Fielding, R. (2000). Architectural Styles and the Design of Network-based Software Architectures. Ph.D. Dissertation., University of California, Irvine.

Gamma, E., Helm, R., Johnson, R., Vlissides, J. (1994) Design Patterns: Elements of Reusable Object-Oriented Software. Addison-Wesley.

Hewitt, E. (2009). JAVA SOA cookbook, O'Reilly Media.

Hohpe, G., Woolf, B. (2003) Enterprise Integration Patterns. Addison-Wesley.

S., Kaiman, C., Havlioglu, C., Oikawa, R., Pearson, R., Lake, T., Larry Wall, L. (2002). Biz Talk Unleashed, Sams.

Lazowska E., Zahorjan J., Scott Graham G., Sevcik K.S., (1984) Quantitative System Performance: Computer System Analysis Using Queueing Network Models, Prentice Hall, 1984.

LoadUI, www.loadui.org, consulted 20 Dec 2012.

Khan, M.K., Petriu,D.C. (2012) "Performance Measurements of Design Patterns for Service- Oriented Architecture", Proc. of the International Conference on Electrical and Computer Engineering ICECS’2012, Ottawa ON, Canada, August 2012. 
Mani, N., D.C. Petriu, Woodside, C.M. (2011) "Studying the Impact of Design Patterns on the Performance Analysis of Service Oriented Architecture", Proc. of the 37th EuroMicro Conference on Software Engineering and Advanced Applications, Oulu, Finland, Sept. 2011

Partridge, C., Bassi, J. (2011). Electronic Prescribing Workflow Analysis Handbook v3.0.

Retrieved from ehealth.uvic.ca/resources/tools/WorkflowModeling/

WorkflowModeling.php

Rosanova, D. (2011). Microsoft BizTalk Server 2010 Patterns, PACKT Publishing.

SOA Patterns, www.soapatterns.org/, consulted 20 Dec 2012.

soapUI, www.soapui.org, consulted 20 Dec 2012.

Schumacher, M. and Roedig, U. (2001) Security engineering with patterns. In 8th Conference on Pattern Languages of Programs, July 2001.

Schmidt, D C., Stal, Rohnert, Buschmann (2000). Pattern-Oriented Software Architecture, Volume 2: Patterns for Concurrent and Networked Objects. John Wiley \& Sons.

Smith, C.U. (1990) Performance Engineering of Software Systems. Addison-Wesley.

Smith, C.U., Williams, L.G.(2002), Performance Solutions: A Practical Guide to Creating. Responsive, Scalable Software, Addison-Wesley. 
Woodside, J.E. Neilson, D.C. Petriu, S. Majumdar, (1995)"The Stochastic Rendezvous Network Model for Performance of Synchronous Client-Server-like Distributed Software," IEEE Transactions on Computers, Vol.44, No.1, pp. 20-34.

Yoder, J and J. Barcalow., J. (1997) Architectural patterns for enabling application security. Conference on Pattern Languages of Programs. PLoP '97. 